\title{
Bounded $H^{\infty}$-calculus for a degenerate elliptic boundary value problem
}

\author{
Thorben Krietenstein $^{1} \cdot$ Elmar Schrohe $^{1}$ (B)
}

Received: 9 September 2020 / Revised: 26 July 2021 / Accepted: 26 July 2021 /

Published online: 17 August 2021

(c) The Author(s) 2021

\begin{abstract}
On a manifold $X$ with boundary and bounded geometry we consider a strongly elliptic second order operator $A$ together with a degenerate boundary operator $T$ of the form $T=\varphi_{0} \gamma_{0}+\varphi_{1} \gamma_{1}$. Here $\gamma_{0}$ and $\gamma_{1}$ denote the evaluation of a function and its exterior normal derivative, respectively, at the boundary. We assume that $\varphi_{0}, \varphi_{1} \geq 0$, and $\varphi_{0}+\varphi_{1} \geq c$, for some $c>0$, where either $\varphi_{0}, \varphi_{1} \in C_{b}^{\infty}(\partial X)$ or $\varphi_{0}=1$ and $\varphi_{1}=\varphi^{2}$ for some $\varphi \in C^{2+\tau}(\partial X), \tau>0$. We also assume that the highest order coefficients of $A$ belong to $C^{\tau}(X)$ and the lower order coefficients are in $L_{\infty}(X)$. We show that the $L_{p}(X)$-realization of $A$ with respect to the boundary operator $T$ has a bounded $H^{\infty}$-calculus. We then obtain the unique solvability of the associated boundary value problem in adapted spaces. As an application, we show the short time existence of solutions to the porous medium equation.
\end{abstract}

Mathematics Subject Classification Primary 58J32; Secondary 35J25 • 35K20 . $58 \mathrm{~J} 35$

\section{Contents}

1 Introduction . . . . . . . . . . . . . . . . . . . . . . . . 1598

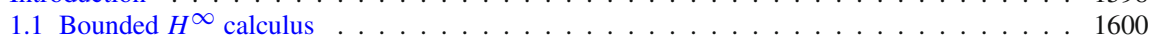

1.2 Main results . . . . . . . . . . . . . . . . . . . . . . . . . . . . . . . . . . . . . . . . . . . . . . . . . . . . . . . . . . . .

1.3 Relation to previous work . . . . . . . . . . . . . . . . . . . . . . 1602

1.4 Outline of the paper . . . . . . . . . . . . . . . . . . . . . . 1603

2 An extended Boutet de Monvel type calculus . . . . . . . . . . . . . . . . . . . . . 1605

Communicated by Y. Giga.

Elmar Schrohe

schrohe@math.uni-hannover.de

Thorben Krietenstein

krietenstein@math.uni-hannover.de

1 Institut für Analysis, Leibniz Universität Hannover, Welfengarten 1, 30167 Hannover, Germany 
2.1 Operator-valued symbols . . . . . . . . . . . . . . . . . . . . . . . . . . . . . . . . 1606

2.2 The transmission condition . . . . . . . . . . . . . . . . . . . . . . 1606

2.3 Potential, trace and singular Green symbols ． . . . . . . . . . . . . . . . . . . . . 1607

2.4 Boundary symbols and operators . . . . . . . . . . . . . . . . . . . . . . . . . . . . 1609

3 The resolvent . . . . . . . . . . . . . . . . . . . . . . . . . 1610

3.1 The dependence on the spectral parameter $\mu \ldots \ldots \ldots \ldots$. . . . . . . . . . . 1612

3.2 The principal symbol of the degenerate singular Green operator . . . . . . . . . . . . 1617

3.3 The parametrix on the boundary . . . . . . . . . . . . . . . . . . . 1618

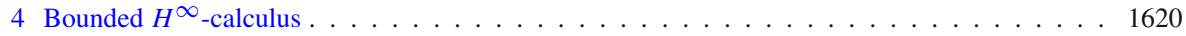

4.1 The half space and constant coefficients . . . . . . . . . . . . . . . . . 1620

4.2 The Euclidean half space . . . . . . . . . . . . . . . . . . . . . . . 1622

4.3 Manifolds . . . . . . . . . . . . . . . . . . . . . . . . . . . . . 1628

5 Non-smooth boundary operators . . . . . . . . . . . . . . . . . . . . . . . . . . . . 1629

6 The porous medium equation $\ldots \ldots \ldots$. . . . . . . . . . . . . . . . . . 1639

6.1 Quasi-linear evolution equations . . . . . . . . . . . . . . . . . . . . . . . . 1639

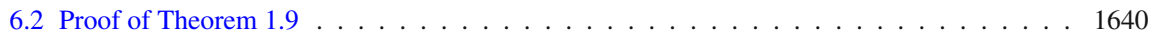

References . . . . . . . . . . . . . . . . . . . . . . . . . . . . . 1644

\section{Introduction}

Maximal regularity has become an indispensable tool in the analysis of evolution equations as it can be used to establish in an uncomplicated way the existence of short time solutions to a large class of quasilinear parabolic problems. Maximal regularity in turn is implied by the existence of a bounded $H^{\infty}$-calculus, a concept introduced by McIntosh in 1986, [29], of angle $<\pi / 2$. Many elliptic operators are known to have a bounded $H^{\infty}$-calculus, see e.g. Amann, Hieber, Simonett [5] for the case of differential operators. Already in 1971 Seeley [37] had shown that differential boundary value problems have bounded imaginary powers, a property which is very close to that of having a bounded $H^{\infty}$-calculus and can often be shown by the same methods. Ellipticity, however, is not necessary in this context as shown in [9]; a hypoellipticity condition in the spirit of Hörmander's conditions (4.2)' and (4.4)' in [22] is sufficient. In the present article, we establish the existence of a bounded $H^{\infty}$-calculus for a degenerate elliptic boundary value problem. We consider a strongly elliptic operator $A$, endowed with a boundary operator that, in general, will not satisfy the LopatinskyShapiro ellipticity condition. The key point of our analysis then is the construction of a parameter-dependent parametrix to the resolvent with the help of Boutet de Monvel's calculus for boundary value problems [8]. As a consequence of the non-ellipticity, however, this parametrix will only belong to an extended version of Boutet de Monvel's calculus that we sketch, below. Still, this will enable us to deduce the necessary estimates for the existence of the bounded $H^{\infty}$-calculus.

Here are the details. Let $X$ be an $n$-dimensional manifold with boundary $\partial X$ and bounded geometry. Let $A$ be a strongly elliptic second order partial differential operator on $X$ which in local coordinates can be written in the form

$$
A=\sum_{1 \leq k, l \leq n} a^{k l}(x) D_{k} D_{l}+\sum_{1 \leq k \leq n} b^{k}(x) D_{k}+c^{0}(x),
$$


where $a^{k l} \in C^{\tau}(X)$ are real-valued, ${ }^{1}$ the matrix $\left(a^{k l}(x)\right)_{1 \leq k, l \leq n}$ is positive definite with a uniform positive lower bound, $b^{k}, c^{0} \in L_{\infty}(X)$, and $D_{k}=-i \partial_{x_{k}}$. If these conditions are met, we call the operator $A$ sufficiently regular. Furthermore, we say that $A$ is $M$-elliptic, if all the norms of the coefficients are bounded by $M>0$ and the positive lower bound of the matrix is given by $1 / M$. Obviously, this is no restriction as every operator as above is $M$-elliptic for some $M$. The operator $A$ is endowed with a boundary operator $T$ of the form

$$
T=\varphi_{0} \gamma_{0}+\varphi_{1} \gamma_{1}
$$

Here $\gamma_{0}$ denotes the trace operator and $\gamma_{1}$ the exterior normal derivative at $\partial X$. Moreover, $\varphi_{0}, \varphi_{1} \in C_{b}^{\infty}(\partial X)$ are real-valued functions on the boundary with $\varphi_{0}, \varphi_{1} \geq 0$ and $\varphi_{0}+\varphi_{1} \geq c>0$. We obtain the classical Dirichlet problem for $\varphi_{0}=1, \varphi_{1}=0$. The choice $\varphi_{0}=0, \varphi_{1}=1$ yields Neumann boundary conditions, and Robin problems correspond to the case where $\varphi_{1}$ is nowhere zero.

For given functions $f$ and $\phi$ we consider the boundary value problem with spectral parameter $\lambda$

$$
(A-\lambda) u=f \text { in } X, \quad T u=\phi \text { on } \partial X,
$$

in $L_{p}(X), 1<p<\infty$. To this end we introduce the $L_{p}$-realization of the above boundary value problem, i.e. the unbounded operator $A_{T}$, acting like $A$ on the domain

$$
\mathcal{D}\left(A_{T}\right):=\left\{u \in L_{p}(X): A u \in L_{p}(X), T u=0 \text { on } \partial X\right\} .
$$

This problem has been investigated by many authors, see e.g. Egorov-Kondrat'ev [16], Kannai [24] or Taira [42,43,45], also for the case where the boundary operator $T$ involves an additional first order tangential differential operator. This makes the analysis more subtle and will be treated in a subsequent publication.

We recall the notion of sectoriality:

Definition 1.1 A closed and densely defined operator $B: \mathcal{D}(B) \subset E \rightarrow E$, acting in a Banach space $E$ that is injective with dense range is called sectorial of type $\omega<\pi$, if for every $\omega<\theta<\pi$ there exists a constant $C_{\theta}$, such that

$$
\sigma(B) \subset \Sigma_{\theta} \text { and }\left\|\lambda(B-\lambda)^{-1}\right\|_{\mathcal{L}(X)} \leq C_{\theta} \text { for all } \lambda \in \mathbb{C} \backslash \Sigma_{\theta} .
$$

Here $\Sigma_{\theta}=\{\lambda \in \mathbb{C} \backslash\{0\}:|\arg (\lambda)| \leq \theta\} \cup\{0\}$ is the sector of angle $\theta$ around the positive real axis.

It has been shown by Taira that, for a bounded domain $X$, the $L_{p}$-realization $A_{T}$ is sectorial of type $\varepsilon$ for every $\varepsilon>0$, possibly after replacing $A$ by $A+c$ for a positive constant $c$. In particular, it generates an analytic semigroup. For details see e.g. [43, Theorem 1.2].

\footnotetext{
1 See also Remark 1.10(b) for the complex case.
} 


\subsection{Bounded $H^{\infty}$ calculus}

By $H^{\infty}\left(\Sigma_{\theta}\right)$ we denote the space of bounded holomorphic functions in the interior of the sector $\Sigma_{\theta}$ and by $H_{*}^{\infty}\left(\Sigma_{\theta}\right)$ the subspace of all functions $f$ such that $|f(\lambda)| \leq$ $C\left(|\lambda|^{\epsilon}+|\lambda|^{-\varepsilon}\right)^{-1}$ for suitable $C, \varepsilon>0$. It is well-known that this is a dense subspace with respect to the topology of uniform convergence on compact sets.

For a sectorial operator $B$ of type $\left.\omega, \theta^{\prime} \in\right] \omega, \theta\left[\right.$ and $f \in H_{*}^{\infty}\left(\Lambda_{\theta}\right)$ let

$$
f(B)=\frac{i}{2 \pi} \int_{\partial \Lambda_{\theta^{\prime}}} f(\lambda)(B-\lambda)^{-1} d \lambda \in \mathcal{L}(E) .
$$

The integral exists due to the sectoriality and is independent of the choice of $\theta^{\prime}$ by Cauchy's integral theorem. Given $f \in H^{\infty}\left(\Sigma_{\theta}\right)$, we can approximate $f$ by a sequence $\left(f_{n}\right) \subset H_{*}^{\infty}\left(\Sigma_{\theta}\right)$ and define

$$
f(B) x:=\lim f_{n}(B) x \text { for } x \in \mathcal{D}(B) \cap \operatorname{range}(B) .
$$

It can be shown that $\mathcal{D}(B) \cap \operatorname{range}(B)$ is dense in $E$ and that the above equation defines a closable operator. The closure is again denoted by $f(B)$.

Definition 1.2 We say that a sectorial operator $B$ of type $\omega$ admits a bounded $H^{\infty}$ calculus of angle $\omega$, if for any $\omega<\theta<\pi$ there exists a constant $C_{\theta}>0$, such that

$$
\|f(B)\|_{\mathcal{L}(E)} \leq C_{\theta}\|f\|_{\infty}, \quad f \in H^{\infty}\left(\Sigma_{\theta}\right)
$$

According to the principle of uniform boundedness it is sufficient to verify estimate (1.3) for all $f \in H_{*}^{\infty}\left(\Sigma_{\theta}\right)$.

\subsection{Main results}

Theorem 1.3 Let $(X, g)$ be a manifold with boundary and bounded geometry. Let $T$ be as in (1.2) and $A_{T}$ be the realization given above of an $M$-elliptic sufficiently regular second order differential operator. Then, for every $0<\vartheta<\pi$ a constant $v=v\left(M,|t|_{*}, \vartheta\right) \geq 0$ exists such that $A_{T}+v$ allows an $H^{\infty}\left(\Sigma_{\vartheta}\right)$-calculus in $L_{p}(X)$. Moreover, a constant $C=C\left(M,|t|_{*}, \vartheta\right)>0$ exists such that for all $f \in H^{\infty}\left(\Sigma_{\vartheta}\right)$ the following estimate holds:

$$
\left\|f\left(A_{T}\right)\right\|_{\mathcal{B}\left(L_{p}(X)\right)} \leq C\|f\|_{\infty}
$$

Here $|t|_{*}$ stands for suitable seminorms of $\varphi_{0}$ and $\varphi_{1}$, respectively, in $C_{b}^{\infty}(\partial X)$. Theorem 1.3 extends to the case of non-smooth boundary operators. In order to keep the technical difficulties at a reasonable level, we shall then assume that $\varphi_{0}=1$ and $\varphi_{1}$ is the square of a $C^{2+\tau}$-function $\varphi$ with $\tau>0$ :

$$
T=\gamma_{0}+\varphi^{2} \gamma_{1}
$$


for some $\varphi \in C^{2+\tau}, \tau>0$. Supposing that $\varphi_{0}=1$ is not an essential restriction. The assumption that $\varphi_{1}$ is the square of a $C^{2+\tau}$-function is; it is motivated by the fact that the pseudodifferential techniques require the $C^{2+\tau}$ regularity of the square root of $\varphi_{1}$, which differs from the regularity of $\varphi_{1}$ near the zero set. Here is the precise statement.

Theorem 1.4 Let $(X, g)$ and $A$ be as in Theorem 1.3 and $T$ as in (1.4). For every $0<\vartheta<\pi$ we then find $v=v\left(M,|t|_{*}, \vartheta\right) \geq 0$ and $C=C\left(M,|t|_{*}, \vartheta\right)>0$ such that $A_{T}+v$ allows an $H^{\infty}\left(\Sigma_{\vartheta}\right)$-calculus in $L_{p}(X)$ and

$$
\left\|f\left(A_{T}\right)\right\|_{\mathcal{B}\left(L_{p}(X)\right)} \leq C\|f\|_{\infty}, \quad f \in H^{\infty}\left(\Sigma_{\vartheta}\right) .
$$

As a corollary, we obtain unique solvability for the full boundary value problem. For this we need some notation. As before, $(X, g)$ is a manifold with boundary and bounded geometry, $1<p<\infty$.

We denote by $B_{p}^{s}(\partial X):=B_{p, p}^{s}(\partial X)$ the $L_{p}$-Besov space of order $s \in \mathbb{R}$ on $\partial X$ as defined in [17]. According to [17, Theorem 4.10], $B_{p}^{s-1 / p}(\partial X), 1<p<\infty$, $s>1 / p$, coincides with the space of all restrictions to $\partial X$ of functions in $H_{p}^{s}(X)$. The theorem, below, can be shown by modifying the proof of [17, Theorem 4.10] in the spirit of the proof of [47, Theorem 2.9.2].

Theorem 1.5 Let $s>1+1 / p$. Then, given $v_{0} \in B_{p}^{s-1 / p}(\partial X)$ and $v_{1} \in B_{p}^{s-1-1 / p}(\partial X)$ there exists $u \in H_{p}^{s}(X)$ such that $\gamma_{0} u=v_{0}$ and $\gamma_{1} u=v_{1}$.

Definition 1.6 For $s \in \mathbb{R}$ and the boundary condition $T$ in (1.2) or $s>-\tau$ and $T$ in (1.4), we define

$$
B_{p, T}^{s-1-1 / p}(\partial X)=\left\{v=\varphi_{0} v_{0}+\varphi_{1} v_{1} \mid v_{0} \in B_{p}^{s-1 / p}(\partial X), v_{1} \in B_{p}^{s-1-1 / p}(\partial X)\right\} .
$$

Clearly, this is a Banach space with the topology of the non-direct sum.

Proposition 1.7 For $s>1+1 / p$ the mapping $T: H_{p}^{s}(X) \rightarrow B_{p, T}^{s-1-1 / p}(\partial X)$ is surjective.

In fact, given $v=\varphi_{0} v_{0}+\varphi_{1} v_{1}$ in $B_{p, T}^{s-1-1 / p}(\partial X)$, Theorem 1.5 implies that we find $u_{0}$ and $u_{1}$ in $H_{p}^{s}(X)$ such that $\gamma_{0} u_{0} \stackrel{p, T}{=} v_{0}, \gamma_{1} u_{0}=0, \gamma_{0} u_{1}=0$ and $\gamma_{1} u_{1}=v_{1}$. Then $u_{0}+u_{1}$ is a preimage of $v$ under $T$.

Theorem 1.8 For every $0<\vartheta<\pi$ the operator

$$
\left(\begin{array}{c}
A-\lambda \\
T
\end{array}\right): H_{p}^{2}(X) \longrightarrow \underset{B_{p, T}^{1-1 / p}(\partial X)}{\bigoplus}
$$

is a topological isomorphism for $\lambda \in \Sigma_{\vartheta},|\lambda|$ sufficiently large.

This is immediate from Theorem 1.3 and the surjectivity of $T$ : Given $f \in L_{p}(X)$ and $v \in B_{p, T}^{1-1 / p}(\partial X)$, we first fix $w_{0} \in H_{p}^{2}(X)$ with $T w_{0}=v$. By Theorem 1.3, the 
problem $(A-\lambda) w=f-(A-\lambda) w_{0}, T u=0$ has a unique solution $w \in H_{p}^{2}(X)$. Then $u=w+w_{0}$ is the (unique) solution to $(A-\lambda) u=f, T u=v$. Hence (1.5) is a bijection. As it is continuous, it is a topological isomorphism in view of the closed graph theorem.

Finally, we apply our results to the porous medium equation with boundary condition $T$ and strictly positive initial value. Details can be found in Sect. 6. We obtain:

Theorem 1.9 Let $1<p, q<\infty, n / p+2 / q<1, m>0, v_{0} \in H_{p}^{2}(X)$ with $v_{0} \geq c>0$, and $\phi \in C^{1}\left(J_{0} ; B_{p, T}^{1-1 / p}(\partial X)\right)$ with $\phi(0)=T v_{0}$. Here $J_{0}=\left[0, t_{0}\right]$ with $t_{0}>0$. Then the porous medium equation

$$
\left\{\begin{array}{l}
\dot{v}-\Delta_{g} v^{m}=0 \\
T v=\phi \\
\left.v\right|_{t=0}=v_{0}
\end{array}\right.
$$

has a unique short time solution of maximal regularity, i.e. there exists an interval $J=\left[0, t^{*}\right]$ with $t^{*}>0$ and a unique solution

$$
v \in L_{q}\left(J ; H_{p}^{2}(X)\right) \cap W_{q}^{1}\left(J ; L_{p}(X)\right)
$$

of the porous medium Eq. (1.6).

Remark 1.10 (a) If the boundary $\partial X$ is the disjoint union of two open subsets $Y_{0}$ and $Y_{1}$ and their common boundary $\Sigma$, which additionally is a smooth embedded submanifold of $\partial X$, and we choose $\varphi_{0}$ and $\varphi_{1}$ to be the characteristic functions of $Y_{0}$ and $Y_{1}$, we obtain the Zaremba problem, where Dirichlet conditions are imposed on $Y_{0}$ and Neumann conditions on $Y_{1}$.

In this sense, the Zaremba problem might be considered a limit case of the degenerate problem studied here. However, the two problems are rather different in spirit. As our arguments show, the problem here can still be treated by methods from the theory of boundary value problems via Boutet de Monvel's calculus. The Zaremba problem in contrast is basically an edge-degenerate problem, as explained by Seeley in [38]. The role of the edge is played by the interface $\Sigma$, and additional conditions have to be imposed there. A corresponding singular pseudodifferential calculus has been developed by Dines, Harutyunyan and Schulze, see [14].

(b) For convenience we have assumed that the coefficients of $A$ are real. In fact, the results also hold in the parameter-elliptic complex-valued case with the corresponding change of the sector.

\subsection{Relation to previous work}

In [1], Abels developed a (different) variant of Boutet de Monvel's calculus with nonsmooth symbols in order to construct parametrices to elliptic operators with Hölder 
regularity. Also, Krainer [25] constructed a calculus with symbols of type $\rho, \delta$ taking values in operator ideals in Hilbert spaces.

Theorem 1.3 and 1.4 extend [43, Theorem 1.2] in that (i) one can now treat manifolds of bounded geometry instead of bounded domains, (ii) the differentiability assumptions on the coefficients of $A$ are reduced from $C^{\infty}$ to $C^{\tau}, \tau>0$, for the top order terms and $L_{\infty}$ for the lower order terms, while $T$ can also be of the form (1.4), and (iii) one obtains the existence of a bounded $H^{\infty}$-calculus rather than the existence of a holomorphic semigroup. Theorem 1.8 extends [43, Theorem 1.1] to the case of manifolds with boundary and bounded geometry and operators with non-smooth coefficients, with the restriction that $\lambda \in \Sigma_{\vartheta}$ has to be large and we work on $H_{p}^{2}(X)$ as a consequence of the non-smoothness of the coefficients.

In [44] and [41] Taira treats more general Waldenfels integro-differential operators to which the present methods should also be applicable.

While there is a wealth of literature on the porous medium equation, it seems to be new to study it on manifolds of bounded geometry and with degenerate boundary condition.

\subsection{Outline of the paper}

We first focus on the boundary operator $T$ in (1.2); the non-smooth case in (1.4) will be treated in Sect. 5 .

In order to establish (1.3) for $A_{T}+c$ we have to show that for every fixed $0<\theta<\pi$

$$
\left\|\int_{\partial \Lambda_{\theta}} f(\lambda)\left(A_{T}+c-\lambda\right)^{-1} d \lambda\right\|_{\mathcal{L}\left(L_{p}(X)\right)} \leq C_{\theta}\|f\|_{\infty}, \quad f \in H_{*}^{\infty}\left(\Lambda_{\theta}\right) .
$$

It is clear that a good understanding of $\left(A_{T}+c-\lambda\right)^{-1}$ on the rays $\arg \lambda= \pm \theta$, $0<\theta<\pi$ is essential for this task.

The main tool we use in this paper is Boutet de Monvel's calculus for boundary value problems [8]. Details can be found e.g. in the monographs by Rempel and Schulze [32] and Grubb [19] or in the short introduction [35]. We will also need a slight generalization for which details will be given below. Recall that an operator of order $m \in \mathbb{R}$ and class (or type) $d \in \mathbb{N}_{0}$ in Boutet de Monvel's calculus on $\mathbb{R}_{+}^{n}$ is a matrix of operators

$$
\left(\begin{array}{cc}
P_{+}+G & K \\
T & S
\end{array}\right): \underset{\mathcal{S}\left(\mathbb{R}^{n-1}, F_{0}\right)}{\oplus} \rightarrow \underset{\mathcal{S}\left(\mathbb{R}^{n-1}, F_{1}\right)}{\oplus}
$$

Here $E_{0}$ and $E_{1}$ are vector bundles over $\mathbb{R}^{n}$, and $F_{0}, F_{1}$ are vector bundles over $\partial \mathbb{R}_{+}^{n}=\mathbb{R}^{n-1}$. Moreover, $P$ is a pseudodifferential operator on $\mathbb{R}^{n}$ satisfying the transmission condition, and $P_{+}$denotes its truncation to $\mathbb{R}_{+}^{n}: P_{+}=r^{+} P e^{+}$, where $e^{+}$ denotes extension by zero from $\mathcal{S}\left(\mathbb{R}_{+}^{n}, E_{0}\right)$ to, say, $L_{2}\left(\mathbb{R}^{n}, E_{0}\right)$, and $r^{+}$denotes the restriction of distributions on $\mathbb{R}^{n}$ to those on $\mathbb{R}_{+}^{n}$. The operators $G$ and $T$ are singular Green and trace operators of order $m$ and class $d$, respectively; $K$ is a potential operator 
of order $m$. Finally $S$ is a pseudodifferential operator on the boundary $\mathbb{R}^{n-1}$ of order $m$.

Boutet de Monvel's calculus is closed under compositions provided the vector bundles fit together. Via coordinate maps the calculus can be transferred to smooth manifolds with boundary.

Boutet de Monvel's calculus has a symbolic structure with a notion of ellipticity, and there exist parametrices to elliptic elements in the calculus. Moreover, the calculus contains its inverses whenever these exist. An operator of order $m$ and class $d$ as above extends to a bounded map

$$
H_{p}^{s+m}\left(X, E_{0}\right) \oplus B_{p}^{s+m-1 / p}\left(\partial X, F_{0}\right) \rightarrow H_{p}^{s}\left(X, E_{1}\right) \oplus B_{p}^{s-1 / p}\left(\partial X, F_{1}\right),
$$

provided $s>d-1+1 / p$, where $H_{p}^{s}$ denotes the usual Sobolev space and $B_{p}^{s}=B_{p, p}^{s}$ the Besov space of order $s$, see Grubb [18].

It is well-known that the operator

$$
\left(\begin{array}{c}
(A-\lambda)_{+} \\
\gamma_{0}
\end{array}\right): H_{p}^{2}(X) \rightarrow \underset{B_{p}^{2-1 / p}(\partial X)}{\bigoplus}
$$

is invertible for $\lambda \in \Lambda_{\theta}, \theta>0,|\lambda|$ sufficiently large, whenever $X$ is a compact manifold with boundary or $\mathbb{R}_{+}^{n}$. In particular, it is invertible for all $\lambda \in \Lambda_{\theta}$, if we replace $A$ by $A+c$ for $c>0$ sufficiently large. In order to keep the notation simple, we will assume from now on that $A$ has been replaced by $A+c$ for such $c$ and write $A$ instead of $A+c$.

Apart from the fact that $\gamma_{0}$ is formally not of the right order (which is of no importance here and can be easily arranged), the problem fits into Boutet de Monvel's calculus and one obtains the inverse in the form

$$
\left(\begin{array}{c}
(A-\lambda)_{+} \\
\gamma_{0}
\end{array}\right)^{-1}=\left(\left((A-\lambda)^{-1}\right)_{+}+G_{\lambda}^{D} \quad K_{\lambda}^{D}\right) .
$$

Here $(A-\lambda)^{-1}$ is the resolvent on a closed manifold with boundary into which $X$ embeds (in case $X$ is compact) or on $\mathbb{R}^{n}$ (if $X=\mathbb{R}_{+}^{n}$ ), see [19].

We will denote the corresponding truncation by $Q_{\lambda,+}$ :

$$
Q_{\lambda,+}=\left((A-\lambda)^{-1}\right)_{+}
$$

As a consequence,

$$
\left(\begin{array}{c}
(A-\lambda)_{+} \\
T
\end{array}\right)\left(Q_{\lambda,+}+G_{\lambda}^{D} \quad K_{\lambda}^{D}\right)=\left(\begin{array}{cc}
I & 0 \\
T\left(Q_{\lambda,+}+G_{\lambda}^{D}\right) T K_{\lambda}^{D}
\end{array}\right) .
$$


Assuming that $S_{\lambda}:=T K_{\lambda}^{D}$ is invertible with inverse $S_{\lambda}^{-1}$, we find that

$$
\begin{aligned}
& \left(\begin{array}{c}
(A-\lambda)_{+} \\
T
\end{array}\right)^{-1} \\
& =\left(Q_{\lambda,+}+G_{\lambda}^{D}-K_{\lambda}^{D} S_{\lambda}^{-1} T\left(Q_{\lambda,+}+G_{\lambda}^{D}\right) K_{\lambda}^{D} S_{\lambda}^{-1}\right) .
\end{aligned}
$$

For the realization $(A-\lambda)_{T}$ we obtain:

$$
\begin{aligned}
& (A-\lambda)_{T}^{-1} \\
& \quad=Q_{\lambda,+}+G_{\lambda}^{D}-K_{\lambda}^{D} S_{\lambda}^{-1} T\left(Q_{\lambda,+}+G_{\lambda}^{D}\right) \\
& \quad=Q_{\lambda,+}+G_{\lambda}^{D}+G_{\lambda}^{T}
\end{aligned}
$$

with

$$
G_{\lambda}^{T}:=-K_{\lambda}^{D} S_{\lambda}^{-1} T\left(Q_{\lambda,+}+G_{\lambda}^{D}\right)
$$

Lemma 1.11 For every choice of $\theta \in] 0, \pi\left[\right.$, there exists a constant $C_{\theta} \geq 0$ such that

$$
\left\|\int_{\partial \Lambda_{\theta}} f(\lambda)\left(Q_{\lambda,+}+G_{\lambda}^{D}\right) d \lambda\right\|_{\mathcal{L}\left(L_{p}(X)\right)} \leq C_{\theta}\|f\|_{\infty} \text { for all } f \in H_{*}^{\infty}\left(\Lambda_{\theta}\right) .
$$

Lemma 1.11 is well-known, the proof relies on the fact that the operators $Q_{\lambda}$ and $G_{\lambda}^{D}$ are parameter-dependent operators of order -2 in Boutet de Monvel's calculus, if one writes $-\lambda=\mu^{2} e^{i \theta}$ and considers $Q_{\lambda}$ and $G_{\lambda}^{D}$ as functions of $\mu$, see e.g. Grubb [19]. For the more general situation of a manifold with boundary and conic singularities, see [11].

It remains to study the term $G_{\lambda}^{T}$. It will turn out that $T K_{\lambda}^{D}$ is a hypoelliptic pseudodifferential operator of order 1 on the boundary. As we will see, it has a parametrix with local symbols in the Hörmander class $S_{1,1 / 2}^{0}$ which then agrees with $S_{\lambda}^{-1}$ up to a regularizing operator. In order to treat the composition of $S_{\lambda}^{-1}$ with the operators $K_{\lambda}^{D}$ and $Q_{\lambda,+}+G_{\lambda}^{D}$, we will need an extension of the classical Boutet de Monvel calculus.

\section{An extended Boutet de Monvel type calculus}

We recall the algebra $\mathcal{H}$ of functions on $\mathbb{R}:$ It is the direct sum

$$
\mathcal{H}=\mathcal{H}^{+} \oplus \mathcal{H}_{-1}^{-} \oplus \mathcal{H}^{\prime}
$$

where

$$
\mathcal{H}^{+}:=\left\{\mathcal{F}\left(e^{+} u\right): u \in \mathcal{S}\left(\mathbb{R}_{+}\right)\right\}, \quad \mathcal{H}_{-1}^{-}:=\left\{\mathcal{F}\left(e^{-} u\right): u \in \mathcal{S}\left(\mathbb{R}_{-}\right)\right\}
$$


and $\mathcal{H}^{\prime}$ is the space of all polynomials on $\mathbb{R}$. The sum is direct, since the functions in $\mathcal{H}^{+}$and $\mathcal{H}_{-1}^{-}$decay to first order.

It will be helpful to use also weighted Sobolev spaces on $\mathbb{R}_{+}$: For $\mathbf{s}=\left(s_{1}, s_{2}\right) \in \mathbb{R}^{2}$ we let $H_{p}^{\mathbf{s}}\left(\mathbb{R}_{+}\right)$denote the space of all $u \in \mathcal{D}^{\prime}\left(\mathbb{R}_{+}\right)$such that $\langle x\rangle^{s_{2}} u$ belongs to the ordinary Sobolev space $H_{p}^{s_{1}}\left(\mathbb{R}_{+}\right)$. We then have

$$
\begin{aligned}
\mathcal{S}\left(\mathbb{R}_{+}\right) & =\operatorname{proj}-\lim H_{p}^{\mathbf{s}}\left(\mathbb{R}_{+}\right) \text {and } \\
\mathcal{S}^{\prime}\left(\mathbb{R}_{+}\right) & =\operatorname{ind}-\lim \left(H_{p}^{\mathbf{s}}\left(\mathbb{R}_{+}\right)\right)^{\prime}=\operatorname{ind}-\lim \dot{H}_{1-1 / p}^{\mathbf{s}}\left(\mathbb{R}_{+}\right),
\end{aligned}
$$

where the limits are taken over $\mathbf{s} \in \mathbb{R}^{2}$ and $\dot{H}_{q}^{\mathbf{s}}\left(\mathbb{R}_{+}\right)$denotes all distributions $u$ in $H_{q}^{\mathbf{s}}(\mathbb{R})$ for which supp $u \subset \overline{\mathbb{R}}_{+}$.

\subsection{Operator-valued symbols}

Let $E, F$ be Banach spaces with strongly continuous group actions $\kappa_{\lambda}^{E}, \kappa_{\lambda}^{F}, \lambda>0$, as introduced by Schulze in [36]. Given $q \in \mathbb{N}, m \in \mathbb{R}, 0 \leq \delta<1$, we call a function $a=$ $a(y, \eta) \in C^{\infty}\left(\mathbb{R}^{q} \times \mathbb{R}^{q}, \mathcal{L}(E, F)\right)$ an operator-valued symbol in $S_{1, \delta}^{m}\left(\mathbb{R}^{q} \times \mathbb{R}^{q} ; E, F\right)$ if, for all multi-indices $\alpha, \beta$, there exist constants $C_{\alpha, \beta}$ such that

$$
\left\|\kappa_{\langle\eta\rangle^{-1}}^{F} D_{\eta}^{\alpha} D_{y}^{\beta} a(y, \eta) \kappa_{\langle\eta\rangle}^{E}\right\|_{\mathcal{L}(E, F)} \leq C_{\alpha, \beta}\langle\eta\rangle^{m-|\alpha|+\delta|\beta|} .
$$

In the sequel, we will mostly have the case where and $E$ and $F$ are either $\mathbb{C}$ or (weighted) Sobolev spaces over $\mathbb{R}$ or $\mathbb{R}_{+}$. On $\mathbb{C}$ we will use the trivial group action; on the Sobolev spaces we will use the action given by $\kappa_{\lambda} u(t)=\sqrt{\lambda} f(\lambda t)$. Note that this group action is unitary on $L^{2}(\mathbb{R})$ and $L^{2}\left(\mathbb{R}_{+}\right)$. Using the representations (2.1) and (2.2), the above definition extends to the case, where $E=\mathcal{S}\left(\mathbb{R}_{+}\right), E=\mathcal{S}^{\prime}\left(\mathbb{R}_{+}\right)$or $F=\mathcal{S}\left(\mathbb{R}_{+}\right)$, see [35] for details.

\subsection{The transmission condition}

Definition 2.1 A symbol $p \in S_{1, \delta}^{m}\left(\mathbb{R}^{n} \times \mathbb{R}^{n}\right)$ satisfies the transmission condition at $x_{n}=0$ provided that, for all $k \in \mathbb{N}_{0}$

$$
p_{[k]}\left(x^{\prime}, \xi^{\prime}, \xi_{n}\right):=\left(\partial_{x_{n}}^{k} p\right)\left(x^{\prime}, 0, \xi^{\prime},\left\langle\xi^{\prime}\right\rangle \xi_{n}\right) \in S_{1, \delta}^{m+\delta k}\left(\mathbb{R}^{n-1} \times \mathbb{R}^{n-1}\right) \hat{\otimes} \mathcal{H}
$$

We write $p \in \mathcal{P}_{1, \delta}^{m}\left(\mathbb{R}^{n-1} \times \mathbb{R}^{n-1}\right)$.

Remark 2.2 $\mathcal{P}_{1, \delta}^{\infty}:=\bigcup_{m} \mathcal{P}_{1, \delta}^{m}$ is closed under the usual symbol operations, i.e. addition, pointwise multiplication and inversion, differentiation, Leibniz product and asymptotic summation. We also have $S^{-\infty}=\bigcap_{m} \mathcal{P}_{1, \delta}^{m}$.

Theorem 2.3 Let $p \in \mathcal{P}_{1, \delta}^{m}\left(\mathbb{R}^{n} \times \mathbb{R}^{n}\right)$. Then

$$
\mathrm{op}_{n}(p)_{+} \in S_{1, \delta}^{m}\left(\mathbb{R}^{n-1} \times \mathbb{R}^{n-1} ; \mathcal{S}\left(\mathbb{R}_{+}\right), \mathcal{S}\left(\mathbb{R}_{+}\right)\right)
$$


Proof This follows from the fact that $\kappa_{\left\langle\xi^{\prime}\right\rangle^{-1}} \mathrm{op}_{n}(p) \kappa_{\left\langle\xi^{\prime}\right\rangle}=\mathrm{op}_{n}\left(q_{x^{\prime}, \xi^{\prime}}\right)$, where $q_{x^{\prime}, \xi^{\prime}}\left(x_{n}, \xi_{n}\right)=p\left(x^{\prime}, x_{n} /\left\langle\xi^{\prime}\right\rangle, \xi^{\prime},\left\langle\xi^{\prime}\right\rangle \xi_{n}\right)$ and the corresponding proof for Hörmander type $(1,0)$; this is Theorem 2.12 in [35]. The arguments carry over to general $(1, \delta)$.

\subsection{Potential, trace and singular Green symbols}

Definition 2.4 Let $m \in \mathbb{R}, d \in \mathbb{N}_{0}$. All functions, below, may be matrix valued.

- A function $k \in C^{\infty}\left(\mathbb{R}^{n-1} \times \mathbb{R}^{n-1} \times \mathbb{R}\right)$ belongs to the space $\mathcal{K}_{1, \delta}^{m}\left(\mathbb{R}^{n-1} \times \mathbb{R}^{n-1}\right)$ of potential symbols of order $m$ and Hörmander type $(1, \delta)$, if

$$
k_{[0]}\left(x^{\prime}, \xi^{\prime} ; \xi_{n}\right):=k\left(x^{\prime}, \xi^{\prime} ;\left\langle\xi^{\prime}\right\rangle \xi_{n}\right) \in S_{1, \delta}^{m-1}\left(\mathbb{R}^{n-1} \times \mathbb{R}^{n-1}\right) \hat{\otimes} \mathcal{H}_{\xi_{n}}^{+}
$$

- A function $t \in C^{\infty}\left(\mathbb{R}^{n-1} \times \mathbb{R}^{n-1} \times \mathbb{R}\right)$ belongs to the space $\mathcal{T}_{1, \delta}^{m, d}\left(\mathbb{R}^{n-1} \times \mathbb{R}^{n-1}\right)$ of trace symbols of order $m$, class $d$ and Hörmander type $(1, \delta)$, if

$$
t_{[0]}\left(x^{\prime}, \xi^{\prime} ; \xi_{n}\right):=t\left(x^{\prime}, \xi^{\prime} ;\left\langle\xi^{\prime}\right\rangle \xi_{n}\right) \in S_{1, \delta}^{m}\left(\mathbb{R}^{n-1} \times \mathbb{R}^{n-1}\right) \hat{\otimes} \mathcal{H}_{d-1}^{-} .
$$

- A function $g \in C^{\infty}\left(\mathbb{R}^{n-1} \times \mathbb{R}^{n-1} \times \mathbb{R} \times \mathbb{R}\right)$ belongs to the space $\mathcal{G}_{1, \delta}^{m, d}\left(\mathbb{R}^{n-1} \times\right.$ $\mathbb{R}^{n-1}$ ) of singular Green symbols of order $m$, class $d$ and Hörmander type $(1, \delta)$, if

$$
\begin{aligned}
& g_{[0]}\left(x^{\prime}, \xi^{\prime} ; \xi_{n}, \eta_{n}\right):=g\left(x^{\prime}, \xi^{\prime} ;\left\langle\xi^{\prime}\right\rangle \xi_{n},\left\langle\xi^{\prime}\right\rangle \eta_{n}\right) \\
& \in S_{1, \delta}^{m-1}\left(\mathbb{R}^{n-1} \times \mathbb{R}^{n-1}\right) \hat{\otimes} \mathcal{H}_{\xi_{n}}^{+} \hat{\otimes} \mathcal{H}_{d-1, \eta_{n}}^{-} .
\end{aligned}
$$

The spaces $\mathcal{K}_{1,0}^{m}, \mathcal{T}_{1,0}^{m}$ and $\mathcal{G}_{1,0}^{m}$ are denoted by Grubb in [19] as $S_{1,0}^{m-1}\left(\mathbb{R}^{n-1} \times\right.$ $\left.\mathbb{R}^{n-1} ; \mathcal{H}^{+}\right), S_{1,0}^{m}\left(\mathbb{R}^{n-1} \times \mathbb{R}^{n-1} ; \mathcal{H}_{d-1}^{-}\right)$, and $S_{1,0}^{m-1}\left(\mathbb{R}^{n-1} \times \mathbb{R}^{n-1} ; \mathcal{H}^{+} \hat{\otimes} \mathcal{H}_{d-1}^{-}\right)$. Rempel and Schulze denote them in [32] by $\mathfrak{K}^{m-1}\left(\mathbb{R}^{n-1} \times \mathbb{R}^{n}\right), \mathfrak{T}^{m, d}\left(\mathbb{R}^{n-1} \times \mathbb{R}^{n}\right)$ and $\mathfrak{B}^{m-1, d}\left(\mathbb{R}^{n-1} \times \mathbb{R}^{n+1}\right)$. They are Fréchet spaces with the topologies induced by the scaled functions. For fixed $\left(x^{\prime}, \xi^{\prime}\right)$ the symbols above define Wiener-Hopf operators. Hence we obtain an action in the normal direction:

$$
\begin{aligned}
& {\left[\mathrm{op}_{n} k\right]\left(x^{\prime}, \xi^{\prime}\right):=r^{+} \mathcal{F}_{\xi_{n} \rightarrow x_{n}}^{-1} k\left(x^{\prime}, \xi^{\prime} ; \xi_{n}\right): \mathbb{C} \rightarrow \mathcal{S}\left(\mathbb{R}_{+}\right),} \\
& {\left[\mathrm{op}_{n} t\right]\left(x^{\prime}, \xi^{\prime}\right):=I_{\xi_{n}}^{+} t\left(x^{\prime}, \xi^{\prime} ; \xi_{n}\right) \mathcal{F}_{y_{n} \rightarrow \xi_{n}} e^{+}: \mathcal{S}\left(\mathbb{R}_{+}\right) \rightarrow \mathbb{C} \text { and }} \\
& {\left[\mathrm{op}_{n} g\right]\left(x^{\prime}, \xi^{\prime}\right):=r^{+} \mathcal{F}_{\xi_{n} \rightarrow x_{n}}^{-1} I_{\eta_{n}}^{+} g\left(x^{\prime}, \xi^{\prime} ; \xi_{n}, \eta_{n}\right) \mathcal{F}_{y_{n} \rightarrow \eta_{n}} e^{+}: \mathcal{S}\left(\mathbb{R}_{+}\right) \rightarrow \mathcal{S}\left(\mathbb{R}_{+}\right),}
\end{aligned}
$$

where $I^{+}$is the plus-integral, see [19, p.166]. We can interpret op $k$, op $n$ and op $n$ as operator-valued symbols. Depending on the class there are several extensions possible.

Theorem 2.5 (Description by operator-valued symbols). Let $\mathbf{s} \in \mathbb{R}^{2}$ with $s_{1}>d-1 / 2$. The following maps are bounded and linear. 
(1) op $_{n}: \mathcal{G}_{1, \delta}^{m, 0}\left(\mathbb{R}^{n-1} \times \mathbb{R}^{n-1}\right) \rightarrow S_{1, \delta}^{m}\left(\mathbb{R}^{n-1} \times \mathbb{R}^{n-1} ; \mathcal{S}^{\prime}\left(\mathbb{R}_{+}\right), \mathcal{S}\left(\mathbb{R}_{+}\right)\right)$

(2) op $_{n}: \mathcal{G}_{1, \delta}^{m, d}\left(\mathbb{R}^{n-1} \times \mathbb{R}^{n-1}\right) \rightarrow S_{1, \delta}^{m}\left(\mathbb{R}^{n-1} \times \mathbb{R}^{n-1} ; H_{2}^{\mathbf{s}}\left(\mathbb{R}_{+}\right), \mathcal{S}\left(\mathbb{R}_{+}\right)\right)$

(3) op $_{n}: \mathcal{K}_{1, \delta}^{m}\left(\mathbb{R}^{n-1} \times \mathbb{R}^{n-1}\right) \rightarrow S_{1, \delta}^{m-1 / 2}\left(\mathbb{R}^{n-1} \times \mathbb{R}^{n-1} ; \mathbb{C}, \mathcal{S}\left(\mathbb{R}_{+}\right)\right)$

(4) op $_{n}: \mathcal{T}_{1, \delta}^{m, 0}\left(\mathbb{R}^{n-1} \times \mathbb{R}^{n-1}\right) \rightarrow S_{1, \delta}^{m+1 / 2}\left(\mathbb{R}^{n-1} \times \mathbb{R}^{n-1} ; \mathcal{S}^{\prime}\left(\mathbb{R}_{+}\right), \mathbb{C}\right)$

(5) op op $_{n} \mathcal{T}_{1, \delta}^{m, d}\left(\mathbb{R}^{n-1} \times \mathbb{R}^{n-1}\right) \rightarrow S_{1, \delta}^{m+1 / 2}\left(\mathbb{R}^{n-1} \times \mathbb{R}^{n-1} ; H_{2}^{\mathbf{s}}\left(\mathbb{R}_{+}\right), \mathbb{C}\right)$

We omit the proof, which is straightforward. We also need the description via symbolkernels. To this end we define

$$
\widetilde{\mathcal{K}}_{1, \delta}^{m}:=\mathcal{F}_{\xi_{n} \rightarrow x_{n}}^{-1} \mathcal{K}_{1, \delta}^{m}, \quad \widetilde{\mathcal{T}}_{1, \delta}^{m}:=\overline{\mathcal{F}}_{\xi_{n} \rightarrow y_{n}}^{-1} \mathcal{T}_{1, \delta}^{m, 0} \text { and } \widetilde{\mathcal{G}}_{1, \delta}^{m}:=\mathcal{F}_{\xi_{n} \rightarrow x_{n}}^{-1} \overline{\mathcal{F}}_{\eta_{n} \rightarrow y_{n}}^{-1} \mathcal{G}_{1, \delta}^{m, 0} .
$$

Theorem 2.6 (Description by symbol-kernels). The following assertions hold:

(i) For every operator-valued symbol $k \in S_{1, \delta}^{m}\left(\mathbb{R}^{n-1} \times \mathbb{R}^{n-1} ; \mathbb{C}, \mathcal{S}\left(\mathbb{R}_{+}\right)\right)$there exists a unique $\tilde{k} \in \tilde{\mathcal{K}}_{1, \delta}^{m}\left(\mathbb{R}^{n-1} \times \mathbb{R}^{n-1}\right)$, such that

$$
\left[k\left(x^{\prime}, \xi^{\prime}\right) c\right]\left(x_{n}\right)=\tilde{k}\left(x^{\prime}, \xi^{\prime} ; x_{n}\right) c, c \in \mathbb{C} .
$$

(ii) For every operator-valued symbol $t \in S_{1, \delta}^{m}\left(\mathbb{R}^{n-1} \times \mathbb{R}^{n-1} ; \mathcal{S}^{\prime}\left(\mathbb{R}_{+}\right)\right.$, $\left.\mathbb{C}\right)$ there exists a unique $\tilde{t} \in \widetilde{\mathcal{T}}_{1, \delta}^{m, 0}\left(\mathbb{R}^{n-1} \times \mathbb{R}^{n-1}\right)$, such that

$$
t\left(x^{\prime}, \xi^{\prime}\right) u=\int_{\mathbb{R}_{+}} \tilde{t}\left(x^{\prime}, \xi^{\prime} ; y_{n}\right) u\left(y_{n}\right) d y_{n}, u \in \mathcal{S}\left(\mathbb{R}_{+}\right) .
$$

(iii) For every operator-valued symbol $g \in S_{1, \delta}^{m}\left(\mathbb{R}^{n-1} \times \mathbb{R}^{n-1} ; \mathcal{S}^{\prime}\left(\mathbb{R}_{+}\right), \mathcal{S}\left(\mathbb{R}_{+}\right)\right)$there exists a unique $\tilde{g} \in \widetilde{\mathcal{G}}_{1, \delta}^{m}\left(\mathbb{R}^{n-1} \times \mathbb{R}^{n-1}\right)$, such that

$$
\left[g\left(x^{\prime}, \xi^{\prime}\right) u\right]\left(x_{n}\right)=\int_{\mathbb{R}_{+}} \tilde{g}\left(x^{\prime}, \xi^{\prime} ; x_{n}, y_{n}\right) u\left(y_{n}\right) d y_{n}, u \in \mathcal{S}\left(\mathbb{R}_{+}\right) .
$$

Proof See Theorems 3.7 and 3.9 in [35].

Corollary 2.7 The maps (1), (3), and (4) in Theorem 2.5 are bijections. The maps (2) and (5) are bijections onto their image, which is the set of all operators of the form

$$
\begin{aligned}
& \mathrm{op}_{n} g_{0}+\sum_{j=0}^{d-1} \mathrm{op}_{n} k_{j} \gamma_{j}^{+}, g_{0} \in \mathcal{G}_{1, \delta}^{m, 0}\left(\mathbb{R}^{n-1} \times \mathbb{R}^{n-1}\right), k_{j} \in \mathcal{K}_{1, \delta}^{m-j}\left(\mathbb{R}^{n-1} \times \mathbb{R}^{n-1}\right) \text { resp. } \\
& \mathrm{op}_{n} t_{0}+\sum_{j=0}^{d-1} s_{j} \gamma_{j}^{+}, t_{0} \in \mathcal{T}_{1, \delta}^{m, 0}\left(\mathbb{R}^{n-1} \times \mathbb{R}^{n-1}\right), s_{j} \in S_{1, \delta}^{m-j}\left(\mathbb{R}^{n-1} \times \mathbb{R}^{n-1}\right) .
\end{aligned}
$$

Proof We get from symbols to operator-valued symbols, to symbol-kernels, and back to symbols by Theorem 2.5, Theorem 2.6 and the Fourier transform. For non-zero class we use the fact $I^{+} \xi^{j} \mathcal{F} e^{+} \phi=(-i)^{j} \gamma_{j}^{+} \phi$. 


\subsection{Boundary symbols and operators}

We next define the space of boundary symbols of order $m$, class $d$ and Hörmander type $(1, \delta)$ by

$$
\mathcal{B} \mathcal{M}_{1, \delta}^{m, d}:=\left(\begin{array}{cc}
\mathcal{P}_{1, \delta}^{m}+\mathcal{G}_{1, \delta}^{m, d} & \mathcal{K}_{1, \delta}^{m} \\
\mathcal{T}_{1, \delta}^{m, d} & S_{1, \delta}^{m}
\end{array}\right)
$$

It is clear from Theorems 2.5 and 2.3 that the action of $b \in \mathcal{B M}_{1, \delta}^{m, d}$ in the normal direction defines a matrix of operator-valued symbols

$$
\mathrm{op}_{n}(b):=\left(\begin{array}{cc}
\mathrm{op}_{n}(p)_{+}+\mathrm{op}_{n}(g) & \mathrm{op}_{n}(k) \\
\mathrm{op}_{n}(t) & s
\end{array}\right)
$$

We write $B:=\mathrm{op}\left[\mathrm{op}_{n} b\right]$ for the associated operator. We denote the components of $B$ associated with $p, g, k, t$ and $s$ by $P_{+}, G, K, T$, and $S$, respectively. It is well-known that these operators form an algebra for Hörmander type $(1,0)$. The proof given in [35] extends to the case $(1, \delta)$ with obvious modifications.

Theorem 2.8 (Composition). Composition yields a bilinear and continuous map

$$
\mathcal{B} \mathcal{M}_{1, \delta}^{m, d} \times \mathcal{B} \mathcal{M}_{1, \delta}^{m^{\prime}, d^{\prime}} \rightarrow \mathcal{B} \mathcal{M}_{1, \delta}^{m+m^{\prime}, \max \left(m^{\prime}+d, d^{\prime}\right)}, \quad\left(b, b^{\prime}\right) \mapsto b \# b^{\prime},
$$

where \# is the Leibniz product of operator-valued symbols, given by the property that $\mathrm{op}\left(\mathrm{op}_{n} b\right)$ op $\left(\mathrm{op}_{n} b^{\prime}\right)=\mathrm{op}\left(\mathrm{op}_{n} b \# b^{\prime}\right)$. Moreover

$$
b \# b^{\prime} \equiv p p^{\prime}-p_{0} p_{0}^{\prime}+b_{0} \circ_{n} b_{0}^{\prime} \quad \bmod \mathcal{B M}_{1, \delta}^{m+m^{\prime}-(1-\delta), \max \left(m+d^{\prime}, d\right)} .
$$

Here the subscript 0 denotes the restriction to $x_{n}=0$ and $\circ_{n}$ denotes the point-wise composition, [19, Theorem 2.6.1].

The well-known mapping properties of Boutet de Monvel operators extend to operators of Hörmander type $(1, \delta)$. We refer to [18] for the proof of the following statement (in the case $\delta=0$ ).

Theorem 2.9 Let $b \in \mathcal{B M}_{1, \delta}^{m, d}$ and $s>d+1 / p-1$. Then

$$
B=\operatorname{op}\left(\operatorname{op}_{n} b\right): H_{p}^{s}\left(\mathbb{R}_{+}^{n}\right) \oplus B_{p}^{s-1 / p}\left(\mathbb{R}^{n-1}\right) \rightarrow H_{p}^{s-m}\left(\mathbb{R}_{+}^{n}\right) \oplus B_{p}^{s-m-1 / p}\left(\mathbb{R}^{n-1}\right)
$$

is bounded. The map $b \mapsto B$ is continuous.

Remark 2.10 The above calculus and the continuity properties naturally extend to the case of operators acting on vector bundles over compact manifolds with boundary. 


\section{The resolvent}

For the proof of Theorem 1.3, a suitable description of the resolvent $\left(A_{T}-\lambda\right)^{-1}$ is mandatory. We explain the key idea of how this description is derived in the simple example, where $A=-\Delta, T=\gamma_{0}$, and $v=1$. Here, the benefit is that we can point out the main ideas. Moreover, the majority of abstract arguments can be replaced by explicit computations.

In the article [2], Shmuel Agmon proved a priori estimates for solutions of the following boundary value problem with spectral parameter:

$$
\left\{\begin{array}{ll}
(1-\Delta-\lambda)_{+} u & =f \text { on } \mathbb{R}_{+}^{n} \\
\gamma_{0} u & =\phi \text { on } \mathbb{R}^{n-1}
\end{array} .\right.
$$

Writing $\lambda=\mu^{2} e^{i \theta}$, we observe that, given a solution $u$ of (3.1), the function $\tilde{u}:=$ $u \otimes e_{\mu}$ with $e_{\mu}(z)=e^{i \mu z}$ solves the elliptic boundary problem

$$
\left\{\begin{array}{lll}
\left(1-\Delta+e^{i(\pi+\theta)} D_{z}^{2}\right)_{+} \tilde{u} & =\tilde{f} \text { on } \mathbb{R}_{+}^{n+1} \\
\gamma_{0} \tilde{u} & =\tilde{\phi} \text { on } \mathbb{R}^{n}
\end{array}\right.
$$

with $\tilde{f}=f \otimes e_{\mu}$ and $\tilde{\phi}=\phi \otimes e_{\mu}$. For (3.2), a priori estimates are well-known, but for our purpose, they are not sufficient. However, the basic idea can be extended to provide a relation between the inverses of (3.2) and (3.1). The following three operators are of interest:

$$
\begin{aligned}
& Q_{\theta}:=r^{+} \mathcal{F}^{-1}\left(\langle\xi\rangle^{2}+e^{i(\pi+\theta)} \zeta^{2}\right)^{-1} \mathcal{F} e^{+}, \\
& K_{\theta}:=r^{+} \mathcal{F}^{\prime}-1 e^{-\kappa_{\theta}\left(\xi^{\prime}, \zeta\right) x_{n}} \mathcal{F}^{\prime} \text {, and } \\
& G_{\theta}:=-K_{\theta} \gamma_{0} Q_{\theta} \text {. }
\end{aligned}
$$

Here, $i \kappa_{\theta}\left(\xi^{\prime}, \zeta\right)$ is the root of the polynomial $\xi_{n} \mapsto a_{\theta}(\xi, \zeta):=\langle\xi\rangle^{2}+e^{i(\pi+\theta)} \zeta^{2}$, with positive imaginary part. Furthermore, $\mathcal{F}$ and $\mathcal{F}^{\prime}$, respectively, denote the Fourier transform with respect to all variables and the tangential variables, respectively. The identities $A_{\theta} Q_{\theta}=1, A_{\theta} K_{\theta}=0, \gamma_{0} K_{\theta}=1$, and $\gamma_{0}\left(Q_{\theta}+G_{\theta}\right)=0$ can be verified in a quick calculation. Therefore:

$$
\left(\begin{array}{c}
A_{\theta,+} \\
\gamma_{0}
\end{array}\right)^{-1}=\left(Q_{\theta,+}+G_{\theta} K_{\theta}\right) .
$$

The operators belong to Boutet de Monvel's calculus. We denote the symbols by lower case letters. The solution operators to Problem (3.2) and (3.1) are related. In order to reveal this relation, we need the following result.

Lemma 3.1 Let $p \in S_{1, \delta}^{m}\left(\mathbb{R}^{n} \times \mathbb{R}^{n+1} ; E, F\right)$. Then $p_{\mu}:=\left.p\right|_{\zeta=\mu} \in S_{1, \delta}^{m}\left(\mathbb{R}^{n} \times\right.$ $\left.\mathbb{R}^{n} ; E, F\right)$ and the associated operators are related as follows:

$$
P\left(u \otimes e_{\mu}\right)=\left(P_{\mu} u\right) \otimes e_{\mu}
$$


Proof For fixed $\mu, p_{\mu}$ is a symbol in view of the estimate:

$$
c\langle\xi\rangle \leq\langle\xi, \mu\rangle \leq C\langle\xi\rangle \text {, with } C=C(\mu) .
$$

The following formal computation can be justified using oscillatory integrals.

$$
\begin{aligned}
{\left[P\left(u \otimes e_{\mu}\right)\right](x, z) } & =\int e^{i x \xi+i z \zeta} p(x, \xi, \zeta)[\mathcal{F} u](\xi) \delta(\zeta-\mu) d \zeta d \xi \\
& =e^{i z \mu} \int e^{i x \xi} p(x, \xi, \mu)[\mathcal{F} u](\xi) d \xi \\
& =\left[\left(P_{\mu} u\right) \otimes e_{\mu}\right](x, z) .
\end{aligned}
$$

The above computation holds for each point, thus Eq. (3.4) holds.

Now, we verify that the function $u:=\left(Q_{\theta, \mu,+}+G_{\theta, \mu}\right) f+K_{\theta, \mu} \phi$ solves Problem (3.1) for given $f$ and $\phi$ :

$$
\begin{aligned}
{\left[(A-\lambda)_{+} u\right] \otimes e_{\mu} } & =A_{\theta,+}\left[u \otimes e_{\mu}\right]=A_{\theta,+}\left[\left(\left(Q_{\theta, \mu,+}+G_{\theta, \mu}\right) f+K_{\theta, \mu} \phi\right) \otimes e_{\mu}\right] \\
& \left.=A_{\theta}\left(Q_{\theta,+}+G_{\theta}\right)\left(f \otimes e_{\mu}\right)+A_{\theta} K_{\theta}\left(\phi \otimes e_{\mu}\right)\right] \stackrel{(3.3)}{=} f \otimes e_{\mu} . \\
{\left[\gamma_{0} u\right] \otimes e_{\mu} } & \left.=\gamma_{0}\left(Q_{\theta}+G_{\theta}\right)\left(f \otimes e_{\mu}\right)+\gamma_{0} K_{\theta}\left(\phi \otimes e_{\mu}\right)\right] \stackrel{(3.3)}{=} \phi \otimes e_{\mu} .
\end{aligned}
$$

Therefore, the inverse of the parameter-dependent problem can be constructed for the inverse of the associated extended problem. For $\lambda=\mu^{2} e^{i \theta}$ :

$$
\left(\begin{array}{c}
(A-\lambda)_{+} \\
\gamma_{0}
\end{array}\right)^{-1}=\left(Q_{\theta, \mu,+}+G_{\theta, \mu} K_{\theta, \mu}\right)
$$

What we are especially interested in is the left entry on the right hand side. Here, we observe:

$$
\left(Q_{\theta, \mu,+}+G_{\theta, \mu}\right) L_{p}\left(\mathbb{R}_{+}^{n}\right) \subset \mathcal{D}\left(A_{\gamma_{0}}\right):=\left\{u \in L_{p}\left(\mathbb{R}_{+}^{n}\right): A_{+} u \in L_{p}\left(\mathbb{R}_{+}^{n}\right), \gamma_{0} u=0\right\} .
$$

Therefore, we obtain an explicit formula for the resolvent:

$$
\left(A_{\gamma_{0}}-\lambda\right)^{-1}=Q_{\theta, \mu,+}+G_{\theta, \mu} \text {, on the ray } \lambda=e^{i \theta} \mu^{2} .
$$

The example encourages us to initially solve the extended problem:

$$
\begin{aligned}
\left(A+e^{i(\pi+\theta)} D_{z}^{2}\right)_{+} \tilde{u} & =\tilde{f} \\
T \tilde{u} & =\tilde{\phi} .
\end{aligned}
$$

In general, no explicit formulas for the inverse of the above problem exist. We will therefore replace the inverse by a parametrix and analyze the resulting error term. 
According to Eq. (3.4), the restriction $\zeta=\mu$ in Lemma 3.1 commutes with composition. Therefore, for an elliptic symbol $p$ with parametrix $p^{-\#}$ and remainder $r$ we obtain:

$$
P_{\mu} P_{\mu}^{-\#}=1+R_{\mu}
$$

To estimate the error term, we need to analyze the dependence on the parameters $\theta, \mu$ and thus on $\lambda$ of the operators above. The dependence on $\theta$ for $0<\vartheta \leq|\theta| \leq \pi$ is not essential. In fact, we obtain uniform estimates on operator norms that only depend on $\vartheta$. However, the dependence on $\mu$ is essential and will be discussed next.

\subsection{The dependence on the spectral parameter $\mu$}

We consider general Boutet de Monvel symbols which have a covariable $\zeta$ with no space dependence, i.e. they are constant with respect to the variable $z$. By restriction $\zeta=\mu$, we obtain again Boutet de Monvel symbols. The norms of the associated operators depend on the parameter $\mu$.

Theorem 3.2 Let $0 \leq \delta<1$.

(a) Let $p \in S_{1, \delta}^{-m}\left(\mathbb{R}^{n} \times \mathbb{R}^{n+1}\right)$ and $m \geq 0$. Then

$$
\left\|P_{\mu}\right\|_{\mathcal{L}\left(L_{p}\left(\mathbb{R}^{n}\right)\right)} \leq C|p|_{*}\langle\mu\rangle^{-m}
$$

(b) Let $g \in \mathcal{G}_{1, \delta}^{-m, 0}\left(\mathbb{R}^{n-1} \times \mathbb{R}^{n}\right)$ and $m>0$. Then

$$
\left\|G_{\mu}\right\|_{\mathcal{L}\left(L_{p}\left(\mathbb{R}_{+}^{n}\right)\right)} \leq C|g|_{*}\langle\mu\rangle^{-m}
$$

(c) Let $k \in \mathcal{K}_{1, \delta}^{-m}\left(\mathbb{R}^{n-1} \times \mathbb{R}^{n}\right)$ and $m \geq 0$. Then

$$
\left\|K_{\mu}\right\|_{\mathcal{L}\left(B_{p}^{-1 / p}\left(\mathbb{R}^{n-1}\right) ; L_{p}\left(\mathbb{R}_{+}^{n}\right)\right)} \leq C|k|_{*}\langle\mu\rangle^{-m}
$$

(d) Let $t \in \mathcal{T}_{1, \delta}^{-m, 0}\left(\mathbb{R}^{n-1} \times \mathbb{R}^{n}\right)$ and $m \geq 1$. Then

$$
\left\|T_{\mu}\right\|_{\mathcal{L}\left(L_{p}\left(\mathbb{R}_{+}^{n}\right) ; B_{p}^{1-1 / p}\left(\mathbb{R}^{n-1}\right)\right)} \leq C|t|_{*}\langle\mu\rangle^{-m+1} .
$$

Here, $C$ denotes a suitable constant and $|p|_{*},|g|_{*},|k|_{*},|t|_{*}$ suitable seminorms for $p, g, k$ and $t$, respectively.

Before we turn our attention to the proof, let us draw a conclusion from the above theorem which demonstrates its value.

Corollary 3.3 Let $m \geq m^{\prime} \geq 0$. Let $b \in \mathcal{B M}_{1, \delta}^{m, d}\left(\mathbb{R}^{n} \times \mathbb{R}^{n+1}\right)$ have a parametrix $b^{-\#} \in \mathcal{B M}_{1, \delta}^{-m^{\prime}, 0}\left(\mathbb{R}^{n} \times \mathbb{R}^{n+1}\right)$. Then $B_{\mu}$ is invertible for large $\mu$, and $\| B_{\mu}^{-1}-$ $B_{\mu}^{-\#} \|_{\mathcal{L}\left(L_{p}\left(\mathbb{R}_{+}^{n}\right) \oplus B_{P}^{-1 / p}\left(\mathbb{R}^{n-1}\right)\right)} \leq C|b|_{*}\langle\mu\rangle^{-N}$ for all $N \in \mathbb{N}_{0}$. 
Proof By assumption $b \# b^{-\#}=1-r$ with $r \in \mathcal{B} \mathcal{M}_{1, \delta}^{-\infty}\left(\mathbb{R}^{n} \times \mathbb{R}^{n+1}\right)$. As $B_{\mu} B_{\mu}^{-\#}=$ $1-R_{\mu}$, Theorem 3.2 implies that $\left\|R_{\mu}\right\| \leq C\langle\mu\rangle^{-N}$ for all $N \in \mathbb{N}_{0}$. For large $\mu$, the inverse of $1-R_{\mu}$ is given by a Neumann series. Therefore, $B_{\mu}$ has a right inverse for large $\mu$ :

$$
B_{\mu}^{-1}=B_{\mu}^{-\#}+B_{\mu}^{-\#} \sum_{j \in \mathbb{N}} R_{\mu}^{j}
$$

Clearly the second summand is rapidly decreasing in $\mu$. Similarly we obtain a left inverse.

For the proof of Theorem 3.2 we need the following observation. Since there is no dependence on the space variable $z$ we can interpret a pseudodifferential operator $P$ with symbol in $S_{1, \delta}^{0}\left(\mathbb{R}^{n} \times \mathbb{R}^{n+1}\right)$ as a pseudodifferential operator on the cylinder $\mathbb{R}^{n} \times \mathbb{S}_{L}$, where $\mathbb{S}_{L}$ is the circle with radius $L / 2 \pi$. Then we obtain:

Lemma 3.4 If $p \in S_{1, \delta}^{0}\left(\mathbb{R}^{n} \times \mathbb{R}^{n+1}\right)$, then for all $L>0$ we have

$$
P:=\operatorname{op}(p) \in \mathcal{L}\left(L_{p}\left(\mathbb{R}^{n} \times \mathbb{S}_{L}\right)\right) \text { and }\|P\|_{\mathcal{L}\left(L_{p}\left(\mathbb{R}^{n} \times \mathbb{S}_{L}\right)\right)} \leq C|p|_{*}
$$

Here $C$ is a constant independent of $L$.

Proof We first note that $P$ preserves $L$-periodicity:

$$
\begin{aligned}
{[P u](x, z+k L): } & =\int e^{i(x-y) \xi+i((z+k L)-w) \zeta} p(x, \xi, \zeta) u(y, w) d y d w d \xi d \zeta \\
& =\int e^{i(x-y) \xi+i(z-(w-k L)) \zeta} p(x, \xi, \zeta) u(y, w) d y d w d \xi d \zeta \\
& =\int e^{i(x-y) \xi+i(z-\tilde{w}) \zeta} p(x, \xi, \zeta) u(y, \tilde{w}) d y d \tilde{w} d \xi d \zeta \\
& =[P u](x, z), \quad u \in C_{c}^{\infty}\left(\mathbb{R}^{n} \times \mathbb{S}_{L}\right) .
\end{aligned}
$$

We identify $u \in L_{p}\left(\mathbb{R}^{n} \times \mathbb{S}_{L}\right)$ with an $L$-periodic function by letting

$$
u=\sum_{j \in \mathbb{Z}} u_{j} \text { with } u_{j}(x, z):=\left.u\right|_{\mathbb{R}^{n} \times[-L / 2, L / 2]}(x, z-L j) .
$$

Note that for every $j \in \mathbb{Z}$ we have $u_{j} \in L_{p}\left(\mathbb{R}^{n} \times \mathbb{R}\right)$ and $\left\|u_{j}\right\|_{L_{p}\left(\mathbb{R}^{n} \times \mathbb{R}\right)}=$ $\|u\|_{L_{p}\left(\mathbb{R}^{n} \times \mathbb{S}_{L}\right)}$. The integral kernel $k=k(x, z, y, w)$ of the pseudodifferential operator $P$ is given by

$$
k(x, z, y, w)=\iint e^{i(x-y) \xi+i(z-w) \zeta} p(x, \xi, \zeta) d \xi d \zeta .
$$

Since $p$ is of order zero, we obtain the estimate

$$
|k(x, z, y, w)| \leq C|p|_{*}\left(|x-y|^{2}+|z-w|^{2}\right)^{-l / 2}
$$


for all even $l \in \mathbb{N}$ with $l>n$ with a suitable seminorm $|p|_{*}$ for $p$. For $|j| \geq 2$, $z \in[-L / 2, L / 2]$ and $w \in \operatorname{supp} u_{j}$ we have $|z-w| \geq(j-1) L$, hence

$$
\begin{gathered}
|k(x, z, y, w)| \leq C|p|_{*}\left(|x-y|^{2}+(|j|-1)^{2} L^{2}\right)^{-(n+2) / 2} \\
\leq C|p|_{*}((|j|-1) L)^{-(n+2)}\langle|x-y| /(|j|-1) L\rangle^{-(n+2)} .
\end{gathered}
$$

We write $\chi_{j}$ for the indicator function of $[-L / 2+j L, L / 2+j L]$. A quick computation shows that

$$
\begin{aligned}
& \int \chi_{0}(z)|k(x, z, y, w)| \chi_{j}(w) d w d y \leq C|p|_{*} L^{-1}(|j|-1)^{-2} \text { and } \\
& \int \chi_{0}(z)|k(x, z, y, w)| \chi_{j}(w) d z d x \leq C|p|_{*} L^{-1}(|j|-1)^{-2} \text {. }
\end{aligned}
$$

Hence we get $L_{p}$-estimates by Schur's test, for Schur's test we refer to [39, Theorem 0.3.1]. More explicitly:

$$
\begin{aligned}
& \left\|P u_{j}\right\|_{L_{p}\left(\mathbb{R}^{n} \times \mathbb{S}_{L}\right)}=\left\|\chi_{0} P \chi_{j} u_{j}\right\|_{L_{p}\left(\mathbb{R}^{n} \times \mathbb{R}\right)} \\
& \quad \leq C|p|_{*} L^{-1}(|j|-1)^{-2}\left\|u_{j}\right\|_{L_{p}\left(\mathbb{R}^{n} \times \mathbb{R}\right)}=C|p|_{*} L^{-1}(|j|-1)^{-2}\|u\|_{L_{p}\left(\mathbb{R}^{n} \times \mathcal{S}_{L}\right)}
\end{aligned}
$$

In particular the right hand side is summable, and for $L \geq 1$ we obtain

$$
\begin{aligned}
& \|P u\|_{L_{p}\left(\mathbb{R}^{n} \times \mathbb{S}_{L}\right)} \\
& =\sum_{j \in\{-1,0,1\}}\left\|P u_{j}\right\|_{L_{p}\left(\mathbb{R}^{n} \times \mathbb{S}_{L}\right)}+\sum_{|j| \geq 2}\left\|P u_{j}\right\|_{L_{p}\left(\mathbb{R}^{n} \times \mathbb{S}_{L}\right)} \\
& \leq C\left(3|p|_{*}\|u\|_{L_{p}\left(\mathbb{R}^{n} \times \mathbb{S}_{L}\right)}+2 \sum_{j \in \mathbb{N}} j^{-2}|p|_{*}\|u\|_{L_{p}\left(\mathbb{R}^{n} \times \mathbb{S}_{L}\right)}\right) \\
& \leq C|p|_{*}\|u\|_{L_{p}\left(\mathbb{R}^{n} \times \mathbb{S}_{L}\right)}
\end{aligned}
$$

We still need to prove that the bound also holds for $L<1$. Choose $N \in \mathbb{N}$ so large that $N L \geq 1$, and consider an $L$-periodic function as an $N L$-periodic function. We have $\|u\|_{L_{p}\left(\mathbb{R}^{n} \times \mathbb{S}_{N L}\right)}=N^{1 / p}\|u\|_{L_{p}\left(\mathbb{R}^{n} \times \mathbb{S}_{L}\right)}$ and hence, by the above argument,

$$
\begin{aligned}
& \|P u\|_{L_{p}\left(\mathbb{R}^{n} \times \mathbb{S}_{L}\right)}=N^{-1 / p}\|P u\|_{L_{p}\left(\mathbb{R}^{n} \times \mathbb{S}_{N L}\right)} \\
& \quad \leq C|p|_{*} N^{-1 / p}\|u\|_{L_{p}\left(\mathbb{R}^{n} \times \mathbb{S}_{N L}\right)}=C|p|_{*}\|u\|_{L_{p}\left(\mathbb{R}^{n} \times \mathbb{S}_{L}\right)}
\end{aligned}
$$

for a constant $C$ independent of $N L$.

Proof of Theorem 3.2 Let us first assume that $p \in S_{1, \delta}^{0}\left(\mathbb{R}^{n} \times \mathbb{R}^{n+1}\right)$. We write $e_{\mu}$ for the $2 \pi / \mu$-periodic function $\left[x \mapsto e^{i \mu x}\right]$. For $u \in L_{p}\left(\mathbb{R}^{n}\right)$ we take the $L_{p}$-norm of both sides of Eq. (3.4):

$$
\begin{aligned}
& \left\|P\left(u \otimes e_{\mu}\right)\right\|_{L_{p}\left(\mathbb{R}^{n} \times \mathbb{S}_{2 \pi / \mu}\right)} \\
& \quad=\left\|\left[P_{\mu} u\right] \otimes e_{\mu}\right\|_{L_{p}\left(\mathbb{R}^{n} \times \mathbb{S}_{2 \pi / \mu}\right)}=\left\|P_{\mu} u\right\|_{L_{p}\left(\mathbb{R}^{n}\right)}\left\|e_{\mu}\right\|_{L_{p}\left(\mathbb{S}_{2 \pi / \mu}\right)} .
\end{aligned}
$$


Since $P$ is of order zero, Lemma 3.4 yields

$$
\begin{aligned}
& \left\|P_{\mu} u\right\|_{L_{p}\left(\mathbb{R}^{n}\right)}\left\|e_{\mu}\right\|_{L_{p}\left(\mathbb{S}_{2 \pi / \mu}\right)} \\
& \quad \leq C|p|_{*}\left\|u \otimes e_{\mu}\right\|_{L_{p}\left(\mathbb{R}^{n} \times \mathbb{S}_{2 \pi / \mu}\right)}=C|p|_{*}\|u\|_{L_{p}\left(\mathbb{R}^{n}\right)}\left\|e_{\mu}\right\|_{L_{p}\left(\mathbb{S}_{2 \pi / \mu}\right)},
\end{aligned}
$$

and part (a) follows for $m=0$. For $m<0$ we can use what we did so far to reduce to the case $p(x, \xi, \mu)=\langle\xi, \mu\rangle^{-m}$. But for this symbol the statement is a consequence of the $L_{p}$-mapping property of pseudodifferential operators and the following simple estimates.

$$
\left|D_{\xi}^{\alpha}\langle\xi, \mu\rangle^{-m}\right| \leq C_{\alpha}\langle\xi, \mu\rangle^{-m-|\alpha|} \leq C_{\alpha}\langle\mu\rangle^{-m}\langle\xi\rangle^{-|\alpha|}
$$

Now for part (b). We recall that $\tilde{g} \in \widetilde{\mathcal{G}}_{1, \delta}^{m, 0}\left(\mathbb{R}^{n-1} \times \mathbb{R}^{n}\right)$ satisfies the estimates

$$
\begin{gathered}
\left\|\left[D_{x_{n}}^{l} x_{n}^{l^{\prime}} D_{y_{n}}^{l^{\prime \prime}} y_{n}^{l^{\prime \prime \prime}} D_{\xi^{\prime}}^{\alpha} D_{x^{\prime}}^{\beta} \tilde{g}_{\mu}\right]\left(x^{\prime}, \xi^{\prime}, x_{n}, \cdot\right)\right\|_{L_{1}\left(\mathbb{R}_{+}\right)} \leq C|g|_{*}\left\langle\xi^{\prime}, \mu\right\rangle^{m-|\alpha|+\delta|\beta|+l-l^{\prime}+l^{\prime \prime}-l^{\prime \prime \prime}} \\
\left\|\left[D_{x_{n}}^{l} x_{n}^{l^{\prime}} D_{y_{n}}^{l^{\prime \prime}} y_{n}^{l^{\prime \prime \prime}} D_{\xi^{\prime}}^{\alpha} D_{x^{\prime}}^{\beta} \tilde{g}_{\mu}\right]\left(x^{\prime}, \xi^{\prime}, \cdot, y_{n}\right)\right\|_{L_{1}\left(\mathbb{R}_{+}\right)} \leq C|g|_{*}\left\langle\xi^{\prime}, \mu\right\rangle^{m-|\alpha|+\delta|\beta|+l-l^{\prime}+l^{\prime \prime}-l^{\prime \prime \prime}} .
\end{gathered}
$$

So Schur's test implies that $\| D_{\xi^{\prime}}^{\alpha}$ op $_{n} \tilde{g}_{\mu} \|_{\mathcal{L}\left(L_{p}\left(\mathbb{R}_{+}\right)\right)} \leq C|g|_{*}\left\langle\xi^{\prime}, \mu\right\rangle^{m-|\alpha|}$. We are interested in the integral kernel

$$
\begin{aligned}
K\left(x^{\prime}, y^{\prime}, \mu\right) & =\int e^{i\left(x^{\prime}-y^{\prime}\right) \xi^{\prime}} \operatorname{op}_{n} \tilde{g}_{\mu}\left(x^{\prime}, \xi^{\prime}\right) d \xi^{\prime} \\
& =\int L^{N}\left(e^{i\left(x^{\prime}-y^{\prime}\right) \xi^{\prime}}-1\right) \text { op }_{n} \tilde{g}_{\mu}\left(x^{\prime}, \xi^{\prime}\right) d \xi^{\prime}
\end{aligned}
$$

with $N \in \mathbb{N}$ and $L:=\sum_{|\alpha|=1} \frac{\left(x^{\prime}-y^{\prime}\right)^{\alpha}}{\left|x^{\prime}-y^{\prime}\right|^{2}} D_{\xi^{\prime}}^{\alpha}$. We take $N=n-1$ and use the fact that $\left|e^{i t}-1\right| \leq 2|t|^{\theta}$ for $0<\theta<\min (1,|m|)$, to get

$$
\begin{aligned}
& \left\|K\left(x^{\prime}, y^{\prime}, \mu\right)\right\|_{\mathcal{L}\left(L_{p}\left(\mathbb{R}_{+}\right)\right)} \leq C|g|_{*}\left|x^{\prime}-y^{\prime}\right|^{-n+1+\theta} \\
& \int\left|\xi^{\prime}\right|^{\theta}\left\langle\xi^{\prime}, \mu\right\rangle^{-m-n+1} d \xi^{\prime} \leq C|g|_{*}\left|x^{\prime}-y^{\prime}\right|^{-n+1+\theta}\langle\mu\rangle^{-m+\theta}
\end{aligned}
$$

Choosing $N=n$ we obtain $\left\|K\left(x^{\prime}, y^{\prime}, \mu\right)\right\|_{\mathcal{L}\left(L_{p}\left(\mathbb{R}_{+}\right)\right)} \leq C|g|_{*}\left|x^{\prime}-y^{\prime}\right|^{-n}\langle\mu\rangle^{-m-1}$. The first estimate for $\langle\mu\rangle\left|x^{\prime}-y^{\prime}\right| \leq 1$ and the second for $\langle\mu\rangle\left|x^{\prime}-y^{\prime}\right|>1$ imply

$$
\begin{aligned}
& \left\|K\left(x^{\prime}, \cdot, \mu\right)\right\|_{L_{1}\left(\mathbb{R}^{n-1} ; \mathcal{L}\left(L_{p}\left(\mathbb{R}_{+}\right)\right)\right)} \leq C|g|_{*}\langle\mu\rangle^{-m} \text { and } \\
& \left\|K\left(\cdot, y^{\prime}, \mu\right)\right\|_{L_{1}\left(\mathbb{R}^{n-1} ; \mathcal{L}\left(L_{p}\left(\mathbb{R}_{+}\right)\right)\right)} \leq C|g|_{*}\langle\mu\rangle^{-m} .
\end{aligned}
$$


In fact, this follows from the the identities

$$
\begin{aligned}
& \int_{\langle\mu\rangle\left|x^{\prime}-y^{\prime}\right| \leq 1}\left|x^{\prime}-y^{\prime}\right|^{-n+1+\theta}\langle\mu\rangle^{\theta} d x^{\prime} \\
& \quad=\int_{\langle\mu\rangle\left|x^{\prime}-y^{\prime}\right| \leq 1}\left(\langle\mu\rangle\left|x^{\prime}-y^{\prime}\right|\right)^{-n+1+\theta}\langle\mu\rangle^{n-1} d x^{\prime}=\int_{|w| \leq 1}|w|^{-n+1+\theta} d w<\infty \\
& \text { and } \\
& \int_{\langle\mu\rangle\left|x^{\prime}-y^{\prime}\right| \geq 1}\left|x^{\prime}-y^{\prime}\right|^{-n}\langle\mu\rangle^{-1} d x^{\prime}=\int_{|w| \geq 1}|w|^{-n} d w<\infty .
\end{aligned}
$$

Hence the assertion follows with Schur's test.

For part (c): We recall the well-known fact that every potential operator $K$ can be written as $r^{+} P \tilde{\gamma}_{0}^{*}$, where $P$ is a pseudodifferential operator of order $-m-1$ whose symbol-kernel is given by $\tilde{p}=E \tilde{k} ; E$ is Seeley's extension operator applied to $x_{n}$, and $\tilde{\gamma}_{0}^{*}$ is the adjoint to the evaluation $\tilde{\gamma}_{0}: H_{p^{\prime}}^{s}\left(\mathbb{R}^{n}\right) \rightarrow B_{p^{\prime}}^{s-1 / p^{\prime}}\left(\mathbb{R}^{n-1}\right), s>1 / p^{\prime}$. It is clear that $K_{\mu}=r^{+} P_{\mu} \tilde{\gamma}_{0}^{*}$. The map

$$
S_{1,0}^{-1}\left(\mathbb{R}^{n} \times \mathbb{R}^{n+1}\right) \ni\langle\xi, \zeta\rangle^{-1} \mapsto\langle\xi, \mu\rangle^{-1} \in S_{1,0}^{-1}\left(\mathbb{R}^{n} \times \mathbb{R}^{n}\right)
$$

is uniformly bounded with respect to $\mu$. In view of the continuity of $\tilde{\gamma}_{0}^{*}$ from $B_{p}^{-1 / p}\left(\mathbb{R}^{n-1}\right)$ to $H_{p}^{-1}\left(\mathbb{R}^{n}\right)$ we have

$$
\left\|\operatorname{op}\left(\langle\xi, \mu\rangle^{-1}\right) \tilde{\gamma}_{0}^{*}\right\|_{\mathcal{L}\left(B_{p}^{-1 / p}\left(\mathbb{R}^{n-1}\right), L_{p}\left(\mathbb{R}^{n}\right)\right)} \leq C
$$

Define $q=p \#\langle\xi, \zeta\rangle^{1} \in S_{1, \delta}^{-m}\left(\mathbb{R}^{n} \times \mathbb{R}^{n+1}\right)$. By part (a)

$$
\left\|Q_{\mu}\right\|_{\mathcal{L}\left(L_{p}\left(\mathbb{R}^{n}\right)\right)} \leq C|q|_{*}\langle\mu\rangle^{-m} \leq C|k|_{*}\langle\mu\rangle^{-m}
$$

The estimate for $K_{\mu}$ follows.

For part (d) we use a similar approach. We write $T=\gamma_{0} P e^{+}$, where $P$ is a pseudodifferential operator of order $m$ with symbol-kernel $\tilde{p}=E \tilde{t}$. Clearly $T_{\mu}=\gamma_{0} P_{\mu} e^{+}$. By the same argument as in part (c) we have

$$
\left\|\gamma_{0} \operatorname{op}\left(\langle\xi, \mu\rangle^{-1}\right)\right\|_{\mathcal{L}\left(L_{p}\left(\mathbb{R}^{n}\right) ; B_{p}^{1-1 / p}\left(\mathbb{R}^{n-1}\right)\right)} \leq C
$$

Define $q=\langle\xi, \zeta\rangle^{1} \# p \in S_{1, \delta}^{-m+1}\left(\mathbb{R}^{n} \times \mathbb{R}^{n+1}\right)$. By part (a)

$$
\left\|Q_{\mu}\right\|_{\mathcal{L}\left(L_{p}\left(\mathbb{R}^{n}\right)\right)} \leq C|q|_{*}\langle\mu\rangle^{-m+1} \leq C|k|_{*}\langle\mu\rangle^{-m+1} .
$$

The estimate for $T_{\mu}$ follows. 


\subsection{The principal symbol of the degenerate singular Green operator}

We will now apply Agmon's trick to our problem. We introduce the operator $A_{\theta}:=$ $A+e^{i \theta} D_{z}^{2}$ acting on $\mathbb{R}_{+}^{n} \times \mathbb{R}$. The symbol of $A_{\theta}$ is $a_{\theta}(x, \xi, \zeta)=a(x, \xi)+e^{i \theta} \zeta^{2} \in$ $S_{1,0}^{2}\left(\mathbb{R}^{n} \times \mathbb{R}^{n+1}\right)$, where $a(x, \xi)$ is the symbol of $A$. Assuming that $a$ is homogeneous of degree 2, there exists a constant $c=c(M, \vartheta)$ such that for all $0<\vartheta \leq|\theta| \leq \pi$ the estimate $\left|a_{\theta}(x, \xi, \zeta)\right| \geq c|\xi, \zeta|^{2}$ holds. In particular, $A_{\theta}$ is elliptic. After possibly replacing $A$ by $A+c$ for some positive constant $c$ we may and will assume that the Dirichlet problem for $A_{\theta}$ is invertible. In the introduction we already pointed out that the solution operator to the Dirichlet problem is an operator in the Boutet de Monvel calculus, i.e.

$$
\left(\begin{array}{c}
\left(A_{\theta}\right)_{+} \\
\gamma_{0}
\end{array}\right)^{-1}=\left(Q_{\theta,+}+G_{\theta}^{D} K_{\theta}^{D}\right)
$$

We will need the principal symbols of the operators $G_{\theta}^{D}$ and $K_{\theta}^{D}$ and collect the results to fix some notation.

Remark 3.5 (a) For fixed $\left(x^{\prime}, \xi^{\prime}\right)$, the restriction to the boundary of the principal symbol of $A_{\theta}$ is a polynomial of degree two in $\xi_{n}$. It therefore has two roots, say $\pm i \kappa_{\theta}^{ \pm}\left(x^{\prime}, \xi^{\prime}, \zeta\right)$, with $\operatorname{Re} \kappa_{\theta}^{ \pm} \geq 0$.

(b) We have $\kappa_{\theta}^{ \pm} \in S_{1,0}^{1}\left(\mathbb{R}^{n-1} \times \mathbb{R}^{n}\right)$. Both are strongly elliptic, i.e. $\operatorname{Re} \kappa_{\theta}^{ \pm} \geq \omega\left|\xi^{\prime}, \zeta\right|$ for suitable $\omega>0$.

(c) The principal symbol of $K_{\theta}^{D} \in \mathcal{K}_{1,0}^{0}\left(\mathbb{R}^{n-1} \times \mathbb{R}^{n}\right)$ is $\left(\kappa_{\theta}^{+}+i \xi_{n}\right)^{-1}$.

(d) The principal symbol of $G_{\theta}^{D} \in \mathcal{G}_{1,0}^{-2,0}\left(\mathbb{R}^{n-1} \times \mathbb{R}^{n}\right)$ is $a_{n n}^{-1}\left(\kappa_{\theta}^{+}+\kappa_{\theta}^{-}\right)^{-1}\left(\kappa_{\theta}^{+}+i \xi_{n}\right)^{-1}\left(\kappa_{\theta}^{-}-i \eta_{n}\right)^{-1}$.

For details see [21, Section 2].

For large $-\lambda=e^{i \theta} \mu^{2}$ define

$$
G_{\theta}^{T}:=-K_{\theta}^{D}\left(T K_{\theta}^{D}\right)^{-\#} T\left(\left(A_{\theta}^{-1}\right)_{+}+G_{\theta}^{D}\right) .
$$

The operator $G_{\lambda}^{T}$ defined in (1.9) coincides with $G_{\theta, \mu}^{T} \bmod \mathcal{O}\left(\langle\lambda\rangle^{-N}\right.$ ) for all $N \in \mathbb{N}$, as operators in $L_{p}\left(\mathbb{R}_{+}^{n}\right)$. Moreover, let $G_{\theta}^{T \text {,* }}$ be any operator with the same principal symbol as $G_{\theta}^{T}$. Then according to Theorem 3.2 and the lemma below, $G_{\lambda}^{T}=G_{\theta, \mu}^{T, *}$ $\bmod o\left(\langle\lambda\rangle^{-1}\right)$, as operators on $L_{p}\left(\mathbb{R}_{+}^{n}\right)$.

Lemma 3.6 The operator $G_{\theta}^{T}$ is a singular Green operator with symbol $g_{\theta}^{T} \in$ $\mathcal{G}_{1,1 / 2}^{-2,0}\left(\mathbb{R}^{n-1} \times \mathbb{R}^{n}\right)$ and principal symbol

$g_{\theta(-2)}^{T}\left(x^{\prime}, \xi^{\prime}, \zeta ; \xi_{n}, \eta_{n}\right)=s_{\theta}^{T}\left(x^{\prime}, \xi^{\prime}, \zeta\right)\left(\kappa_{\theta}^{+}\left(x^{\prime}, \xi^{\prime}, \zeta\right)+i \xi_{n}\right)^{-1}\left(\kappa_{\theta}^{-}\left(x^{\prime}, \xi^{\prime}, \zeta\right)-i \eta_{n}\right)^{-1}$

for suitable $s_{\theta}^{T} \in S_{1,1 / 2}^{-1}\left(\mathbb{R}^{n-1} \times \mathbb{R}^{n}\right)$. The corresponding symbol-kernel is

$$
\tilde{g}_{\theta(-2)}^{T}\left(x^{\prime}, \xi^{\prime}, \zeta ; x_{n}, y_{n}\right)=s_{\theta}^{T}\left(x^{\prime}, \xi^{\prime}, \zeta\right) e^{-\kappa_{\theta}^{+}\left(x^{\prime}, \xi^{\prime}, \zeta\right) x_{n}} e^{-\kappa_{\theta}^{-}\left(x^{\prime}, \xi^{\prime}, \zeta\right) y_{n}} .
$$


Proof Modulo smoothing operators $G_{\theta}^{T}$ is the composition of the potential operator $K_{\theta}^{D}$, a parametrix $S_{\theta}^{-\#}$ to the pseudodifferential operator $S_{\theta}:=T K_{\theta}^{D}$ on the boundary, multiplication by the function $\varphi_{1}$ introduced in (1.2) and the trace operator $\gamma_{1}\left(Q_{\theta,+}+\right.$ $\left.G_{\theta}^{D}\right)$. Note that $Q_{\theta,+}+G_{\theta}^{D}$ maps into the kernel of $\gamma_{0}$ so that there is no contribution from $\varphi_{0} \gamma_{0}$. Hence the principal symbol of $G_{\theta}^{T}$ is given by multiplication of the principal symbols of these operators. For the proof of the lemma it is therefore sufficient to combine the following three statements.

(i) $K_{\theta}^{D}=$ op $k_{\theta}$ with $k_{\theta} \in \mathcal{K}_{1,0}^{0}\left(\mathbb{R}^{n-1} \times \mathbb{R}^{n}\right)$ and principal symbol

$$
k_{\theta(0)}\left(x^{\prime}, \xi^{\prime}, \zeta, \xi_{n}\right)=\left(\kappa_{\theta}^{+}\left(x^{\prime}, \xi^{\prime}, \zeta\right)+i \xi_{n}\right)^{-1},
$$

which is Remark 3.5(c).

(ii) The symbol $s_{\theta}^{-\#} \# \varphi_{1}$ of $S_{\theta}^{-\#} \varphi_{1}$ is an element of $S_{1,1 / 2}^{-1}\left(\mathbb{R}^{n-1} \times \mathbb{R}^{n}\right)$. This is the content of Lemma 3.8, below.

(iii) $\gamma_{1}\left(Q_{\theta,+}+G_{\theta}^{D}\right)=$ op $t_{\theta}$ with $t_{\theta} \in \mathcal{T}_{1,0}^{-1,0}$ and principal symbol

$$
t_{\theta(-1)}\left(x^{\prime}, \xi^{\prime}, \zeta, \xi_{n}\right)=-a_{n}\left(x^{\prime}\right)^{-1}\left(\kappa_{\theta}^{-}\left(x^{\prime}, \xi^{\prime}, \zeta\right)-i \xi_{n}\right)^{-1}
$$

which follows from Remark 3.5 and the composition rules.

\subsection{The parametrix on the boundary}

We recall a sufficient condition for the existence of a parametrix.

Theorem 3.7 (Parametrix). Let $m \geq 0$ and $p \in S_{1,0}^{m}\left(\mathbb{R}^{n} \times \mathbb{R}^{n}\right)$. Suppose there exists $a 0 \leq \delta<1$, such that for sufficiently large $|\xi|$ we have the estimates

$$
\begin{aligned}
& |p(x, \xi)| \geq c \text { and } \\
& \left|\partial_{x}^{\beta} \partial_{\xi}^{\alpha} p(x, \xi) p(x, \xi)^{-1}\right| \leq C\langle\xi\rangle^{-|\alpha|+\delta|\beta|} \text { for all } \alpha, \beta \in \mathbb{N}_{0}^{n} .
\end{aligned}
$$

Then there exists a parametrix $p^{-\#} \in S_{1, \delta}^{0}\left(\mathbb{R}^{n} \times \mathbb{R}^{n}\right)$, i.e.,

$$
p^{-\#} \# p=1+r_{1} \text { and } p \# p^{-\#}=1+r_{2} \text {, }
$$

with $r_{1}, r_{2} \in S^{-\infty}\left(\mathbb{R}^{n} \times \mathbb{R}^{n}\right)$.

Proof See [28, Chapter 2, Theorem 5.4].

Lemma 3.8 The operator $S_{\theta}:=T K_{\theta}^{D}$ has a parametrix with symbol $s_{\theta}^{-\#}$ in $S_{1,1 / 2}^{0}\left(\mathbb{R}^{n-1} \times \mathbb{R}^{n}\right)$. Moreover $s_{\theta}^{-\#} \# \varphi_{1} \in S_{1,1 / 2}^{-1}\left(\mathbb{R}^{n-1} \times \mathbb{R}^{n}\right)$.

Before going into the proof let us point out that the difference between the Robin and the degenerate boundary value problem is the order of the operator $S_{\theta}$ which here 
is zero due to the zeros of $\varphi_{1}$ and the resulting loss of ellipticity. The key observation is that we gain back the loss in order by composing with the multiplication operator $\varphi_{1}$.

Proof We want to show that the symbol of $S_{\theta}=T K_{\theta}^{D}$ satisfies inequalities (3.11) and (3.12). Write

$$
T K_{\theta}^{D}=\varphi_{1} \gamma_{1} K_{\theta}^{D}+\varphi_{0} \gamma_{0} K_{\theta}^{D}=\varphi_{1} \Pi_{\theta}+\varphi_{0},
$$

where $\Pi_{\theta}:=\gamma_{1} K_{\theta}^{D}$ is the Dirichlet-to-Neumann operator. It is well-known and a consequence of Remark 3.5(c) that its symbol $\pi_{\theta}$ is an element of $S_{1,0}^{1}\left(\mathbb{R}^{n-1} \times \mathbb{R}^{n}\right)$; its principal symbol is $\kappa_{\theta}^{+}$. By Remark 3.5(b) we have Re $\pi_{\theta} \geq 1$ for sufficiently large $|\xi, \zeta|$. Hence, the symbol $s_{\theta}$ of $S_{\theta}$ satisfies:

$$
\left|s_{\theta}\right| \geq\left|\operatorname{Re}\left(\varphi_{1} \pi_{\theta}+\varphi_{0}\right)\right|=\varphi_{1} \operatorname{Re} \pi_{\theta}+\varphi_{0} \geq \varphi_{1}+\varphi_{0} \geq c>0 .
$$

The constant $c$ exists by assumption. We have to verify the estimates

$$
\left|\partial_{x^{\prime}}^{\beta} \partial_{\xi^{\prime}}^{\alpha} \partial_{\zeta^{\prime}}^{l} s_{\theta} s_{\theta}^{-1}\right| \leq\left\langle\xi^{\prime}, \zeta\right\rangle^{-|\alpha|-l+|\beta| / 2} \text { for all } \alpha, \beta \in \mathbb{N}_{0}^{n-1}, l \in \mathbb{N}_{0}
$$

The estimate is trivial for $|\beta| \geq 2$, as $s_{\theta} \in S_{1,0}^{1}\left(\mathbb{R}^{n-1} \times \mathbb{R}^{n}\right)$ and $\left|s_{\theta}^{-1}\right| \leq c^{-1}$ by Eq. (3.13). Equation (3.13) also shows that $\left(\varphi_{1} \pi_{\theta}\right)^{k / 2} s_{\theta}^{-1}$ is bounded for $k=1,2$. The ellipticity of $\pi_{\theta}$ implies that $\left|\pi_{\theta}\right|^{-k / 2} \lesssim\left\langle\xi^{\prime}, \zeta\right\rangle^{-k / 2}$. We obtain the remaining estimates:

$$
\left|\partial_{\xi^{\prime}}^{\alpha} \partial_{\zeta^{\prime}}^{l} s_{\theta} s_{\theta}^{-1}\right| \equiv\left|\varphi_{1} \partial_{\xi^{\prime}}^{\alpha} \partial_{\zeta}^{l} \pi_{\theta} s_{\theta}^{-1}\right|=\left|\partial_{\xi^{\prime}}^{\alpha} \partial_{\zeta}^{l} \pi_{\theta} \pi_{\theta}^{-1}\right|\left|\varphi_{1} \pi_{\theta}\left(\varphi_{1} \pi_{\theta}+\varphi_{0}\right)^{-1}\right| \lesssim\left\langle\xi^{\prime}\right\rangle^{-|\alpha|-l}
$$

and with the help of the inequality $\left|\partial_{x_{j}} \varphi_{1}(x)\right|^{2} \leq\left\|\varphi_{1}^{\prime \prime}\right\|_{\infty}\left|\varphi_{1}(x)\right|$ :

$$
\begin{aligned}
\left|\partial_{x_{j}} \partial_{\xi^{\prime}}^{\alpha} \partial_{\zeta}^{l} s_{\theta} s_{\theta}^{-1}\right| & \equiv\left|\partial_{x_{j}} \varphi_{1} \partial_{\xi^{\prime}}^{\alpha} \partial_{\zeta}^{l} \pi_{\theta} s_{\theta}^{-1}\right| \lesssim\left\|\varphi_{1}^{\prime \prime}\right\|_{\infty}^{1 / 2}\left|\left(\varphi_{1} \pi_{\theta}\right)^{1 / 2} s_{\theta}^{-1}\right|\left|\pi_{\theta}\right|^{-1 / 2}\left|\partial_{\xi^{\prime}}^{\alpha} \partial_{\zeta}^{l} \pi_{\theta}\right| \\
& \lesssim\left\langle\xi^{\prime}\right\rangle^{1 / 2-|\alpha|-l} .
\end{aligned}
$$

Here $\equiv$ means equality modulo terms that satisfy the estimate. According to Theorem 3.7, there exists a parametrix to $S_{\theta}$ with symbol $s_{\theta}^{-\#} \in S_{1,1 / 2}^{0}\left(\mathbb{R}^{n-1} \times \mathbb{R}^{n}\right)$. We still need to show that multiplication by $\varphi_{1}$ reduces the order. As $\pi_{\theta}$ is elliptic, there exists a parametrix $\pi_{\theta}^{-\#}$ such that $\pi_{\theta} \pi_{\theta}^{-\#}-1=r_{\theta}^{\prime}$ is regularizing, and we find that

$$
\varphi_{1}=s_{\theta} \# \pi_{\theta}^{-\#}-\varphi_{1} \# r_{\theta}^{\prime}-\varphi_{0} \# \pi_{\theta}^{-\#} .
$$

Composition with $\varphi_{1}$ or $\varphi_{0}$ from the left is just pointwise multiplication. Hence we obtain the improved order of $s_{\theta}^{-\#} \# \varphi_{1}$ from the identities

$$
\begin{aligned}
& s_{\theta}^{-\#} \# \varphi_{1} \equiv s_{\theta}^{-\#} \#\left[s_{\theta} \# \pi_{\theta}^{-\#}-\varphi_{0} \pi_{\theta}^{-\#}\right] \quad \bmod S^{-\infty}\left(\mathbb{R}^{n-1} \times \mathbb{R}^{n}\right) \text { and } \\
& \equiv \pi_{\theta}^{-\#}-s_{\theta}^{-\#} \# \varphi_{0} \pi_{\theta}^{-\#} \quad \bmod S^{-\infty}\left(\mathbb{R}^{n-1} \times \mathbb{R}^{n}\right) .
\end{aligned}
$$


As $\varphi_{0} \pi_{\theta}^{-\#}, \pi_{\theta}^{-\#} \in S_{1,0}^{-1}$ and $s_{\theta}^{-\#} \in S_{1,1 / 2}^{0}$, this completes the proof.

\section{Bounded $\boldsymbol{H}^{\infty}$-calculus}

In this section we will prove Theorem 1.3.

\subsection{The half space and constant coefficients}

First, we consider the case where the underlying manifold is the euclidean half-space, the coefficients of the differential operator are constant and only the top order terms are non-zero. In symbols, $X=\mathbb{R}_{+}^{n}, a^{i j}(x)=a^{i j} \in \mathbb{R}, b^{j}(x)=0$ and $c^{0}(x)=0$. According to the last section, the resolvent of $A_{T}+v$ has the following structure:

$$
\left(A_{T}+v-\lambda\right)^{-1}=Q_{\theta, \mu,+}^{\prime}+G_{\theta, \mu}^{\prime}+R(\lambda),
$$

where $R(\lambda) \in \mathcal{L}\left(L_{p}\left(\mathbb{R}_{+}^{n}\right)\right)$ and $\|R(\lambda)\|=\mathcal{O}\left(\langle\lambda\rangle^{-1-\varepsilon}\right)$ for some $\varepsilon>0$. For the proof of Theorem 1.3 it is sufficient to provide Estimate (1.3). According to the equation above, we may estimate the three terms on the right hand side separately. The estimate for the first term is well-known, in fact it is the same as in the non-degenerate case. Any operator whose norm in $\mathcal{L}\left(L_{p}\left(\mathbb{R}_{+}^{n}\right)\right)$ is $\mathcal{O}\left(\langle\lambda\rangle^{-1-\varepsilon}\right)$, for some $\varepsilon>0$, is integrable along the boundary of $\Sigma_{\theta}$ and therefore the estimate holds. To provide the estimate for the singular Green part we need the following.

Lemma 4.1 Let $\sigma \in S_{1,0}^{1}\left(\mathbb{R}^{n-1} \times \mathbb{R}^{n}\right)$ and $\operatorname{Re} \sigma\left(x^{\prime}, \xi^{\prime}, \zeta\right) \geq c\left|\xi^{\prime}, \zeta\right|$. Then the map

$$
\mathbb{R}_{+} \ni t \mapsto \exp \left(-\sigma\left(x^{\prime}, \xi^{\prime}, \zeta\right) t\right) \in S_{1,0}^{0}\left(\mathbb{R}^{n-1} \times \mathbb{R}^{n}\right)
$$

is uniformly bounded. In fact, we have a bound $C=C\left(|\sigma|_{*}, c\right)$ on the seminorms.

Proof Induction over $|\alpha|+|\beta|+l=N$ shows that $D_{\xi^{\prime}}^{\alpha} D_{x^{\prime}}^{\beta} D_{\zeta}^{l} \exp \left(-\sigma\left(x^{\prime}, \xi^{\prime}, \zeta\right) t\right)$ is a linear combination over all $k \leq N, \alpha_{1}+\cdots+\alpha_{k}=\alpha, \beta_{1}+\cdots+\beta_{k}=\beta$, and $l_{1}+\cdots+l_{k}=l$. The terms in the linear combination have the following structure:

$$
\left(D_{\xi^{\prime}}^{\alpha_{1}} D_{x^{\prime}}^{\beta_{1}} D_{\zeta}^{l_{1}} \sigma\left(x^{\prime}, \xi^{\prime}, \zeta\right) \cdots D_{\xi^{\prime}}^{\alpha_{k}} D_{x^{\prime}}^{\beta_{k}} D_{\zeta}^{l_{k}} \sigma\left(x^{\prime}, \xi^{\prime}, \zeta\right)\right)(-t)^{k} \exp \left(-\sigma\left(x^{\prime}, \xi^{\prime}, \zeta\right) t\right)
$$

Furthermore, the assumption $\sigma \in S_{1,0}^{1}\left(\mathbb{R}^{n-1} \times \mathbb{R}^{n}\right)$ implies:

$$
\begin{aligned}
& \left|D_{\xi^{\prime}}^{\alpha_{1}} D_{x^{\prime}}^{\beta_{1}} D_{\zeta}^{l_{1}} \sigma\left(x^{\prime}, \xi^{\prime}, \zeta\right) \cdots D_{\xi^{\prime}}^{\alpha_{n}} D_{x^{\prime}}^{\beta_{k}} D_{\zeta}^{l_{k}} \sigma\left(x^{\prime}, \xi^{\prime}, \zeta\right)\right| \\
& \leq \prod_{i=1}^{k}|\sigma|_{*}\left|\xi^{\prime}, \zeta\right|^{1-\left|\alpha_{i}\right|-l_{i}}=|\sigma|_{*}^{k}\left|\xi^{\prime}, \zeta\right|^{k-|\alpha|-l} .
\end{aligned}
$$


Moreover, we use the fact that $s^{k} \exp (-s)$ is bounded on the positive real axis in order to obtain:

$$
\begin{aligned}
& \left|(-t)^{k} \exp \left(-\sigma\left(x^{\prime}, \xi^{\prime}, \zeta\right) t\right)\right| \\
& \quad=t^{k} \exp \left(-\operatorname{Re} \sigma\left(x^{\prime}, \xi^{\prime}, \zeta\right) t\right) \leq t^{k} \exp \left(-c\left|\xi^{\prime}, \zeta\right| t\right) \leq c^{-k}\left|\xi^{\prime}, \zeta\right|^{-k} C
\end{aligned}
$$

According to the last two estimates, all terms in the linear combination can be estimated by $C\left|\xi^{\prime}, \zeta\right|^{-|\alpha|-l}$.

Lemma 4.2 A constant $C=C\left(|t|_{*}, M, \vartheta\right)$ exists such that

$$
\left\|\int_{\partial \Sigma_{\theta}} f(\lambda) G_{\lambda}^{\prime} d \lambda\right\|_{\mathcal{B}\left(L_{p}\left(\mathbb{R}_{+}^{n}\right)\right)} \leq C\|f\|_{L_{\infty}\left(\Sigma_{\vartheta}\right)} \text { for all } f \in H_{0}^{\infty}\left(\Sigma_{\vartheta}\right) .
$$

Proof The boundary of $\Sigma_{\theta}$ consists of the two rays $e^{ \pm i \theta} \mathbb{R}$, which can be treated separately and analogously. Thus, providing the estimate for the following operator is sufficient:

$$
I^{+}:=2^{-1} e^{-i \theta} \int_{\lambda=e^{i \theta} \mu^{2}} f(\lambda) G_{\lambda}^{\prime} d \lambda=\int_{0}^{\infty} \mu f\left(\mu^{2} e^{i \theta}\right) G_{\theta, \mu}^{\prime} d \mu .
$$

For the estimate, we use the explicit description of the symbol-kernel of $G_{\theta}^{\prime}$ in Lemma 3.6. Since $s_{\theta}^{T} \in S_{1,1 / 2}^{-1}\left(\mathbb{R}^{n-1} \times \mathbb{R}^{n}\right), \zeta s_{\theta}^{T}\left(x^{\prime}, \xi^{\prime}, \zeta\right) \in S_{1,1 / 2}^{0}\left(\mathbb{R}^{n-1} \times \mathbb{R}^{n}\right)$. According to Remark 3.5, the $\kappa_{\theta}^{ \pm}$are strongly elliptic and a constant $c=c(M, \vartheta)>0$ exists such that:

$$
\operatorname{Re} \kappa_{\theta}^{ \pm}\left(x^{\prime}, \xi^{\prime}, \zeta\right) \geq 2 c|\xi, \zeta|
$$

Thus, $\sigma_{\theta}^{ \pm}\left(x^{\prime}, \xi^{\prime}, \zeta\right):=\kappa_{\theta}^{ \pm}\left(x^{\prime}, \xi^{\prime}, \zeta\right)-c \zeta$ satisfies the assumption of Lemma 4.1 and the map, below, is uniformly bounded:

$$
\begin{aligned}
& \mathbb{R}_{++}^{2} \ni\left(x_{n}, y_{n}\right) \mapsto h_{\theta}\left(x^{\prime}, \xi^{\prime}, \zeta ; x_{n}, y_{n}\right) \\
& \quad:=\zeta e^{c \zeta\left(x_{n}+y_{n}\right)} \tilde{g}_{\theta}^{\prime}\left(x^{\prime}, \xi^{\prime}, \zeta ; x_{n}, y_{n}\right) \in S_{1,1 / 2}^{0}\left(\mathbb{R}^{n-1} \times \mathbb{R}^{n}\right) .
\end{aligned}
$$

Now, we analyze the action of $G_{\theta, \mu}^{\prime}$ in the direction normal to the boundary. To this end, we define a family of operators that act on $\mathcal{S}\left(\mathbb{R}^{n-1}\right)$ :

$$
\left[G_{\theta, \mu}^{\prime}\left(x_{n}, y_{n}\right) v\right]\left(x^{\prime}\right):=\int e^{i x^{\prime} \xi^{\prime}} \tilde{g}_{\theta, \mu}^{\prime}\left(x^{\prime}, \xi^{\prime} ; x_{n}, y_{n}\right) \hat{v}\left(\xi^{\prime}\right) d \xi^{\prime}
$$

Correspondingly, we define $H_{\theta, \mu}\left(x_{n}, y_{n}\right)$ from $h_{\theta}$. Please note that:

$$
\mu e^{c \mu\left(x_{n}+y_{n}\right)} G_{\theta, \mu}^{\prime}\left(x_{n}, y_{n}\right)=H_{\theta, \mu}\left(x_{n}, y_{n}\right) .
$$


Since the seminorms of $h_{\theta}$ are uniformly bounded with respect to $\left(x_{n}, y_{n}\right) \in \mathbb{R}_{++}^{2}$, Theorem 3.2 shows that:

$$
\begin{aligned}
\left\|\mu G_{\theta, \mu}^{\prime}\left(x_{n}, y_{n}\right) v\right\|_{L_{p}\left(\mathbb{R}^{n-1}\right)} & \leq e^{-c \mu\left(x_{n}+y_{n}\right)}\left\|H_{\theta, \mu} v\right\|_{L_{p}\left(\mathbb{R}^{n-1}\right)} \\
& \leq e^{-c \mu\left(x_{n}+y_{n}\right)} C\|v\|_{L_{p}\left(\mathbb{R}^{n-1}\right)}
\end{aligned}
$$

Furthermore, if $u=v \otimes w \in \mathcal{S}\left(\mathbb{R}^{n-1}\right) \otimes \mathcal{S}\left(\mathbb{R}_{+}\right)$is a simple tensor, then:

$$
\left[I^{+} u\right]\left(x^{\prime}, x_{n}\right)=\int_{0}^{\infty} \int_{0}^{\infty} f\left(\mu^{2} e^{i \theta}\right)\left[\mu G_{\theta, \mu}^{\prime}\left(x_{n}, y_{n}\right) v\right]\left(x^{\prime}\right) w\left(y_{n}\right) d y_{n} d \mu .
$$

In order to provide the estimate for $I^{+}$, it is sufficient to consider simple tensors because they span a dense subset of $L_{p}\left(\mathbb{R}_{+}^{n}\right)$. Therefore:

$$
\begin{aligned}
& \left\|I^{+} u\right\|_{L_{p}\left(\mathbb{R}_{+}^{n}\right)} \leq\|f\|_{\infty}\left\|\int_{0}^{\infty} \int_{0}^{\infty}\right\| \mu G_{\theta, \mu}\left(x_{n}, y_{n}\right) v\left\|_{L_{p}\left(\mathbb{R}^{n-1}\right)}\left|w\left(y_{n}\right)\right| d y_{n} d \mu\right\|_{L_{p}\left(\mathbb{R}_{+}\right)} \\
& \quad \leq C\|f\|_{\infty}\|v\|_{L_{p}\left(\mathbb{R}^{n-1}\right)}\left\|\int_{0}^{\infty} \int_{0}^{\infty} \exp \left(-c \mu\left(x_{n}+y_{n}\right)\right)\left|w\left(y_{n}\right)\right| d y_{n} d \mu\right\|_{L_{p}\left(\mathbb{R}_{+}\right)} \\
& \quad \leq C\|f\|_{\infty}\|v\|_{L_{p}\left(\mathbb{R}^{n-1}\right)}\left\|\int_{0}^{\infty} \frac{\left|w\left(y_{n}\right)\right|}{x_{n}+y_{n}} d y_{n}\right\|_{L_{p}\left(\mathbb{R}_{+}\right)} \\
& \quad \leq C\|f\|_{\infty}\|v\|_{L_{p}\left(\mathbb{R}^{n-1}\right)}\|w\|_{L_{p}\left(\mathbb{R}_{+}\right)}=C\|f\|_{\infty}\|u\|_{L_{p}\left(\mathbb{R}_{+}^{n}\right)},
\end{aligned}
$$

where we used $L_{p}$-boundedness of the Hilbert transform for the latter inequality. The estimate implies that $I^{+} \in \mathcal{B}\left(L_{p}\left(\mathbb{R}_{+}^{n}\right)\right)$ and $\left\|I^{+}\right\| \leq C\|f\|_{L_{\infty}\left(\Sigma_{\vartheta}\right)}$. Here, $C=$ $C\left(M,|t|_{*}, \vartheta\right)$ is the constant in the estimate above.

We now have proven Theorem 1.3 for diffperential operator with constant coefficients.

Remark 4.3 The above arguments also provide the result for the case of smooth coefficients. However, in this case the constants also depend on the symbol seminorms of the differential operator.

\subsection{The Euclidean half space}

Now, we treat the situation where $X=\mathbb{R}_{+}^{n}$, but the coefficients of the differential operator may not be constant. We assume that $a^{i j} \in C^{\tau}\left(\mathbb{R}_{+}^{n}\right)$ for some $\tau>0$ and $b^{j}, c^{0} \in L_{\infty}\left(\mathbb{R}_{+}^{n}\right)$. We use the classical approach of freezing coefficients. We only freeze the coefficients of the differential operator, not those of the boundary operator. We use a localization scheme similar to that used by Kunstmann and Weis in [27]. This provides a family of operators that are small perturbations of an operator with frozen coefficients. We will prove that they allow a bounded $H^{\infty}$-calculus in a uniform manner. By patching together these operators, we can conclude that $A_{T}$ itself allows a bounded $H^{\infty}$-calculus. We choose a small $r>0$, how small we have to chose $r$ 
will become clear later on. We define the cubes $Q=(-r, r)^{n}$ and $Q_{l}:=Q+l$, with $l \in \Gamma:=r\left(\mathbb{Z} \times \mathbb{N}_{0}\right)$. Observe that $\mathbb{R}_{+}^{n} \subset \cup_{l \in \Gamma} Q_{l}$. We fix a positive function $\psi \in C_{c}^{\infty}(Q)$ such that $\gamma_{1} \psi=0$ and

$$
\sum_{l \in \Gamma} \psi_{l}(x)=1 \text { for all } x \in \mathbb{R}_{+}^{n} \text {, where } \psi_{l}(x)=\psi(x-l)
$$

Moreover, we choose a cut-off function $\chi \in C_{c}^{\infty}(Q)$ such that $\chi=1$ on supp $\psi$ and define $\chi_{l}(x):=\chi(x-l)$. We define $A_{l}$ as the $L_{p}$-realization with respect to the boundary operator $T$ of the following differential operator.

$$
\mathcal{A}_{l}=\mathcal{A}_{l}^{c}+\mathcal{A}_{l}^{s}=\sum_{|\alpha|=2} a_{\alpha}(l) D^{\alpha}+\sum_{|\alpha|=2} \chi_{l}(x)\left[a_{\alpha}(x)-a_{\alpha}(l)\right] D^{\alpha}
$$

Observe that $A_{l} \psi_{l}=A_{T}^{\prime} \psi_{l}$, where $A_{T}^{\prime}$ denotes the $L_{p}$-realization of the principal part of $\mathcal{A}$. The major technical difficulty is to show that each operator in the family $\left(A_{l}\right)_{l \in \Gamma}$ allows a bounded $H^{\infty}$-calculus, with uniform estimates. More precisely, for suitably chosen $r>0$ :

Lemma 4.4 The operator $A_{l}$ belongs to $H^{\infty}\left(\Sigma_{\theta}\right)$ for all $\theta>0$ and $l \in \Gamma$. Moreover there exists a $C:=C\left(M, \theta,\left\|a_{\alpha}\right\|_{C^{\tau}},|t|_{*}\right)>0$ such that

$$
\left\|f\left(A_{l}\right)\right\|_{\mathcal{B}\left(L_{p}\left(\mathbb{R}_{+}^{n}\right)\right)} \leq C\|f\|_{\infty} \text { for all } f \in H^{\infty}\left(\Sigma_{\theta}\right) \text { and } l \in \Gamma \text {. }
$$

We can choose $r>0$ such that $A_{l}^{s}$ is a small perturbation of $A_{l}^{c}+v$, in the sense of the following result. Recall that the shift $v$ was introduced to ensure the existence of a unique solution to the boundary problem.

Theorem 4.5 Let $E$ be a Banach space with the $U M D$ property, let $A \in \mathcal{S}(E)$ have a bounded $H^{\infty}\left(\Sigma_{\vartheta}\right)$-calculus, and $0 \in \rho(A)$. Suppose that $B$ is a linear operator in E such that $\mathcal{D}(A) \subset \mathcal{D}(B)$ and

$$
\|B u\|_{E} \leq \varepsilon\|A u\|_{E} \text { for all } u \in \mathcal{D}(A),
$$

for some $\varepsilon>0$. Suppose further that $\gamma \in(0,1)$ and a constant $C>0$ exist such that

$$
B\left(\mathcal{D}\left(A^{1+\gamma}\right)\right) \subset \mathcal{D}\left(A^{\gamma}\right) \text { and }\left\|A^{\gamma} B x\right\|_{E} \leq C\left\|A^{1+\gamma} x\right\|_{E} \text { for } x \in \mathcal{D}\left(A^{1+\gamma}\right)
$$

Then $A+B$ has a bounded $H^{\infty}\left(\Sigma_{\vartheta}\right)$-calculus in $E$, provided $\varepsilon$ is sufficiently small. Moreover, a constant $C_{A+B}=C_{A+B}\left(C_{A}, \varepsilon, C\right)$ exists such that

$$
\|f(A+B)\|_{\mathcal{B}(E)} \leq C_{A+B}\|f\|_{\infty} .
$$

For the proof we refer to [12]. To verify the assumptions of the theorem above, we observe: 
Lemma 4.6 A constant $C>0$ exists such that for $a_{l, \alpha}^{s}:=\chi_{l}\left(a_{\alpha}-a_{\alpha}(l)\right)$ :

$$
\left\|a_{l, \alpha}^{s}\right\|_{\infty} \leq C\left\|a_{\alpha}\right\|_{C^{\tau}\left(\mathbb{R}_{+}^{n}\right)} r^{\tau} \text { and }\left\|a_{l, \alpha}^{s}\right\|_{C^{\sigma}\left(\mathbb{R}_{+}^{n}\right)} \leq C\left\|a_{\alpha}\right\|_{C^{\tau}\left(\mathbb{R}_{+}^{n}\right)} r^{\tau-\sigma}
$$

given that $0<\sigma \leq \tau$.

Proof We recall that $r$ is proportional to the diameter of the cube $Q$. Thus,

$$
\begin{aligned}
\left\|a_{l, \alpha}^{s}\right\|_{\infty} & \leq \sup \left\{\frac{\left|a_{\alpha}(x)-a_{\alpha}(l)\right|}{|x-l|^{\tau}}|x-l|^{\tau}: x \in \operatorname{supp}\left(\chi_{l}\right)\right\} \\
& \leq C\left\|a_{\alpha}\right\|_{C^{\tau}\left(\mathbb{R}_{+}^{n}\right)} r^{\tau} .
\end{aligned}
$$

By a similar argument, we obtain the second estimate.

Next, we verify that the lemma above implies the following estimate:

$$
\left\|A_{l}^{S} u\right\|_{L_{p}\left(\mathbb{R}_{+}^{n}\right)} \leq C r^{\tau}\left\|\left(A_{l}^{c}+v\right) u\right\|_{L_{p}\left(\mathbb{R}_{+}^{n}\right)} \text { for all } u \in H_{p}^{2}\left(\mathbb{R}_{+}^{n}\right) \cap \operatorname{ker} T
$$

It is well-known that $C^{\tau}\left(\mathbb{R}_{+}^{n}\right) \hookrightarrow \mathcal{B}\left(H_{p}^{s}\left(\mathbb{R}_{+}^{n}\right)\right)$ as a multiplication operator for $0 \leq$ $s<\tau$. Therefore, with $s=0$ we obtain:

$$
\left\|A_{l}^{s} u\right\|_{L_{p}\left(\mathbb{R}_{+}^{n}\right)} \leq \sum_{1 \leq i, j \leq n}\left\|a_{l, i j}^{s}\right\|_{C\left(\mathbb{R}_{+}^{n}\right)}\|u\|_{H_{p}^{2}\left(\mathbb{R}_{+}^{n}\right)} \leq C r^{\tau}\|u\|_{H_{p}^{2}\left(\mathbb{R}_{+}^{n}\right)}
$$

Furthermore, on $H_{p}^{2}\left(\mathbb{R}_{+}^{n}\right) \cap \operatorname{ker} T$, the norm $\left\|\left(A_{l}^{c}+v\right) \cdot\right\|_{L_{p}\left(\mathbb{R}_{+}^{n}\right)}$ and the $H_{p}^{2}\left(\mathbb{R}_{+}^{n}\right)$ norm are equivalent because $\left(A_{l}^{c}+v\right)$ is invertible. Hence, Eq. (4.2) holds. Now, we compute the domain of $\left(A_{l}^{c}+v\right)^{\gamma}$ for $2 \gamma<\min \{1 / p, \tau\}$. According to Theorem [47, Theorem 1.15.2], the domain is:

$$
\mathcal{D}\left(\left(A_{l}^{c}+v\right)^{\gamma}\right)=\left(L_{p}\left(\mathbb{R}_{+}^{n}\right), H_{p}^{2}\left(\mathbb{R}_{+}^{n}\right) \cap \operatorname{ker} T\right)_{\gamma}
$$

We write $\dot{H}_{p}^{2}\left(\mathbb{R}_{+}^{n}\right)$ for the closure of $C_{c}^{\infty}\left(\mathbb{R}_{+}^{n}\right)$ in $H_{p}^{2}\left(\mathbb{R}_{+}^{n}\right)$. By interpolation, the embed$\operatorname{ding} \dot{H}_{p}^{2}\left(\mathbb{R}_{+}^{n}\right) \hookrightarrow H_{p}^{2}\left(\mathbb{R}_{+}^{n}\right) \cap \operatorname{ker} T \hookrightarrow H_{p}^{2}\left(\mathbb{R}_{+}^{n}\right)$ implies:

$$
\dot{H}_{p}^{2 \gamma}\left(\mathbb{R}_{+}^{n}\right) \hookrightarrow\left(L_{p}\left(\mathbb{R}_{+}^{n}\right), H_{p}^{2}\left(\mathbb{R}_{+}^{n}\right) \cap \operatorname{ker} T\right)_{\gamma} \hookrightarrow H^{2 \gamma}\left(\mathbb{R}_{+}^{n}\right)
$$

As $H_{p}^{2 \gamma}\left(\mathbb{R}_{+}^{n}\right)=\dot{H}_{p}^{2 \gamma}\left(\mathbb{R}_{+}^{n}\right)$ for $2 \gamma<1 / p$, we conclude that $\mathcal{D}\left(\left(A_{l}^{c}+\nu\right)^{\gamma}\right)=$ $H_{p}^{2 \gamma}\left(\mathbb{R}_{+}^{n}\right)$. Furthermore, the operator $\left(A_{l}^{c}+v\right)^{\gamma}$ is invertible. Thus, $\left\|\left(A_{l}^{c}+v\right)^{\gamma} \cdot\right\|_{L_{p}\left(\mathbb{R}_{+}^{n}\right)}$ and $\|\cdot\|_{H_{p}^{2 \gamma}\left(\mathbb{R}_{+}^{n}\right)}$ are equivalent norms on $\mathcal{D}\left(\left(A_{l}^{c}+v\right)^{\gamma}\right)$. We make use of Lemma 4.6 and the embedding $C^{\sigma}\left(\mathbb{R}_{+}^{n}\right) \hookrightarrow \mathcal{B}\left(H_{p}^{s}\left(\mathbb{R}_{+}^{n}\right)\right)$ to obtain the following estimate:

$$
\left\|\left(A_{l}^{c}+v\right)^{\gamma} A_{l}^{S} u\right\|_{L_{p}\left(\mathbb{R}_{+}^{n}\right)} \leq C\left\|A_{l}^{S} u\right\|_{H_{p}^{2 \gamma}\left(\mathbb{R}_{+}^{n}\right)} \leq C r^{\tau-2 \gamma}\|u\|_{H_{p}^{2+2 \gamma}\left(\mathbb{R}_{+}^{n}\right)} .
$$


We can further estimate the right hand side with [26, p. 70]:

$$
\|u\|_{H_{p}^{2+2 \gamma}\left(\mathbb{R}_{+}^{n}\right)} \leq C\left\|\left(v+A_{l}^{c}\right) u\right\|_{H_{p}^{2 \gamma}\left(\mathbb{R}_{+}^{n}\right)} \leq\left\|\left(v+A_{l}^{c}\right)^{1+\gamma} u\right\|_{L_{p}\left(\mathbb{R}_{+}^{n}\right)} .
$$

In sum, the following estimate holds for all $u \in \mathcal{D}\left(\left(\nu+A_{l}^{c}\right)^{1+\gamma}\right)$ :

$$
\left\|\left(v+A_{l}^{c}\right)^{\gamma} A_{l}^{s} u\right\|_{L_{p}\left(\mathbb{R}_{+}^{n}\right)} \leq C r^{\tau-2 \gamma}\left\|\left(\nu+A_{l}^{c}\right)^{1+\gamma} u\right\|_{L_{p}\left(\mathbb{R}_{+}^{n}\right)} .
$$

The constants in Eqs. (4.2) and (4.3) are independent of $l$ and $r$. Therefore, we can choose $r$ such that Theorem 4.5 applies to $v+A_{l}^{c}+A_{l}^{s}$ and thus Lemma 4.4 holds. Now we describe the localization scheme. We define $\mathbb{H}_{p}^{s}\left(\mathbb{R}_{+}^{n}\right):=l_{p}\left(\Gamma, H_{p}^{s}\left(\mathbb{R}_{+}^{n}\right)\right)$ and we write $\mathbb{L}_{p}\left(\mathbb{R}_{+}^{n}\right)$ if $s=0$. We introduce the localization operator $L$ and the patching operator $P$ with the help of a partition of unity (4.1):

$$
\begin{aligned}
& L: L_{p}\left(\mathbb{R}_{+}^{n}\right) \rightarrow \mathbb{L}_{p}\left(\mathbb{R}_{+}^{n}\right), \quad u \mapsto\left(\psi_{l} u\right)_{l \in \Gamma} . \\
& P: \mathbb{L}_{p}\left(\mathbb{R}_{+}^{n}\right) \rightarrow L_{p}\left(\mathbb{R}_{+}^{n}\right), \quad\left(u_{l}\right)_{l \in \Gamma} \mapsto \sum_{l \in \Gamma} \chi_{l} u_{l} .
\end{aligned}
$$

We also define the operator $\mathbb{T}: \mathbb{H}_{p}^{2}\left(\mathbb{R}_{+}^{n}\right) \rightarrow l_{p}\left(\Gamma ; B_{p}^{1-1 / p}\left(\mathbb{R}_{+}^{n}\right)\right),\left(u_{l}\right)_{l \in \Gamma} \rightarrow$ $\left(T u_{l}\right)_{l \in \Gamma}$. We collect some properties of these operators, which follow directly form the definitions:

Lemma 4.7 Let L, $P$ and $\mathbb{T}$ be as above. Then

(1) $L \in \mathcal{B}\left(H_{p}^{s}\left(\mathbb{R}_{+}^{n}\right) ; \mathbb{H}_{p}^{s}\left(\mathbb{R}_{+}^{n}\right)\right)$

(2) $P \in \mathcal{B}\left(\mathbb{H}_{p}^{s}\left(\mathbb{R}_{+}^{n}\right) ; H_{p}^{s}\left(\mathbb{R}_{+}^{n}\right)\right)$

(3) $P L=1$

(4) $L: H_{p}^{2}\left(\mathbb{R}_{+}^{n}\right) \cap \operatorname{ker} T \rightarrow \mathbb{H}_{p}^{2} \cap \operatorname{ker} \mathbb{T}$

(5) $P: \mathbb{H}_{p}^{2} \cap \operatorname{ker} \mathbb{T} \rightarrow H_{p}^{2}\left(\mathbb{R}_{+}^{n}\right) \cap \operatorname{ker} T$

We write $A_{l k}:=\delta_{l k} A_{l}$, with domain $\mathcal{D}\left(A_{l k}\right)=H_{p}^{2}\left(\mathbb{R}_{+}^{n}\right) \cap \operatorname{ker} T$. We define

$\mathbb{A}: \mathcal{D}(\mathbb{A}):=\mathbb{H}_{p}^{2}\left(\mathbb{R}_{+}^{n}\right) \cap \operatorname{ker} \mathbb{T} \subset \mathbb{L}_{p}\left(\mathbb{R}_{+}^{n}\right) \rightarrow \mathbb{L}_{p}\left(\mathbb{R}_{+}^{n}\right),\left(u_{k}\right)_{k \in \Gamma} \mapsto\left(\sum_{k \in \Gamma} A_{l k} u_{k}\right)_{l \in \Gamma}$

Similar we define $\mathbb{B}$ and $\mathbb{D}$ for the following families of operators.

$$
B_{l k}:=\delta_{l k} A_{l o w}+\left[\psi_{l}, A\right] \psi_{k} \text { and } D_{l k}=\delta_{l k} A_{l o w}+\psi_{l}\left[A_{k}+A_{l o w}, \psi_{k}\right]
$$

Here $A_{\text {low }}$ denotes the $L_{p}$-realisation with respect to the boundary operator $T$ of $\mathcal{A}-\mathcal{A}^{\prime}$. All sums in (4.4) are finite. In fact, we have a symmetric relation $l \bowtie k: \Leftrightarrow$ $\operatorname{supp} \psi_{l} \cap \operatorname{supp} \psi_{k} \neq \varnothing$ on $\Gamma$. The definition of $\psi_{l}$ implies that for fixed $l \in \Gamma$ the 
set $\Gamma_{l}:=\{k \in \Gamma: k \bowtie l\}$ is finite. Obviously $B_{l k}=0$ and $D_{l k}=0$ if $k \neq \Gamma_{l}$. The operators above are defined such that they satisfy the following relations.

$$
\begin{aligned}
& L A=(\mathbb{A}+\mathbb{B}) L \text { on } \mathcal{D}(A) \text { and } \\
& A P=P(\mathbb{A}+\mathbb{D}) \text { on } \mathcal{D}(\mathbb{A}) .
\end{aligned}
$$

For suitably chosen $r>0$ we obtain:

Lemma 4.8 The operator $\mathbb{A}$ belongs to $H^{\infty}\left(\Sigma_{\theta}\right)$ for each $\theta>0$.

Proof We fix $\theta>0$ and choose $r>0$ such that Lemma 4.4 applies. In particular, $\Sigma_{\theta} \subset \rho\left(A_{l}\right)$ for all $l \in \Gamma$ with uniform bounds on the inverse. Therefore, the inverse of $\lambda-\mathbb{A}$ exists and is given by $(\lambda-\mathbb{A})^{-1}\left(u_{l}\right)_{l \in \Gamma}=\left(\left(\lambda-A_{l}\right)^{-1} u_{l}\right)_{l \in \Gamma}$. For each $l \in \Gamma$ we have a bounded operator

$$
\hat{l}: \mathbb{L}_{p}\left(\mathbb{R}_{+}^{n}\right) \rightarrow L_{p}\left(\mathbb{R}_{+}^{n}\right), \quad\left(u_{k}\right)_{k \in \Gamma} \rightarrow u_{l} .
$$

Let $C$ be as in Lemma 4.4 and $f \in H_{*}^{\infty}\left(\Sigma_{\theta}\right)$. Then

$$
\begin{aligned}
\left\|f(\mathbb{A})\left(u_{k}\right)_{k \in \Gamma}\right\|_{\mathbb{L}_{p}\left(\mathbb{R}_{+}^{n}\right)}^{p} & =\sum_{l \in \Gamma}\left\|\hat{l} \int_{\partial \Sigma_{\theta}} f(\lambda)(\lambda+\mathbb{A})^{-1}\left(u_{k}\right)_{k \in \Gamma} d \lambda\right\|_{L_{p}\left(\mathbb{R}_{+}^{n}\right)}^{p} \\
& =\sum_{l \in \Gamma}\left\|\int_{\partial \Sigma_{\theta}} f(\lambda)\left(\lambda-A_{l}\right)^{-1} u_{l} d \lambda\right\|_{L_{p}\left(\mathbb{R}_{+}^{n}\right)}^{p} \\
& \leq \sum_{l \in \Gamma} C^{p}\|f\|_{\infty}^{p}\left\|u_{l}\right\|_{L_{p}\left(\mathbb{R}_{+}^{n}\right)}=C^{p}\|f\|_{\infty}^{p}\|u\|_{\mathbb{L}_{p}\left(\mathbb{R}_{+}^{n}\right)}^{p}
\end{aligned}
$$

This estimate is sufficient to see that $\mathbb{A} \in H^{\infty}\left(\Sigma_{\theta}\right)$.

Next, we observe that both $\mathbb{B}$ and $\mathbb{D}$ are lower order perturbations of $\mathbb{A}$ in the sense of the following well-known perturbation theorem going back to Amann. For a proof we refer to [27, Proposition 13.1].

Theorem 4.9 Let $A \in \mathcal{S}(E)$ have a bounded $H^{\infty}\left(\Sigma_{\theta}\right)$-calculus in $E$ and assume $0 \in \rho(A)$. Let $\gamma \in(0,1)$ and suppose that $B$ is a linear operator in $E$ satisfying $\mathcal{D}(B) \supset \mathcal{D}(A)$, and

$$
\|B u\|_{E} \leq C\left\|A^{1-\gamma} u\right\|_{E} \text { for all } u \in \mathcal{D}(A)
$$

where $C>0$. Then $v+A+B$ has a bounded $H^{\infty}\left(\Sigma_{\theta}\right)$-calculus in $E$ for $v \geq 0$ sufficiently large.

In particular, for suitably chosen $r>0$ we obtain: 
Lemma 4.10 For each $\theta>0$ a constant $v \geq 0$ exists such that both $v+\mathbb{A}+\mathbb{B}$ and $v+\mathbb{A}+\mathbb{D}$ belong to $H^{\infty}\left(\Sigma_{\theta}\right)$.

Proof We can assume that $0 \in \rho(\mathbb{A})$, otherwise we consider $v+\mathbb{A}$. Thus, $\mathbb{A}^{(1-\gamma)}$ is invertible and $\|\cdot\|_{\mathcal{D}\left(\mathbb{A}^{1-\gamma}\right)}$ is equivalent to $\left\|\mathbb{A}^{1-\gamma} \cdot\right\|_{\mathbb{L}_{p}\left(\mathbb{R}_{+}^{n}\right)}$. According to Lemma 4.8, the operator $\mathbb{A}$ belongs to $H^{\infty}\left(\Sigma_{\theta}\right)$ and therefore has bounded imaginary powers. According to [47, Theorem 1.15.2], the domain of $\mathbb{A}^{1-\gamma}$ is given by complex interpolation.

$$
\left.\left.\mathcal{D}\left(\mathbb{A}^{1-\gamma}\right)=\left(\mathbb{L}_{p}\left(\mathbb{R}_{+}^{n}\right), \mathcal{D}(\mathbb{A})\right)_{1-\gamma} \hookrightarrow\left(\mathbb{L}_{p}\left(\mathbb{R}_{+}^{n}\right), \mathbb{H}_{p}^{2}\left(\mathbb{R}_{+}^{n}\right)\right)\right)_{1-\gamma}=\mathbb{H}_{p}^{2-2 \gamma}\left(\mathbb{R}_{+}^{n}\right)\right)
$$

We can focus on $\mathbb{B}$, because the arguments for $\mathbb{D}$ are the same. A closer look on the definition of $B_{l k}$ reveals that it is a first order differential operator. In particular, for each $\gamma<1 / 2$ we have the standard estimate:

$$
\left\|B_{l k} u\right\|_{L_{p}\left(\mathbb{R}_{+}^{n}\right)} \leq C\|u\|_{H_{p}^{1}\left(\mathbb{R}_{+}^{n}\right)} \leq C\|u\|_{H_{p}^{2-2 \gamma}\left(\mathbb{R}_{+}^{n}\right)}
$$

Note that the constant $C>0$ only depends on the $L_{\infty}$-norm of the coefficients and thus can be chosen independent of $k$ and $l$. We write $N:=\sup _{l \in \Gamma} \#\{k \in \Gamma: k \bowtie l\}$. Then by estimate (4.7)

$$
\begin{aligned}
\left\|\mathbb{B}\left(u_{k}\right)_{k \in \Gamma}\right\|_{\mathbb{L}_{p}(\Gamma)}^{p} & =\sum_{l \in \Gamma}\left\|\sum_{k \bowtie l} B_{l k} u_{k}\right\|_{L_{p}\left(\mathbb{R}_{+}^{n}\right)}^{p} \leq \sum_{l \in \Gamma}\left(\sum_{k \bowtie l} C\left\|u_{k}\right\|_{H_{p}^{2-2 \gamma}\left(\mathbb{R}_{+}^{n}\right)}\right)^{p} \\
& \leq \sum_{l \in \Gamma} C^{p} N^{p} \sup _{k \bowtie l}\left\|u_{k}\right\|_{H_{p}^{2-2 \gamma}\left(\mathbb{R}_{+}^{n}\right)}^{p} \leq C^{p} N^{p} \sum_{l \in \Gamma} \sum_{k \bowtie l}\left\|u_{k}\right\|_{H_{p}^{2-2 \gamma}\left(\mathbb{R}_{+}^{n}\right)}^{p} \\
& \leq C^{p} N^{p+1} \sum_{l \in \Gamma}\left\|u_{l}\right\|_{H_{p}^{2-2 \gamma}\left(\mathbb{R}_{+}^{n}\right)}^{p}=C^{p} N^{p+1}\left\|\left(u_{l}\right)_{l \in \Gamma}\right\|_{\mathbb{H}^{2-2 \gamma}\left(\mathbb{R}_{+}^{n}\right)} \\
& \leq C^{p} N^{p+1}\left\|\mathbb{A}^{1-\gamma}\left(u_{l}\right)_{l \in \Gamma}\right\|_{\mathbb{L}_{p}\left(\mathbb{R}_{+}^{n}\right)} .
\end{aligned}
$$

In the fourth inequality we used the symmetry of the relation $\bowtie$ to change the order of summation. We finish the proof by the application of Theorem 4.9 to $v+\mathbb{A}+\mathbb{B}$.

Now, we can prove Theorem 1.3 for the case $X=\mathbb{R}_{+}^{n}$.

Proof For given $\theta>0$ we choose $v, r>0$ such that Lemma 4.10 applies. For each $\lambda \in \Sigma_{\theta}$ the operator $\lambda-\left(\nu+A_{T}\right)$ is invertible with left inverse $P(\lambda-(\nu+\mathbb{A}+\mathbb{B}))^{-1} L$ and right inverse $P(\lambda-(v+\mathbb{A}+\mathbb{D}))^{-1} L$. For all $f \in H_{*}^{\infty}\left(\Sigma_{\theta}\right)$ we have

$$
\left\|f\left(v+A_{T}\right)\right\| \leq\|P\|\|f(v+\mathbb{A}+\mathbb{B})\|\|L\| \leq C\|f\|_{\infty} .
$$

Therefore, $v+A_{T}$ allows a bounded $H^{\infty}\left(\Sigma_{\theta}\right)$-calculus. 


\subsection{Manifolds}

Now, let $(X, g)$ be a manifold with boundary and bounded geometry as in [17], see also [34]. We choose an atlas of Fermi coordinates $\kappa_{l}: U_{l} \subset X \rightarrow V_{l} \subset \overline{\mathbb{R}}_{+}^{n}$ with index set $\Gamma$ such that $\sup _{l \in \Gamma}\left|\left\{k \in \Gamma: U_{k} \cap U_{l} \neq \emptyset\right\}\right|=: N<\infty$. We also choose a subordinate partition of unity $\left(\psi_{l}\right)_{l \in \Gamma}$ such that $\partial_{\nu} \psi_{l}=0$ for all $l \in \Gamma$. Here, $v$ denotes an outward unit normal vector field on $\partial X$. For each $\psi_{l}$, we choose positive functions $\chi_{l}^{\prime}, \chi_{l} \in C_{c}^{\infty}\left(U_{l}\right)$ such that $\chi_{l}=1$ on supp $\psi_{l}$ and $\chi_{l}^{\prime}=1$ on supp $\chi_{l}$. We denote $\chi_{l, *}=\kappa_{l, *} \chi_{l} \in C_{c}^{\infty}\left(V_{l}\right) \subset C_{c}^{\infty}\left(\overline{\mathbb{R}}_{+}^{n}\right)$. Similarly, we define $\chi_{l, *}^{\prime}$. Moreover, we write $\tilde{\kappa}_{l}\left(x^{\prime}\right):=\kappa_{l}\left(x^{\prime}, 0\right)$ for the induced chart on the boundary. Let $\mathcal{A}$ be a sufficiently regular $M$-elliptic second order differential operator on $X$ as in (1.1) and $T$ be a boundary operator as in (1.2). For each $l \in \Gamma$, we define the following operators:

$$
\mathcal{A}_{l}:=-\Delta\left(1-\chi_{l, *}^{\prime}\right)+\kappa_{l, *} \mathcal{A} \kappa_{l}^{*} \chi_{l, *}^{\prime} \quad \text { and } \quad T_{l}:=\gamma_{0}\left(1-\chi_{l, *}^{\prime}\right)+\tilde{\kappa}_{l, *} T \kappa_{l}^{*} \chi_{l, *}^{\prime} .
$$

Then $\mathcal{A}_{l}$ is an $M$-elliptic second order differential operator on euclidean space with sufficiently regular coefficients. Moreover, the norms of the coefficients of the local representations of $\mathcal{A}$ are bounded by $M$. Therefore, the norms of the coefficients of $\mathcal{A}_{l}$ are uniformly bounded with respect to $l \in \Gamma$ and so are the seminorms $\left|t_{l}\right|_{*}$. We define:

$$
A_{l}: \mathcal{D}\left(A_{l}\right):=\left\{u \in H_{p}^{2}\left(\mathbb{R}_{+}^{n}\right): T_{l} u=0\right\} \rightarrow L_{p}\left(\mathbb{R}_{+}^{n}\right), u \mapsto r^{+} \mathcal{A}_{l} e^{+} u .
$$

Each operator $A_{l}$ satisfies the assumptions in the last subsection. Therefore, we can apply Theorem 1.3 to $A_{l}$, which implies that Lemma 4.4 continues to hold. We define the localization operator and the patching operator by:

$$
\begin{aligned}
& L: L_{p}(X) \rightarrow \mathbb{L}_{p}\left(\mathbb{R}_{+}^{n}\right), \quad u \mapsto\left(\kappa_{l, *} \psi_{l} u\right)_{l \in \mathbb{N}} . \\
& P: \mathbb{L}_{p}\left(\mathbb{R}_{+}^{n}\right) \rightarrow L_{p}(X), \quad\left(u_{l}\right)_{l \in \mathbb{N}} \rightarrow \sum_{l \in \mathcal{I}} \kappa_{l}^{*} \chi_{l, *} u_{l} .
\end{aligned}
$$

By definition, $u$ belongs to $H_{p}^{s}(X)$ if and only if $L u$ belongs to $\mathbb{H}_{p}^{s}\left(\mathbb{R}_{+}^{n}\right)$. Moreover, the norms of $u$ and $L u$ coincide. Therefore:

- $L \in \mathcal{B}\left(H_{p}^{s}(X) ; \mathbb{H}_{p}^{s}\left(\mathbb{R}_{+}^{n}\right)\right)$,

- $P \in \mathcal{B}\left(\mathbb{H}_{p}^{s}\left(\mathbb{R}_{+}^{n}\right) ; H_{p}^{s}(X)\right)$, and

- $P L=1$.

Remark 4.11 Spaces on manifolds with boundary and bounded geometry:

(a) It is natural to define $H_{p}^{s}(X)$ as $r^{+} H_{p}^{s}(\hat{X})$. Here $r^{+}$is the restriction in the sense of distributions, $H_{p}^{s}(\hat{X})=\left(I-\Delta_{g}\right)^{-s / 2} L_{p}(\hat{X})$ and $\hat{X}$ is a manifold with bounded geometry which contains $X$. For the existence of $\hat{X}$ we refer to [6]. The operator $\left(I-\Delta_{g}\right)^{-s / 2}$ is well defined for all $s \in \mathbb{R}$, due to the result of Strichartz in [40]. Since the restriction can be treated analogously to the euclidean or compact case, we may only consider $H_{p}^{s}(\hat{X})$. Let $L$ be defined as above with respect to an atlas 
of normal coordinates. Then $\|L \cdot\|_{\mathbb{H}_{p}^{s}\left(\mathbb{R}^{n}\right)}$ and $\left\|\left(I-\Delta_{g}\right)^{s} \cdot\right\|_{L_{p}(\hat{X})}$ are equivalent norms; this result is due to $\mathrm{H}$. Triebel, see [48, Theorem 7.4.5]. In [17], it was observed that an atlas of Fermi coordinates also gives rise to an equivalent norm.

(b) The interpolation results for $H_{p}^{s}\left(\mathbb{R}^{n}\right)$ extend to $H_{p}^{s}(\hat{X})$. This follows from two facts. First $H_{p}^{s}(\hat{X})$ is a retract of $\mathbb{H}_{p}^{s}\left(\mathbb{R}^{n}\right)$. Second $\mathbb{H}_{p}^{s}\left(\mathbb{R}^{n}\right)$ is the space of p-summable sequences with values in $H_{p}^{s}\left(\mathbb{R}^{n}\right)$.

(c) We may define Besov-spaces via real interpolation or via the localization operator $L$. According to part (b) both definitions coincide. The trace theorem holds on manifolds with boundary and bounded geometry, see [17] for the details.

Furthermore, we define $\mathbb{T}: \mathbb{H}_{p}^{2}\left(\mathbb{R}_{+}^{n}\right) \rightarrow \mathbb{B}_{p}^{1-1 / p}\left(\mathbb{R}^{n-1}\right),\left(u_{l}\right)_{l \in \mathcal{I}} \mapsto\left(T_{l} u_{l}\right)_{l \in \mathcal{I}}$. Using the fact $\partial_{\nu} \psi_{l}=0$ for all $l \in \Gamma$ we obtain: The localization operator maps the kernel of $T$ to the kernel of $\mathbb{T}$ and the patching operator maps the kernel of $\mathbb{T}$ into the kernel of $T$. We define $\mathcal{D}(\mathbb{A}):=\mathbb{H}_{p}^{2}\left(\mathbb{R}_{+}^{n}\right) \cap \operatorname{ker} \mathbb{T}$. Note that $\left(u_{l}\right)_{l \in \Gamma} \in \mathcal{D}(\mathbb{A})$ implies that $u_{l} \in \mathcal{D}\left(A_{l}\right)$ for all $l \in \Gamma$. Therefore, the following definition is reasonable:

$$
\mathbb{A}: \mathcal{D}(\mathbb{A}):=\mathbb{H}_{p}^{2}\left(\mathbb{R}_{+}^{n}\right) \cap \operatorname{ker} \mathbb{T} \subset \mathbb{L}_{p}\left(\mathbb{R}_{+}^{n}\right) \rightarrow \mathbb{L}_{p}\left(\mathbb{R}_{+}^{n}\right),\left(u_{l}\right)_{l \in \Gamma} \mapsto\left(A_{l} u_{l}\right)_{l \in \Gamma}
$$

Lemma 4.8 continues to hold as it only relies on Lemma 4.4. We define $\mathbb{B}, \mathbb{D}$ : $\mathbb{H}_{p}^{2}\left(\mathbb{R}_{+}^{n}\right) \subset \mathbb{L}_{p}\left(\mathbb{R}_{+}^{n}\right) \rightarrow \mathbb{L}_{p}\left(\mathbb{R}_{+}^{n}\right)$ as infinite matrices with entries:

$$
B_{l k}:=\kappa_{l_{*}}\left[\psi_{l}, A\right] \chi_{k, *}^{\prime} \kappa_{k}^{*} \quad \text { resp. } \quad D_{l k}:=\kappa_{l, *} \psi_{l} \kappa_{k}^{*}\left[A_{k}, \chi_{k, *}\right] .
$$

Again, the definition is motivated by the Relations (4.5) and (4.6). The operators $\mathbb{A}, \mathbb{B}, \mathbb{D}, L$ and $P$ have the same properties as those on the euclidean space. Therefore, the proof of Theorem 1.3 carries over.

\section{Non-smooth boundary operators}

In order to treat the case of non-smooth boundary operators, we may assume that $X=\mathbb{R}_{+}^{n}$ and $A$ has constant coefficients. The same perturbation arguments as before will then give the result for the general case. For the sake of simplicity we will moreover suppose that $\varphi_{0} \equiv 1$, and $\varphi_{1}=\varphi^{2}$, with $\varphi \in C^{2+\tau}$ and $\tau>0$. The operator $S_{\theta}=T K_{\theta}^{D}$ then is a pseudodifferential operator on the boundary with symbol:

$$
s_{\theta}(x, \xi, \zeta)=\varphi^{2}\left(x^{\prime}\right) \pi_{\theta}\left(\xi^{\prime}, \zeta\right)+1 \in C^{2+\tau} S_{1,0}^{1}\left(\mathbb{R}^{n-1} \times \mathbb{R}^{n}\right) .
$$

As in the smooth case, we associate an operator $S_{\lambda}$ to $S_{\theta}$ by restricting the additional covariable to $\mu$, where $\lambda=e^{ \pm \theta} \mu^{2}$. The following result is essential.

Proposition 5.1 For sufficiently large $|\lambda|$ and $s<\tau-1$, the operator $S_{\lambda}$ is invertible as an unbounded operator in $B_{p}^{s}\left(\mathbb{R}^{n-1}\right)$ with its maximal domain. Moreover,

$$
S_{\lambda}^{-1} \varphi^{2}: B_{p}^{s}\left(\mathbb{R}^{n-1}\right) \rightarrow B_{p}^{s+1}\left(\mathbb{R}^{n-1}\right) \text { is bounded, }
$$


and there exists an $s_{\theta}^{T} \in S_{1, \gamma}^{-1}\left(\mathbb{R}^{n-1} \times \mathbb{R}^{n}\right)$ and an $\varepsilon>0$, such that for $S_{\lambda}^{T}=\operatorname{op}\left(s_{\theta, \mu}^{T}\right)$ we have

$$
\left\|S_{\lambda}^{-1} \varphi^{2}-S_{\lambda}^{T}\right\|_{\mathcal{L}\left(L_{p}\left(\mathbb{R}^{n-1}\right)\right)} \leq C\langle\lambda\rangle^{-(1 / 2+\varepsilon)}
$$

The constant $C=C\left(M,\|\varphi\|_{C^{2+\tau}}\right)$ and the symbol seminorms of $s_{\theta}^{T}$ only depend on $M$ and $\|\varphi\|_{C^{2+\tau}}$.

Using the above result, the proof of Theorem 1.4 is analogous to that of Theorem 1.3, from Lemma 3.6 onwards.

Proof of Theorem 1.4 Recall that $\left(A_{\lambda,+}^{-1}+G_{\lambda}^{D}, K_{\lambda}^{D}\right)$ is the inverse to the Dirichlet problem and $G_{\lambda}^{T}=-K_{\lambda}^{D} S_{\lambda}^{-1} T\left(A_{\lambda,+}^{-1}+G_{\lambda}^{D}\right)$, see (1.9). In view of Eq. (5.2) and Theorem 3.2, the operator $A_{\lambda,+}^{-1}+G_{\lambda}^{D}+G_{\lambda}^{T}$ maps $L_{p}\left(\mathbb{R}_{+}^{n}\right)$ into $H_{p}^{2}\left(\mathbb{R}_{+}^{n}\right)$. Note that

$$
\begin{gathered}
A_{\lambda,+}\left(A_{\lambda,+}^{-1}+G_{\lambda}^{D}+G_{\lambda}^{T}\right)=1 \text { and } \\
T\left(A_{\lambda,+}^{-1}+G_{\lambda}^{D}+G_{\lambda}^{T}\right)=0 .
\end{gathered}
$$

Therefore, $A_{\lambda,+}^{-1}+G_{\lambda}^{D}+G_{\lambda}^{T}$ maps $L_{p}\left(\mathbb{R}_{+}^{n}\right)$ into the domain $\mathcal{D}\left(A_{T}\right)$ and is the resolvent. According to Eq. (5.3) and Theorem 3.2,

$$
\left\|K_{\lambda}^{D}\left(S_{\lambda}^{-1} \varphi^{2}-S_{\lambda}^{T}\right) \gamma_{1}\left(A_{\lambda,+}^{-1}+G_{\lambda}^{D}\right)\right\|_{\mathcal{L}\left(L_{p}\left(\mathbb{R}^{n}\right)\right)} \leq C\langle\lambda\rangle^{-(1+\varepsilon / 2)} .
$$

Thus, up to an error which is integrable with respect to $\lambda, G_{\lambda}^{T}$ coincides with

$$
G_{\lambda}^{T, *}=-K_{\lambda}^{D} S_{\lambda}^{T} \gamma_{1}\left(A_{\lambda,+}^{-1}+G_{\lambda}^{D}\right) .
$$

This operator has a symbol-kernel as in Lemma 3.6. The only difference is that now $\delta=\gamma$ instead of $\delta=1 / 2$, which does not affect the arguments given in Sect. 4 .

The rest of this section is dedicated to the proof of Proposition 5.1. Guided by the smooth case, we define a pseudodifferential operator on the boundary with symbol:

$$
s_{\theta}^{-\#}\left(x^{\prime}, \xi^{\prime}, \zeta\right):=\frac{1}{s_{\theta}\left(x^{\prime}, \xi^{\prime}, \zeta\right)}
$$

First we have to check that the function above is indeed a symbol. To this end we need the following estimates. 
Lemma 5.2 Let $s_{\theta}$ be as in (5.1). Then for all $\alpha, \beta \in \mathbb{N}^{n}$ with $|\beta| \leq 2$ a constant $C=C\left(M,\|\varphi\|_{C^{2+\tau}}\right)$ exists such that

$$
\begin{aligned}
&\left\|\frac{1}{s_{\theta}}\right\|_{\infty} \leq C, \\
&\left\|\frac{\partial_{\xi}^{\alpha} s_{\theta}}{s_{\theta}}\right\|_{\infty} \leq C\left\langle\xi^{\prime}, \zeta\right\rangle^{-|\alpha|}, \quad\left\|\frac{1}{s_{\theta}}\right\|_{C^{2+\tau}} \leq C\left\langle\xi^{\prime}, \zeta\right\rangle^{1+\tau / 2}, \\
&\left\|\frac{\partial_{x}^{\beta} s_{\theta}}{s_{\theta}}\right\|_{\infty} \leq C\left\langle\xi^{\prime}, \zeta\right\rangle^{|\beta| / 2} \text { and } \quad\left\|\frac{\partial_{\xi}^{\alpha} s_{\theta}}{s_{\theta}}\right\|_{C^{2+\tau}} \leq C\left\langle\xi^{\prime}, \zeta\right\rangle^{1+\tau / 2-|\alpha|} \\
& s_{\theta} \|_{C^{2+\tau-|\beta|}} \leq C\left\langle\xi^{\prime}, \zeta\right\rangle^{1+\tau / 2}
\end{aligned}
$$

Proof We first consider the Estimates (5.5). The function

$$
s_{\theta}^{-\#}=\frac{1}{s_{\theta}}=\frac{1}{\varphi^{2} \pi_{\theta}+1} .
$$

is bounded as $\operatorname{Re} \pi_{\theta}(\xi, \zeta) \geq c|\xi, \zeta| \geq 0$ with a constant $c=c(M)$. To estimate the Hölder norm we write

$$
s_{\theta}^{-\#}=f_{\pi_{\theta}^{1 / 2}}(\varphi), \text { with } f_{a}(s):=\frac{1}{(a s)^{2}+1} .
$$

By elementary calculus, we obtain

$$
\begin{aligned}
f_{a}^{\prime}(s) & =-2 a(a s) f_{a}^{2}(s) \\
f_{a}^{\prime \prime}(s) & =2 a^{2}\left(3(a s)^{2}-1\right) f_{a}^{3}(s) \text { and } \\
f_{a}^{\prime \prime \prime}(s) & =-24 a^{3}\left((a s)^{3}-(a s)\right) f_{a}^{4}(s)
\end{aligned}
$$

Note that, under the assumption that $a \in \Lambda_{\theta}$ for some $0<\theta<\pi / 2$, a constant $C=C(\theta)$ exists such that $\left|f_{a}(s)\right| \leq C$ and $\left|a s f_{a}(s)\right| \leq C$. Therefore, $\left\|f_{a}(s)\right\|_{C^{i}} \leq$ $C|a|^{i}$ for $i \in\{0,1,2,3\}$. By interpolation, we obtain $\left\|f_{a}(s)\right\|_{C^{2+\tau}} \leq C|a|^{2+\tau}$ for $0<\tau<1$. According to [23, Theorem A.8], the composition of $C^{2+\tau}$ functions is again a $C^{2+\tau}$ function, and

$$
\begin{aligned}
\left\|f_{\pi_{\theta}^{1 / 2}}(\varphi)\right\|_{C^{\tau}} & \leq C\left(\left\|f_{\pi_{\theta}^{1 / 2}}\right\|_{C^{2+\tau}}\|\varphi\|_{C^{1}}^{2+\tau}+\left\|f_{\pi_{\theta}^{1 / 2}}\right\|_{C^{1}}\|\varphi\|_{C^{2+\tau}}+\| f_{\pi_{\theta}^{1 / 2} \|_{\infty}}\right) \\
& \leq C\left(\left|\pi_{\theta}\right|^{1+\tau / 2}+\left|\pi_{\theta}\right|^{1 / 2}+1\right) \leq C\left\langle\xi^{\prime}, \zeta\right\rangle^{1+\tau / 2} .
\end{aligned}
$$

Here, the constant $C=C\left(M, \theta,\|\varphi\|_{C^{2+\tau}}\right)$ also depends on $\|\varphi\|_{C^{2+\tau}}$. Next, we consider the Estimates (5.6). For $\alpha \neq 0$

$$
\partial_{\xi}^{\alpha} s_{\theta}=\varphi^{2} \partial_{\xi}^{\alpha} \pi_{\theta}=\varphi^{2} \pi_{\theta} \frac{\partial_{\xi}^{\alpha} \pi_{\theta}}{\pi_{\theta}}
$$


As $\pi_{\theta} \in S_{1,0}^{1}\left(\mathbb{R}^{n-1} \times \mathbb{R}^{n}\right)$ is an elliptic symbol, the following estimates hold

$$
\left\|\frac{\partial_{\xi}^{\alpha} \pi_{\theta}}{\pi_{\theta}}\right\|_{\infty} \leq C\langle\xi\rangle^{-|\alpha|} \text { and }\left\|\frac{\partial_{\xi}^{\alpha} \pi_{\theta}}{\pi_{\theta}}\right\|_{C^{\tau}} \leq C\langle\xi\rangle^{-|\alpha|}
$$

We recall the well-known estimate for the Hölder norm of a product:

$$
\|g h\|_{C^{\sigma}} \leq\|g\|_{C^{\sigma}}\|h\|_{\infty}+\|g\|_{\infty}\|h\|_{C^{\sigma}}
$$

In view of Eqs. (5.8), (5.9) and (5.10), for the proof of (5.6) it is sufficient to prove:

$$
\left\|\frac{\varphi^{2} \pi_{\theta}}{s_{\theta}}\right\|_{\infty} \leq C \text { and }\left\|\frac{\varphi^{2} \pi_{\theta}}{s_{\theta}}\right\|_{C^{2+\tau}} \leq C\left\langle\xi^{\prime}, \zeta\right\rangle^{1+\tau / 2} .
$$

Using the fact that

$$
\frac{\varphi^{2} \pi_{\theta}}{s_{\theta}}=\frac{\varphi^{2} \pi_{\theta}}{\varphi^{2} \pi_{\theta}+1}=1-\frac{1}{\varphi^{2} \pi_{\theta}+1}=1-\frac{1}{s_{\theta}},
$$

these estimates follow from Estimates (5.5). Finally we consider Estimates (5.7). In case $|\beta|=2$, they follow from (5.5) and the fact that $s_{\theta} \in C^{2+\tau} S_{1,0}^{1}\left(\mathbb{R}^{n-1} \times \mathbb{R}^{n}\right)$. If $|\beta|=1$, we use the fact that $(a s) f_{a}(s)$ is bounded.

With these estimates at hand we can prove the following result.

Lemma 5.3 Let $s_{\theta}^{-\#}$ be as in (5.4). Then

$$
\begin{aligned}
s_{\theta}^{-\#} & \in C^{2+\tau} S_{1,1 / 2}^{0}\left(\mathbb{R}^{n-1} \times \mathbb{R}^{n}\right) \text { and } \\
\varphi^{2} s_{\theta}^{-\#} & \in C^{2+\tau} S_{1,1 / 2}^{-1}\left(\mathbb{R}^{n-1} \times \mathbb{R}^{n}\right)
\end{aligned}
$$

Proof According to Leibniz' rule, $\partial_{\xi}^{\alpha} s_{\theta}^{-\#}$ is a linear combination of terms, indexed by $k \leq|\alpha|$ and $\alpha_{1}+\cdots+\alpha_{k}=\alpha$, which have the following structure:

$$
\frac{1}{s_{\theta}} \frac{\partial_{\xi}^{\alpha_{1}} s_{\theta}}{s_{\theta}} \cdots \frac{\partial_{\xi}^{\alpha_{k}} s_{\theta}}{s_{\theta}}
$$

According to Lemma 5.2, the supremum norm of such a term is bounded by $C\left\langle\xi^{\prime}, \zeta\right\rangle^{-\left|\alpha_{i}\right|}$ and the Hölder norm is bounded by $C\left\langle\xi^{\prime}, \zeta\right\rangle^{1+\tau / 2-\left|\alpha_{i}\right|}$. Therefore, $s_{\theta}^{-\#}$ is a symbol of the claimed class. As $\varphi$ only depends on $x, \partial_{\xi}^{\alpha} \varphi^{2} s_{\theta}^{-\#}$ has a similar structure as $\partial_{\xi}^{\alpha} s_{\theta}^{-\#}$. We only have to replace the first factor by

$$
\frac{\varphi^{2}}{s_{\theta}}=\left(\frac{\varphi^{2} \pi_{\theta}}{s_{\theta}}\right) \pi_{\theta}^{-1}=\left(1-\frac{1}{s_{\theta}}\right) \pi_{\theta}^{-1} .
$$

Since $\pi_{\theta}$ is elliptic of order 1 , we have the claimed reduction in the order. 
In order to verify that $s_{\theta}^{-\#}$ is a coarse parametrix to $s_{\theta}$, we need some results on the composition of non-smooth pseudodifferential operators. In essence, we follow the argumentation in [1]. The key tool to handle the composition of non-smooth symbols is symbol smoothing:

Theorem 5.4 Let $p \in C_{*}^{\sigma} S_{1, \delta}^{m}\left(\mathbb{R}^{n-1} \times \mathbb{R}^{n}\right)$ and $\gamma \in(\delta, 1)$. Then symbols

$$
p^{\sharp} \in S_{1, \gamma}^{m}\left(\mathbb{R}^{n-1} \times \mathbb{R}^{n}\right) \text { and } p^{b} \in C_{*}^{\sigma} S_{1, \gamma}^{m-(\gamma-\delta) \sigma}\left(\mathbb{R}^{n-1} \times \mathbb{R}^{n}\right),
$$

exist such that $p=p^{\sharp}+p^{b}$. Moreover,

$$
\begin{array}{ll}
\partial_{x}^{\alpha} p^{\sharp} \in S^{m+\delta|\alpha|}\left(\mathbb{R}^{n-1} \times \mathbb{R}^{n}\right), & \text { if }|\alpha| \leq \sigma \text { and } \\
\partial_{x}^{\alpha} p^{\sharp} \in S^{m+\delta \sigma+\gamma(|\alpha|-\tau)}\left(\mathbb{R}^{n-1} \times \mathbb{R}^{n}\right), & \text { if }|\alpha|>\sigma .
\end{array}
$$

For the proof of the result, we refer to [46, §1.3].

Let $p \in C_{*}^{\sigma_{p}} S_{1, \delta_{p}}^{m_{p}}\left(\mathbb{R}^{n-1} \times \mathbb{R}^{n}\right)$ and $q \in C_{*}^{\sigma_{q}} S_{1, \delta_{q}}^{m_{q}}\left(\mathbb{R}^{n-1} \times \mathbb{R}^{n}\right)$. For all $k<\sigma_{q}$ we define the truncated composition:

$$
t_{k}(p, q):=\sum_{|\alpha| \leq k} \frac{1}{\alpha !} D_{\xi}^{\alpha} p \partial_{x}^{\alpha} q
$$

Moreover, we write $t_{k}^{b}(p, q)=t_{k}\left(p, q^{\mathrm{b}}\right)$ and $t_{k}^{\sharp}(p, q)=t_{k}\left(p, q^{\sharp}\right)$, where $q^{\sharp}$ and $q^{\mathrm{b}}$ are as in Theorem 5.4 with $\gamma \in\left(\delta_{q}, 1\right)$. Note that $t_{k}^{\sharp}$ is defined for all $k \in \mathbb{N}_{0}$. Clearly the truncated composition is a symbol, given as a sum over symbols of different order and regularity. In order to facilitate the analysis, we set $t_{-1}(p, q):=0$ and introduce the difference $d_{k}(p, q):=t_{k}(p, q)-t_{k-1}(p, q)$. Similarly we define $d_{k}^{\mathrm{b}}(p, q)$ and $d_{k}^{\sharp}(p, q)$. From the definition we conclude that:

$$
\begin{aligned}
& d_{k}(p, q) \in C_{*}^{\min \left(\sigma_{p}, \sigma_{q}-k\right)} S_{1, \max \left(\delta_{p}, \delta_{q}\right)}^{m_{p}+m_{q}-\left(1-\delta_{q}\right) k}\left(\mathbb{R}^{n-1} \times \mathbb{R}^{n}\right), \\
& d_{k}^{\mathrm{b}}(p, q) \in C_{*}^{\min \left(\sigma_{p}, \sigma_{q}-k\right)} S_{1, \max \left(\delta_{p}, \gamma\right)}^{m_{p}+m_{q}-\left(\gamma-\delta_{q}\right) \sigma_{q}-(1-\gamma) k}\left(\mathbb{R}^{n-1} \times \mathbb{R}^{n}\right), \\
& d_{k}^{\sharp}(p, q) \in C_{*}^{\sigma_{p}} S_{1, \max \left(\delta_{p}, \gamma\right)}^{m_{p}+m_{q}-\left(1-\delta_{q}\right) k}\left(\mathbb{R}^{n-1} \times \mathbb{R}^{n}\right), \quad \text { if } k \leq \sigma_{q} \text { and } \\
& d_{k}^{\sharp}(p, q) \in C_{*}^{\sigma_{p}} S_{1, \max \left(\delta_{p}, \gamma\right)}^{m_{p}+m_{q}-\left(1-\delta_{q}\right) \sigma_{q}-(1-\gamma)\left(k-\sigma_{q}\right)}\left(\mathbb{R}^{n-1} \times \mathbb{R}^{n}\right), \text { if } k>\sigma_{q} .
\end{aligned}
$$

To each symbol defined above we associate a pseudodifferential operator. We denote the operator by capital letters, e.g. $P:=p(x, D)$. To further simplify the notation we drop the dependence on $p$ and $q$ if these are obvious, e.g. $t_{k}=t_{k}(p, q)$ or $T_{k}=$ $t_{k}(x, D)=t_{k}(p, q)(x, D)$. 
We now analyze the composition. To this end we fix $n, n^{\prime} \in \mathbb{N}_{0}$, such that $n^{\prime} \leq n$ and $n^{\prime} \leq \sigma_{q}$. Then:

$$
\begin{aligned}
P Q & =P\left(Q^{\sharp}+Q^{b}\right)=P Q^{\sharp}+P Q^{b}=T_{n}^{\sharp}+\left(P Q^{\sharp}-T_{n}^{\sharp}\right)+P Q^{b} \\
& =T_{n^{\prime}}+\left(T_{n^{\prime}}^{\sharp}-T_{n^{\prime}}\right)+\left(T_{n}^{\sharp}-T_{n^{\prime}}^{\sharp}\right)+\left(P Q^{\sharp}-T_{n}^{\sharp}\right)+P Q^{b} .
\end{aligned}
$$

The first term on the right hand side is the truncated composition and the other terms are remainder terms, which we now analyze. We define $R_{n^{\prime}}:=T_{n^{\prime}}^{\sharp}-T_{n^{\prime}}, R_{n, n^{\prime}}:=T_{n}^{\sharp}-T_{n^{\prime}}^{\sharp}$, $R_{n}^{\sharp}:=P Q^{\sharp}-T_{n}^{\sharp}$, and $R^{\text {b }}:=P Q^{\text {b }}$. In view of Eq. (5.12), the term

$$
R_{n^{\prime}}=T_{n^{\prime}}^{\sharp}-T_{n^{\prime}}=-T_{n^{\prime}}^{b}
$$

is a pseudodifferential operator with symbol

$$
r_{n^{\prime}} \in C_{*}^{\min \left(\sigma_{p}, \sigma_{q}-n^{\prime}\right)} S_{\max \left(\delta_{p}, \gamma\right)}^{m_{p}+m_{q}-\left(\gamma-\delta_{q}\right) \sigma_{q}}\left(\mathbb{R}^{n-1} \times \mathbb{R}^{n}\right) .
$$

We point out that the following term is a pseudodifferential operator with the same regularity as $p$.

$$
R_{n^{\prime}, n}=\left(T_{n}^{\sharp}-T_{n^{\prime}}^{\sharp}\right)=\sum_{k=n^{\prime}+1}^{n} D_{k}^{\sharp} \text {. }
$$

To be precise, Eqs. (5.13) and (5.14) imply that the symbol belongs to

$$
r_{n^{\prime}, n} \in \begin{cases}C_{*}^{\sigma_{p}} S_{\max \left(\delta_{p}, \gamma\right)}^{m_{p}+m_{q}-\left(1-\delta_{q}\right)\left(n^{\prime}+1\right)} & \text { if } n^{\prime}+1 \leq \sigma_{q} \text { and } \\ C_{*}^{\sigma_{p}} S_{\max \left(\delta_{p}, \gamma\right)}^{m_{p}+m_{q}-\left(1-\delta_{q}\right) \sigma_{q}-(1-\gamma)\left(n^{\prime}+1-\sigma_{q}\right)} & \text { if } n^{\prime}+1>\sigma_{q} .\end{cases}
$$

We use the well-known fact that $P Q^{\sharp} \sim \sum_{k \in \mathbb{N}_{0}} D_{k}^{\sharp}$ in order to analyze

$$
R_{n}^{\sharp}=P Q^{\sharp}-T_{n}^{\sharp} \sim \sum_{k>n} D_{k}^{\sharp} .
$$

In view of Eqs. (5.13) and (5.14), the symbol of the operator above belongs to

$$
r_{n}^{\sharp} \in \begin{cases}C_{*}^{\sigma_{p}} S_{\max \left(\delta_{p}, \gamma\right)}^{m_{p}+m_{q}-\left(1-\delta_{q}\right)(n+1)} & \text { if } n+1 \leq \sigma_{q} \text { and } \\ C_{*}^{\sigma_{p}} S_{\max \left(\delta_{p}, \gamma\right)}^{m_{p}+m_{q}-\left(1-\delta_{q}\right) \sigma_{q}-(1-\gamma)\left(n+1-\sigma_{q}\right)} & \text { if } n+1>\sigma_{q} .\end{cases}
$$

The analysis of $R^{b}=P Q^{b}$ relies on the fact that $Q^{b}$ has lower order than $Q$.

We now apply the results above to the case of interest, which is $p=s_{\theta}$ and $q=s_{\theta}^{-\#}$. The parameters in this case are $m_{p}=1, m_{q}=0, \delta_{p}=0, \delta_{q}=1 / 2$ and 
$\sigma_{p}=\sigma_{q}=2+\tau$, with $\tau>0$. We fix $n^{\prime}=0, n=2$ and $\gamma \in(1 /(2+\tau)+1 / 2,1)$. We define $2 \varepsilon=\min ((\gamma-1 / 2)(2+\tau)-1,1)>0$. Then Eq. (5.12) implies

$$
d_{k}^{b} \in C_{*}^{2+\tau-k} S_{1, \gamma}^{-2 \varepsilon}\left(\mathbb{R}^{n-1} \times \mathbb{R}^{n}\right), \text { for } k \in\{0,1,2\} .
$$

In particular, since $R_{0}=D_{0}^{\mathrm{b}}$ we have

$$
R_{0}: B_{p}^{s-2 \varepsilon}\left(\mathbb{R}^{n}\right) \rightarrow B_{p}^{s}\left(\mathbb{R}^{n}\right) \text { for } 0 \leq s<2+\tau
$$

We make the following observation. According to Lemma 5.2:

$$
\begin{aligned}
& d_{0}=\frac{s_{\theta}}{s_{\theta}}=1, \\
& d_{1}=-\sum_{|\alpha|=1} \frac{D_{\xi}^{\alpha} s_{\theta}}{s_{\theta}} \frac{\partial_{x}^{\alpha} s_{\theta}}{s_{\theta}} \in C_{*}^{1+\tau} S_{1,1 / 2}^{-1 / 2}\left(\mathbb{R}^{n-1} \times \mathbb{R}^{n}\right) \text { and } \\
& d_{2}=-\sum_{\substack{|\alpha|=2, 0<\alpha^{\prime}<\alpha}} \frac{1}{\alpha !} \frac{D_{\xi}^{\alpha} s_{\theta}}{s_{\theta}}\left(\frac{\partial_{x}^{\alpha} s_{\theta}}{s_{\theta}}-2 \frac{\partial_{x}^{\alpha-\alpha^{\prime}} s_{\theta}}{s_{\theta}} \frac{\partial_{x}^{\alpha^{\prime}} s_{\theta}}{s_{\theta}}\right) \in C_{*}^{\tau} S_{1,1 / 2}^{-1}\left(\mathbb{R}^{n-1} \times \mathbb{R}^{n}\right) .
\end{aligned}
$$

Please note that these terms have lower order than the general theory implies. We may write $D_{k}^{\sharp}=D_{k}-D_{k}^{b}$ for $k \in\{1,2\}$. Then

$$
\begin{aligned}
& D_{1}^{\sharp}: B_{p}^{s-\varepsilon}\left(\mathbb{R}^{n}\right) \rightarrow B_{p}^{s}\left(\mathbb{R}^{n}\right), \quad \text { if } 0 \leq s<1+\tau \text { and } \\
& D_{2}^{\sharp}: B_{p}^{s-2 \varepsilon}\left(\mathbb{R}^{n}\right) \rightarrow B_{p}^{s}\left(\mathbb{R}^{n}\right), \quad \text { if } 0 \leq s<\tau .
\end{aligned}
$$

On the other hand, the general theory implies

$$
D_{2}^{\sharp}: B_{p}^{s}\left(\mathbb{R}^{n}\right) \rightarrow B_{p}^{s}\left(\mathbb{R}^{n}\right), \quad \text { if } 0 \leq s<2+\tau .
$$

By complex interpolation we obtain

$$
D_{2}^{\sharp}: B_{p}^{s-\varepsilon}\left(\mathbb{R}^{n}\right) \rightarrow B_{p}^{s}\left(\mathbb{R}^{n}\right), \text { if } 0 \leq s<1+\tau \text {. }
$$

In particular, as $R_{0,2}=D_{1}^{\sharp}+D_{2}^{\sharp}$ we have

$$
R_{0,2}: B_{p}^{s-\varepsilon}\left(\mathbb{R}^{n}\right) \rightarrow B_{p}^{s}\left(\mathbb{R}^{n}\right) \text { for } 0 \leq s<1+\tau
$$

According to Eq. (5.15), we have

$$
R_{2}^{\sharp}: B_{p}^{s-2 \varepsilon}\left(\mathbb{R}^{n}\right) \rightarrow B_{p}^{s}\left(\mathbb{R}^{n}\right) \text { for } 0 \leq s<2+\tau .
$$


Theorem 5.4 yields

$$
Q^{b}: B_{p}^{s-2 \varepsilon}\left(\mathbb{R}^{n}\right) \rightarrow B_{p}^{s+1}\left(\mathbb{R}^{n}\right) \text { if } 0 \leq s<1+\tau .
$$

Since $P: B_{p}^{s+1}\left(\mathbb{R}^{n}\right) \rightarrow B_{p}^{s}\left(\mathbb{R}^{n}\right)$ if $0 \leq s<2+\tau$, we obtain

$$
R^{b}: B_{p}^{s-2 \varepsilon}\left(\mathbb{R}^{n}\right) \rightarrow B_{p}^{s}\left(\mathbb{R}^{n}\right) \text { if } 0 \leq s<1+\tau
$$

We define $R_{\theta}^{R}:=S_{\theta} S_{\theta}^{-\#}-I$, then $R_{\theta}^{R}=R_{0}+R_{0,2}+R_{2}^{\sharp}+R^{b}$. Thus

$$
R_{\theta}^{R}: B_{p}^{s-\varepsilon}\left(\mathbb{R}^{n}\right) \rightarrow B_{p}^{s}\left(\mathbb{R}^{n}\right) \text { if } 0 \leq s<1+\tau .
$$

We also compute the left remainder. In this case $p=s_{\theta}^{-\#}$ and $q=s_{\theta}$, thus the parameters are $m_{p}=0, m_{q}=1, \delta_{p}=1 / 2, \delta_{q}=0$ and $\sigma_{p}=\sigma_{q}=2+\tau$. We fix $n^{\prime}=0, n=1$ and $\gamma=1 / 2$. Then $\left(\gamma-\delta_{q}\right)(2+\tau)=1+\tau / 2 \geq 1+2 \varepsilon$, with the same $\varepsilon$ as above. According to Eq. (5.12),

$$
d_{k}^{b} \in C_{*}^{2+\tau-k} S_{1,1 / 2}^{-2 \varepsilon-k / 2}\left(\mathbb{R}^{n-1} \times \mathbb{R}^{n}\right)
$$

In particular, as $R_{0}=D_{0}^{\mathrm{b}}$ we have

$$
R_{0}: B_{p}^{s-2 \varepsilon}\left(\mathbb{R}^{n}\right) \rightarrow B_{p}^{s}\left(\mathbb{R}^{n}\right), \text { for } 0 \leq s<2+\tau
$$

As before, we obtain:

$$
d_{0}=\frac{s_{\theta}}{s_{\theta}}=1 \text { and } d_{1}=-\sum_{|\alpha|=1} \frac{D_{\xi}^{\alpha} s_{\theta}}{s_{\theta}} \frac{\partial_{x}^{\alpha} s_{\theta}}{s_{\theta}} \in C_{*}^{1+\tau} S_{1,1 / 2}^{-1 / 2}\left(\mathbb{R}^{n-1} \times \mathbb{R}^{n}\right)
$$

Thus, if we write $D_{1}^{\sharp}=D_{1}-D_{1}^{b}$ we obtain

$$
D_{1}^{\sharp}: B_{p}^{s-1 / 2}\left(\mathbb{R}^{n}\right) \rightarrow B_{p}^{s}\left(\mathbb{R}^{n}\right), \text { if } 0 \leq s<1+\tau \text {. }
$$

Since $\delta_{q}=0$ in this case, the general theory implies

$$
D_{1}^{\sharp}: B_{p}^{s}\left(\mathbb{R}^{n}\right) \rightarrow B_{p}^{s}\left(\mathbb{R}^{n}\right), \text { if } 0 \leq s<2+\tau .
$$

Therefore, by complex interpolation with parameter $1-2 \varepsilon$ we obtain

$$
D_{1}^{\sharp}: B_{p}^{s-\varepsilon}\left(\mathbb{R}^{n}\right) \rightarrow B_{p}^{s}\left(\mathbb{R}^{n}\right) \text { if } 0 \leq s<2+\tau-\varepsilon .
$$


Since $R_{0,1}=D_{1}^{\sharp}$, the above result holds for $R_{0,1}$. We want to point out that $\tau-\varepsilon>0$. We choose $n=1$ in this case, as $r_{1}^{\sharp}$ already has order -1 . We therefore have

$$
R_{1}^{\sharp}: B_{p}^{s-\varepsilon}\left(\mathbb{R}^{n}\right) \rightarrow B_{p}^{s}\left(\mathbb{R}^{n}\right) \text { if } 0 \leq s<2+\tau .
$$

Note that $q^{b}$ has order $-2 \varepsilon$. Since $p$ has order zero, we obtain

$$
R^{b}: B_{p}^{s-\varepsilon}\left(\mathbb{R}^{n}\right) \rightarrow B_{p}^{s}\left(\mathbb{R}^{n}\right) \text { if } 0 \leq s<2+\tau .
$$

We define $R_{\theta}^{L}:=S_{\theta}^{-\#} S_{\theta}-I$, then $R_{\theta}^{L}=R_{0}+R_{1,0}+R_{1}^{\sharp}+R^{b}$. Therefore,

$$
R_{\theta}^{L}: B_{p}^{s-\varepsilon}\left(\mathbb{R}^{n}\right) \rightarrow B_{p}^{s}\left(\mathbb{R}^{n}\right) \text { if } 0 \leq s<2+\tau-\varepsilon
$$

Now we analyze the parameter dependence of the above operator. To this end we need to extend Theorem 3.2 to non-smooth symbols.

Theorem 5.5 Let $p \in C_{*}^{\sigma} S_{1, \delta}^{-m}\left(\mathbb{R}^{n-1} \times \mathbb{R}^{n}\right)$ and $m \geq 0$. Then

$$
P_{\mu} \in \mathcal{L}\left(L_{p}\left(\mathbb{R}^{n-1}\right) ; H_{p}^{s}\left(\mathbb{R}^{n-1}\right)\right) \text { and }\left\|P_{\mu}\right\| \leq C|p|_{*}\langle\mu\rangle^{-m+s} \text {, }
$$

for $0 \leq s \leq m$ and $s<\sigma(1-\delta)$.

Proof We fix $\gamma \in(\delta, 1)$ such that $s \gamma<\sigma(\gamma-\delta)$. According to Theorem 5.4 a decomposition $p=p^{\sharp}+p^{b}$ exists, with $p^{\sharp} \in S_{1, \gamma}^{-m}\left(\mathbb{R}^{n-1} \times \mathbb{R}^{n}\right)$ and $p^{b} \in$ $C_{*}^{\sigma} S_{1, \gamma}^{-m-\sigma(\gamma-\delta)}\left(\mathbb{R}^{n-1} \times \mathbb{R}^{n}\right)$. According to Theorem 3.2,

$$
P_{\mu}^{\sharp} \in \mathcal{L}\left(L_{p}\left(\mathbb{R}^{n-1}\right) ; H_{p}^{s}\left(\mathbb{R}^{n-1}\right)\right) \text { and }\left\|P_{\mu}^{\sharp}\right\| \leq C|p|_{*}\langle\mu\rangle^{-m+s} \text {. }
$$

Note that for any $s, s^{\prime} \leq 0$ the estimate $\langle\xi, \mu\rangle^{s+s^{\prime}} \leq\langle\xi\rangle^{s}\langle\mu\rangle^{s^{\prime}}$ holds. Therefore, the map

$$
C_{*}^{\sigma} S_{1, \gamma}^{-m-\sigma(\gamma-\delta)}\left(\mathbb{R}^{n-1} \times \mathbb{R}^{n}\right) \ni p \mapsto p_{\mu} \in C_{*}^{\sigma} S_{1, \gamma}^{-s}\left(\mathbb{R}^{n-1} \times \mathbb{R}^{n-1}\right)
$$

is bounded by $\langle\mu\rangle^{-m+s}$. The result follows from the well-known mapping properties of non-smooth pseudodifferential operators.

Corollary 5.6 The operators $R_{\lambda}^{R}$ and $R_{\lambda}^{L}$ belong to $\mathcal{L}\left(L_{p}\left(\mathbb{R}^{n-1}\right)\right)$ and their norms are bounded by $C\langle\lambda\rangle^{-\varepsilon / 2}$, with $C=C\left(M,\|\varphi\|_{C^{2+\tau}}\right)$.

Proof Theorem 5.5 directly applies to all symbols of the components of $R_{\theta}^{R}$ and $R_{\theta}^{L}$, except for $R_{\theta}^{b}=P_{\theta} Q_{\theta}^{b}$, where we have to use the theorem twice.

Proposition 5.7 For sufficiently large $|\lambda|, 1-R_{\lambda}^{R}$ and $1-R_{\lambda}^{L}$ are invertible in $\mathcal{L}\left(L_{p}\left(\mathbb{R}^{n-1}\right)\right)$. Moreover, $\left(1-R_{\lambda}^{R}\right)^{-1} \in \mathcal{L}\left(B_{p}^{s}\left(\mathbb{R}^{n-1}\right)\right)$ for $0 \leq s<1+\tau$ and $\left(1-R_{\lambda}^{L}\right)^{-1} \in \mathcal{L}\left(B_{p}^{s}\left(\mathbb{R}^{n-1}\right)\right)$ for $0 \leq s<2+\tau-\varepsilon$. 
Proof Let $R=R_{\lambda}^{R}$ or $R=R_{\lambda}^{L}$. According to Corollary 5.6, the $\mathcal{L}\left(L_{p}\left(\mathbb{R}^{n-1}\right)\right)$-norm of $R$ is less than 1 , if $|\lambda|$ is large. Therefore, the inverse is given by a Neumann series

$$
(1-R)^{-1}=\sum_{k \geq 0} R^{k}=\sum_{K-1 \geq k \geq 0} R^{k}+R^{K}(1-R)^{-1}
$$

The finite sum belongs to $\mathcal{L}\left(B_{p}^{s}\left(\mathbb{R}^{n-1}\right)\right)$, since $R$ does. We fix $K$ such that $K \varepsilon>s$. Since $H_{p}^{s^{\prime}}\left(\mathbb{R}^{n-1}\right) \hookrightarrow B_{p}^{s}\left(\mathbb{R}^{n-1}\right)$ for $s^{\prime}>s, R^{K} \in \mathcal{L}\left(L_{p}\left(\mathbb{R}^{n-1}\right) ; B_{p}^{s}\left(\mathbb{R}^{n-1}\right)\right)$. in view of the fact that $B_{p}^{s}\left(\mathbb{R}^{n-1}\right) \hookrightarrow L_{p}\left(\mathbb{R}^{n-1}\right)$ for $s>0$, we obtain $R^{K}(1-R)^{-1} \in$ $\mathcal{L}\left(B_{p}^{s}\left(\mathbb{R}^{n-1}\right)\right)$.

Now we can prove Proposition 5.1.

Proof By definition, we have $S_{\lambda} S_{\lambda}^{-\#}=1-R_{\lambda}^{L}$ and $S_{\lambda}^{-\#} S_{\lambda}=1-R_{\lambda}^{R}$. According to Proposition 5.7, for sufficiently large $|\lambda|$, the left and right inverse to $S_{\lambda}$ exist and belong to $\mathcal{L}\left(B_{p}^{s}\left(\mathbb{R}^{n-1}\right)\right)$, if $0 \leq s<1+\tau$. The inverse is

$$
S_{\lambda}^{-1}=S_{\lambda}^{-\#}\left(1-R_{\lambda}^{L}\right)^{-1}=\left(1-R_{\lambda}^{R}\right)^{-1} S_{\lambda}^{-\#}
$$

Now we consider $S_{\lambda}^{-1} \varphi^{2}=\left(1-R_{\lambda}^{R}\right)^{-1} S_{\lambda}^{-\#} \varphi^{2}$. In view of Lemma 5.3, $\varphi^{2} S_{\lambda}^{-\#}$ maps $B_{p}^{s}\left(\mathbb{R}^{n-1}\right)$ to $B_{p}^{s+1}\left(\mathbb{R}^{n-1}\right)$ for $s<1+\tau$. Moreover, $\left(1-R_{\lambda}^{R}\right)^{-1} \in \mathcal{L}\left(B_{p}^{s}\left(\mathbb{R}^{n-1}\right)\right)$ for $s<2+\tau-\varepsilon$, according to Proposition 5.7. Thus, it is sufficient to consider the commutator $\left[S_{\lambda}^{-\#}, \varphi^{2}\right]$. To this end, we apply the results on the composition to $p=s_{\theta}^{-\#} \in C^{2+\tau} S_{1,1 / 2}^{0}$ and $q=\varphi^{2} \in C^{2+\tau} S_{1,0}^{0}$, we fix $n^{\prime}=0, n=1$ and $\gamma<1$ such that $(2+\tau)(\gamma-1 / 2)>1$. Then

$$
\begin{aligned}
{\left[S_{\theta}^{-\#}, \varphi^{2}\right] } & =R_{0}+R_{0,1}+R_{1}^{\sharp}+R^{b}, \text { with : } \\
r_{0} & \in C^{2+\tau} S_{1, \gamma}^{-(2+\tau)(\gamma-1 / 2)}\left(\mathbb{R}^{n-1} \times \mathbb{R}^{n}\right), \\
r_{0,1} & \in S_{1, \gamma}^{-1}\left(\mathbb{R}^{n-1} \times \mathbb{R}^{n}\right), \\
r_{1}^{\sharp} & \in S_{1, \gamma}^{-2}\left(\mathbb{R}^{n-1} \times \mathbb{R}^{n}\right), \\
q^{b} & \in C^{2+\tau} S_{1, \gamma}^{-\tau(\gamma-1 / 2)}\left(\mathbb{R}^{n-1} \times \mathbb{R}^{n}\right)
\end{aligned}
$$

Thus, $\left[S_{\lambda}^{-\#}, \varphi^{2}\right.$ ] belongs to $\mathcal{L}\left(B_{p}^{s}\left(\mathbb{R}^{n-1}\right) ; B_{p}^{s+1}\left(\mathbb{R}^{n-1}\right)\right)$ for $0 \leq s<1+\tau$ and Eq. (5.2) holds. We observe, that $r_{0,1}=d_{1}^{\sharp}=d_{1}-d_{1}^{b} \in C^{1+\tau} S_{1, \gamma}^{-1-1 / 2}$, since:

$$
d_{1}=\sum_{|\alpha|=1} D_{\xi}^{\alpha} s^{-\#} \partial_{x}^{\alpha} \varphi^{2}=\sum_{|\alpha|=1} \frac{D_{\xi}^{\alpha} s_{\theta}}{s_{\theta}} \frac{\partial_{x}^{\alpha} s_{\theta}}{s_{\theta}} \pi_{\theta}^{-1} \in C^{1+\tau} S_{1,1 / 2}^{-1-1 / 2}\left(\mathbb{R}^{n-1} \times \mathbb{R}^{n}\right) .
$$


According to Theorem 5.5, the operator $\left[S_{\lambda}^{-\#}, \varphi^{2}\right] \in \mathcal{L}\left(L_{p}\left(\mathbb{R}^{n-1}\right)\right)$ and $\left\|\left[S_{\lambda}^{-\#}, \varphi^{2}\right]\right\| \in$ $\mathcal{O}\left(\langle\lambda\rangle^{-(1+\varepsilon) / 2}\right)$. Thus

$$
\begin{aligned}
& S_{\lambda}^{-1} \varphi^{2}-\varphi^{2} S_{\lambda}^{-\#}=\left[S^{-\#}, \varphi^{2}\right]+R_{\lambda}^{R}\left(1-R_{\lambda}^{R}\right)^{-1} S_{\lambda}^{-\#} \in \mathcal{L}\left(L_{p}\left(\mathbb{R}^{n-1}\right)\right) \text { and } \\
& \left\|S_{\lambda}^{-1} \varphi^{2}-\varphi^{2} S_{\lambda}^{-\#}\right\| \in \mathcal{O}\left(\langle\lambda\rangle^{-(1+\varepsilon) / 2}\right) .
\end{aligned}
$$

We denote by $s_{\theta}^{T}$ the smooth part of the symbol of $\varphi^{2} S_{\theta}^{-\#}$. According to Lemma 5.3 and Theorem 5.4, $s_{\theta}^{T} \in S_{1, \gamma}^{-1}\left(\mathbb{R}^{n-1} \times \mathbb{R}^{n}\right)$ and $\varphi^{2} s_{\theta}^{-\#}-s_{\theta}^{T} \in S_{1, \gamma}^{-(1+\varepsilon)}\left(\mathbb{R}^{n-1} \times \mathbb{R}^{n}\right)$. In view of Theorem 5.5, we have $\varphi^{2} S_{\lambda}^{-\#}-S_{\lambda}^{T} \in \mathcal{L}\left(L_{p}\left(\mathbb{R}^{n-1}\right)\right)$ and $\left\|\varphi^{2} S_{\lambda}^{-\#}-S_{\lambda}^{T}\right\| \in$ $\mathcal{O}\left(\langle\lambda\rangle^{-(1+\varepsilon) / 2}\right)$.

\section{The porous medium equation}

In this section, we illustrate the applicability of the theory developed so far to nonlinear parabolic partial differential equations. A prominent example for this type of equations is the porous medium Eq. (1.6). It arises for instance in the description of the gas flow through a porous medium. As pointed out, we consider the case where the initial value $v_{0} \in H_{p}^{2}(X)$ satisfies $v_{0} \geq c$ for some $c>0$ and the boundary value $\phi$ is in $C^{1}\left(J_{0} ; B_{p, T}^{1-1 / p}(\partial X)\right), J_{0}=\left[0, t_{0}\right]$ and compatible with the initial value, i.e., $\phi(0)=T v_{0}$. Under this assumption, Theorem 1.9 provides the short time existence of a solution. We recall a result on short time existence for quasi-linear evolution equations.

\subsection{Quasi-linear evolution equations}

Let $1<q<\infty, E_{0}$ be a Banach space, $E_{1} \hookrightarrow E_{0}$ densely, $J_{0}=\left[0, t_{0}\right]$ for some $t_{0}>0$. We consider the quasi-linear problem

$$
\dot{u}(t)+A(t, u(t)) u(t)=F(t, u(t)), \quad t \in J_{0}, u(0)=0 .
$$

Here $E_{q}:=\left(E_{0}, E_{1}\right)_{1-1 / q, q}, A: J_{0} \times E_{q} \rightarrow \mathcal{L}\left(E_{1}, E_{0}\right)$ is continuous, and $F: J_{0} \times$ $E_{q} \rightarrow E_{0}$ satisfies assumptions of the Caratheodory type, i.e. $F(\cdot, u)$ is measurable for each $u \in E_{q}, F(t, \cdot)$ is continuous for a.a. $t \in J_{0}$, and $f(\cdot):=F(\cdot, 0) \in L_{q}\left(J_{0} ; E_{0}\right)$. Moreover, we assume the following condition on local Lipschitz continuity of $A$ and $F$ for some $R^{*}>0$ :

(A) For each $R \in\left(0, R^{*}\right)$ there is a constant $C=C(R)$ such that

$$
\|A(t, u) v-A(t, \bar{u}) v\|_{E_{0}} \leq C\|u-\bar{u}\|_{E_{q}}\|v\|_{E_{1}}, \quad t \in J_{0}, u, \bar{u} \in B(0, R), v \in E_{1} .
$$

(F) For each $R \in\left(0, R^{*}\right)$ there is a function $\psi_{R} \in L_{q}\left(J_{0}\right)$ such that

$$
\|F(t, u)-F(t, \bar{u})\|_{E_{0}} \leq \psi_{R}(t)\|u-\bar{u}\|_{E_{q}}, \quad \text { a.a. } t \in J_{0}, u, \bar{u} \in B(0, R) .
$$


Theorem 6.1 Suppose assumptions $(\boldsymbol{A})$ and $(\boldsymbol{F})$ are satisfied, and assume that $A_{0}=$ $A\left(0, u_{0}\right)$ has the property of maximal $L_{q}$-regularity. Then there is a $t^{*}>0$ such that (6.1) admits a unique solution $u$ on $J=\left[0, t^{*}\right]$ in the maximal regularity class $u \in H_{q}^{1}\left(J ; E_{0}\right) \cap L_{q}\left(J ; E_{1}\right)$

The result goes back to [10]. We use the formulation given in [31], except for the fact that we assume $u_{0}=0$ and require Lipschitz continuity of $A$ and $F$ only for some $R^{*}>0$ while in [31, Theorem 3.1] $R^{*}=\infty$ is assumed. The latter can be achieved easily by modifying $A$ and $F$ outside a neighborhood of zero:

Let $A$ satisfy assumption (A). Fix a positive function $\chi \in C^{\infty}\left(\mathbb{R}_{+}\right)$, which is 1 on $\left[0, R^{*} / 2\right]$ and 0 on $\left[R^{*}, \infty\right)$. We define

$$
\tilde{A}(t, u):=\chi\left(\|u\|_{E_{q}}\right) A(t, u)+\left(1-\chi\left(\|u\|_{E_{q}}\right)\right) A(t, 0) .
$$

Then $\tilde{A}$ satisfies Assumption (A), with $R^{*}=\infty$. Similarly

$$
\tilde{F}(t, u):=\chi\left(\|u\|_{E_{q}}\right) F(t, u)+\left(1-\chi\left(\|u\|_{E_{q}}\right)\right) F(t, 0) .
$$

satisfies Assumption (F), with $R^{*}=\infty$. By definition $\tilde{A}\left(0, u_{0}\right)=A\left(0, u_{0}\right)$ and therefore $\tilde{A}\left(0, u_{0}\right)$ has the property of maximal $L_{q}$-regularity, if $A\left(0, u_{0}\right)$ does. Under the assumptions of Theorem 6.1, [31, Theorem 3.1] implies that a unique solution $u \in H_{q}^{1}\left(J ; E_{0}\right) \cap L_{q}\left(J ; E_{1}\right)$ to

$$
\dot{u}+\tilde{A}(t, u)=\tilde{F}(t, u), \quad u(0)=u_{0} .
$$

exists. It is well-known that the solution belongs to $C\left(J ; E_{q}\right)$. Thus, for a possibly shorter $J$ we can assume that $u(t) \in B\left(u_{0}, R^{*} / 2\right)$ for all $t \in J$. Therefore, $\tilde{A}(t, u(t))=$ $A(t, u(t))$ and $\tilde{F}(t, u(t))=F(t, u(t))$ for all $t \in J$. Hence, $u$ solves Eq. (6.1).

\subsection{Proof of Theorem 1.9}

As a first step we reduce to the case of homogeneous boundary condition. To this end, we need the following result.

Lemma 6.2 Let $\phi \in C^{1}\left(J_{0} ; B_{p, T}^{1-1 / p}(\partial X)\right)$ and $v_{0} \in H_{p}^{2}(X)$. Then there exists $a$ $w \in C^{1}\left(J_{0} ; H_{p}^{2}(X)\right)$, such that $T w=\phi$ and $w(0)=v_{0}$.

Proof By Proposition 1.7, $T: H_{p}^{s}(X) \rightarrow B_{p, T}^{s}(\partial X)$ is linear, bounded and surjective. According to a result of Michael [30, Corollary, p. 364] every linear bounded surjective map between two Banach spaces has a continuous right inverse. Hence there exists a continuous lifting of the continuous map $\dot{\phi}: J_{0} \rightarrow B_{p, T}^{1-1 / p}$, i.e. a map $\tilde{w} \in C\left(J_{0} ; H_{p}^{2}(X)\right)$ such that $T \tilde{w}(t)=\dot{\phi}(t)$. We define

$$
w(t)=\int_{0}^{t} \tilde{w}(s) d s+v_{0} .
$$


Then $w \in C^{1}\left(J_{0} ; H_{p}^{s}(X)\right)$ and

$$
T w(t)=T \int_{0}^{t} \tilde{w}(s) d s+T v_{0}=\int_{0}^{t} \dot{\phi}(t)+\phi(0)=\phi(t) .
$$

Clearly $w(0)=v_{0}$.

We define $u:=v-w$ and consider the following parabolic problem:

$$
\left\{\begin{array}{l}
\dot{u}(t)-\Delta_{g}(u(t)+w(t))^{m}=-\dot{w}(t) \\
T u(t)=0 \\
u(0)=0 .
\end{array}\right.
$$

A quick computation shows that $v$ solves (1.6) if and only if $u$ solves (6.3). Therefore, we focus on Problem (6.3) which we rewrite as a quasi-linear evolution equation. To this end, we need the following identity which can easily be verified in local coordinates.

$$
\begin{aligned}
\Delta_{g}(u+w)^{m}= & m(u+w)^{m-1} \Delta_{g} u \\
& +m(m-1)(u+w)^{m-2}|\nabla(u+w)|_{g}^{2}+m((u+w))^{m-1} \Delta_{g} w .
\end{aligned}
$$

The first term on the right hand side is the highest order term. Therefore, we define

$$
\begin{aligned}
A(t, u(t)):= & -m(u(t)+w(t))^{m-1} \Delta_{g, T} \text { and } \\
F(t, u(t)):= & m(m-1)(u(t)+w(t))^{m-2}|\nabla(u(t)+w(t))|_{g}^{2} \\
& m((u(t)+w(t)))^{m-1} \Delta_{g, T} w(t)-\dot{w}(t) .
\end{aligned}
$$

According to the definitions above, Problem (6.3) is the quasi-linear evolution equation:

$$
\dot{u}(t)+A(t, u(t)) u(t)=F(t, u(t)), \quad u(0)=0 .
$$

In the following, we verify that Theorem 6.1 can be applied to (6.4), which proves Theorem 1.9. We define $E_{0}=L_{p}(X)$ and $E_{1}=H_{p}^{2}(X) \cap \operatorname{ker} T$. Then

$$
E_{q}=\left(E_{0}, E_{1}\right)_{1-1 / q, q} \hookrightarrow\left(L_{p}(X), H_{p}^{2}(X)\right)_{1-1 / q, q}=B_{p, q}^{2-2 / q}(X) \hookrightarrow C^{1}(X) .
$$

Here, the last embedding holds since $2-2 / q-n / p>1>0$ by assumption. Please note that $A(0, u(0))=m v_{0}^{m-1} \Delta_{g, T}$ satisfies the assumptions of Theorem 1.3 as $v_{0}$ is Hölder continuous and strictly positive. Therefore, a suitable shift of $A(0, u(0))$ allows a bounded $H^{\infty}$-calculus and thus $A(0, u(0))$ has maximal $L_{q}$-regularity. In order to verify the assumptions (A) and (F) we need the following result, which is inspired by [33]. 
Lemma 6.3 Let $w \in C\left(J_{0} ; C^{\tau}(X)\right)$ with $\operatorname{Re} w(0, \cdot) \geq c>0$. Let

$$
W:=\left\{z \in \mathbb{C}:|z|<\|w(0, \cdot)\|_{C^{\tau}}+3 c / 4, \quad \operatorname{Re} z>c / 4\right\} .
$$

Then an interval $J=\left[0, t^{*}\right]$, with $t^{*}>0$, a neighborhood $V$ of zero in $C^{\tau}(X)$, and a constant $C:=C\left(c,\|w(0, \cdot)\|_{C^{\tau}(X)}\right)$ exist such that for all $f \in H^{\infty}(W), t \in J$, and $u, \bar{u} \in V$ the following estimates hold:

$$
\begin{gathered}
\|f(u+w(t))\|_{C^{\tau}(X)} \leq C\|f\|_{L_{\infty}(W)} \text { and } \\
\|f(u+w(t))-f(\bar{u}+w(t))\|_{C^{\tau}(X)} \leq C\|f\|_{L_{\infty}(W)}\|u-\bar{u}\|_{C^{\tau}(X)} .
\end{gathered}
$$

Proof Since $w$ depends continuously on $t$, we can chose a $t^{*}>0$ such that for all $t \in J:=\left[0, t^{*}\right]$ we have $\|w(t)-w(0)\|_{C^{\tau}(X)} \leq c / 8$. We choose $V:=\left\{u \in C^{\tau}\right.$ : $\left.\|u\|_{C^{\tau}(X)}<c / 8\right\}$. As all functions in $V$ are continuous, we obtain for all $t \in J$ :

$$
\begin{aligned}
& \operatorname{im} V+w(t) \subset W^{\prime \prime}, \text { here } \operatorname{im} V:=\cup_{u \in V} \operatorname{im} u \text { and } \\
& W^{\prime \prime}:=\left\{z \in \mathbb{C}:|z|<\|w(0)\|_{C^{\tau}}+c / 4, \operatorname{Re} z>3 c / 4\right\} .
\end{aligned}
$$

Furthermore, we define

$$
W^{\prime}:=\left\{z \in \mathbb{C}:|z|<\|w(0)\|_{C^{\tau}}+c / 2, \quad \operatorname{Re} z>c / 2\right\} .
$$

By definition, some distance between the boundary of $W^{\prime \prime}$ and the boundary of $W^{\prime}$ exists, i.e., $d\left(\partial W^{\prime \prime}, \partial W^{\prime}\right) \geq c / 4$. Therefore, $|\eta-(u(x)+w(t, x))| \geq c / 4$ for all $t \in J, u \in V, \eta \in \partial W^{\prime}$ and $x \in X$. It is well-known that such a lower bound implies that $(\eta-(u(\cdot)+w(t, \cdot)))^{-1} \in C^{\tau}(X)$. Moreover, the following estimate holds:

$$
\begin{aligned}
\left\|(\eta-(u(\cdot)+w(t, \cdot)))^{-1}\right\|_{C^{\tau}(X)} & \leq 16 / c^{2}\|\eta-(u(\cdot)+w(t, \cdot))\|_{C^{\tau}(X)} \\
& \leq 16 / c^{2}\left(2\|w(0)\|_{C^{\tau}(x)}+c\right)=: S
\end{aligned}
$$

We can estimate the length of the boundary $\partial W^{\prime}$ :

$$
\left|\partial W^{\prime}\right| \leq 2 \pi\left(\|w(0)\|_{C^{\tau}(X)}+c / 2\right)=: 2 \pi L .
$$

For all $u \in V, t \in J$, and $x \in X$ we obtain the following identity from the Cauchy integral representation:

$$
f(u(x)+w(t, x))=\frac{1}{2 \pi i} \int_{\partial W^{\prime}} f(\eta)(\eta-(u(x)+w(t, x)))^{-1} d \eta .
$$

Thus, we obtain the first estimate $\|f(u+w(t))\|_{C^{\tau}(X)} \leq L S\|f\|_{H^{\infty}(W)}$. Now, let $u, \bar{u} \in V, t \in J, x \in X$, and use the resolvent identity to obtain:

$$
\begin{aligned}
& f(u(x)+w(t, x))-f(\bar{u}(x)+w(t, x))=(\bar{u}(x)-u(x)) I(t, x), \text { here } \\
& I(t, x):=\frac{1}{2 \pi i} \int_{\partial W^{\prime}} f(\eta)(\eta-(u(x)+w(t, x)))^{-1}(\eta-(\bar{u}(x)+w(t, x)))^{-1} d \eta
\end{aligned}
$$


We can estimate the $C^{\tau}(X)$-norm of $I(t, x)$ as before. Therefore, we obtain the second claimed estimate.

According to Lemma 6.2, $w \in C^{1}\left(J_{0} ; H_{p}^{2}(X)\right)$. Under the assumptions of Theorem 1.9, $H_{p}^{2}(X) \hookrightarrow C^{\tau}(X)$ and therefore $w \in C\left(J_{0} ; C^{\tau}(X)\right)$. We chose an interval $J$ and a neighborhood $V$ according to Lemma 6.3. Therefore, we can choose a neighborhood $U$ of zero in $E_{q}$ such that its image under the embedding belongs to $V$. We apply Lemma 6.3 to $f(z):=z^{m-j}$, with $j \in\{0,1\}$. Therefore, a constant exists such that for all $u, \bar{u} \in U$ and $t \in J$ :

$$
\begin{gathered}
\left\|(u+w(t))^{m-j}\right\|_{C^{\tau}(X)} \leq C \text { and } \\
\left\|(u+w(t))^{m-j}-(\bar{u}+w(t))^{m-j}\right\|_{C^{\tau}(X)} \leq C\|u-\bar{u}\|_{E_{q}} .
\end{gathered}
$$

In view of the embeddings $E_{q} \hookrightarrow C^{\tau}(X)$ and $C^{\tau}(X) \hookrightarrow \mathcal{L}\left(E_{0}\right)$, Eq. (6.5) implies that $A(t, u)$ satisfies Assumption (A):

$$
\begin{aligned}
\|A(t, u) v-A(t, \bar{u}) v\|_{E_{0}} & \leq m\left\|\left((u+w(t))^{m-1}-(\bar{u}+w(t))^{m-1}\right)\right\| \mathcal{L}_{\left(E_{0}\right)}\left\|\Delta_{g, T} v\right\|_{E_{0}} \\
& \leq C\|u-\bar{u}\|_{E_{q}}\|v\|_{E_{1}} .
\end{aligned}
$$

Now, we consider $F(t, u)$.

$$
\begin{aligned}
F(t, u)-F(t, \bar{u})= & m(m-1)(u(t)+w(t))^{m-1}|\nabla(u(t)+w(t))|^{2} \\
& -m(m-1)(\bar{u}(t)+w(t))^{m-1}|\nabla(\bar{u}(t)+w(t))|^{2} \\
& +m((u(t)+w(t)))^{m-1}-\left(\bar{u}(t)+w(t)^{m-1}\right) \Delta_{g, T} w(t) .
\end{aligned}
$$

Since $w \in C^{1}\left(J ; H_{p}^{2}(X)\right)$, the map $t \mapsto\left\|\Delta_{g} w(t, \cdot)\right\|_{E_{0}}$ belongs to $L_{q}(J)$. In view of Eq. (6.6), we obtain

$$
\begin{gathered}
\| m((u(t)+w(t)))^{m-1}-\left(\bar{u}(t)+w(t)^{m-1}\right) \\
\Delta_{g, T} w(t)\left\|_{E_{0}} \leq C\right\| u-\bar{u}\left\|_{E_{q}}\right\| \Delta_{g} w(t, \cdot) \|_{E_{0}} .
\end{gathered}
$$

Next, we define $h(t, u):=(u+w(t))^{m-2}|\nabla(u+w(t))|_{g}^{2}$

$$
\begin{aligned}
h(t, u)-h(t, \bar{u})= & (u+w(t))^{m-2}|\nabla(u+w(t))|_{g}^{2}-(\bar{u}+w(t))^{m-2}|\nabla(\bar{u}+w(t))|_{g}^{2} \\
= & \left((u+w(t))^{m-2}-(\bar{u}+w(t))^{m-2}\right)|\nabla(u+w(t))|_{g}^{2} \\
& +(\bar{u}+w(t))^{m-2}\left(|\nabla(u+w(t))|_{g}^{2}-|\nabla(\bar{u}+w(t))|_{g}^{2}\right) \\
= & \left((u+w(t))^{m-2}-(\bar{u}+w(t))^{m-2}\right)|\nabla(u+w(t))|_{g}^{2} \\
& +(\bar{u}+w(t))^{m-2}\langle\nabla(u-\bar{u}), \nabla(u+w(t))\rangle_{g} \\
& +(\bar{u}+w(t))^{m-2}\langle\nabla(\bar{u}+w(t)), \nabla(u-\bar{u})\rangle_{g} .
\end{aligned}
$$


For $v, \bar{v} \in E_{q}$ we can estimate the $L_{q}$ inner product of the gradient:

$$
\|\langle\nabla v, \nabla \bar{v}\rangle\|_{E_{0}} \leq C\|v\|_{C^{1}(X)}\|\bar{v}\|_{H_{p}^{1}(X)} \leq C\|v\|_{E_{q}}\|\bar{v}\|_{E_{q}}
$$

Therefore, for all $u, \bar{u} \in U$ and $t \in J$ the following estimates hold

$$
\begin{aligned}
|\nabla(u+w(t))|^{2} & \leq\|u+w(t)\|_{E_{q}}^{2} \leq C \\
|\langle\nabla(u-\bar{u}), \nabla(u+w(t))\rangle| & \leq\|u-\bar{u}\|_{E_{q}}\|u+w(t)\|_{E_{q}} \leq C\|u-\bar{u}\|_{E_{q}} \\
|\langle\nabla(\bar{u}+w(t), \nabla(u-\bar{u}))\rangle| & \leq\|\bar{u}+w(t)\|_{E_{q}}\|u-\bar{u}\|_{E_{q}} \leq C\|u-\bar{u}\|_{E_{q}} .
\end{aligned}
$$

In view of Eqs. (6.5) and (6.6), these estimates imply that for all $u, \bar{u} \in U$ and $t \in J$ the following estimate holds

$$
\|h(t, u)-h(t, \bar{u})\|_{E_{0}} \leq C\|u-\bar{u}\|_{E_{q}} .
$$

Thus, $F$ satisfies Assumption (F). We apply Theorem 6.1 to Problem (6.4), which proves Theorem 1.9.

Acknowledgements The results in this article are based on the second author's thesis, see [26]. His work was partly supported by Deutsche Forschungsgemeinschaft through the research training group GRK 1463. The authors thank K. Taira and Ch. Walker for helpful discussions and the referees for their suggestions that led to an improvement of the results.

Funding Open Access funding enabled and organized by Projekt DEAL.

Data availability Data sharing not applicable to this article as no datasets were generated or analyzed during the current study.

\section{Declarations}

Conflict of interest On behalf of all authors, the corresponding author states that there is no conflict of interest.

Open Access This article is licensed under a Creative Commons Attribution 4.0 International License, which permits use, sharing, adaptation, distribution and reproduction in any medium or format, as long as you give appropriate credit to the original author(s) and the source, provide a link to the Creative Commons licence, and indicate if changes were made. The images or other third party material in this article are included in the article's Creative Commons licence, unless indicated otherwise in a credit line to the material. If material is not included in the article's Creative Commons licence and your intended use is not permitted by statutory regulation or exceeds the permitted use, you will need to obtain permission directly from the copyright holder. To view a copy of this licence, visit http://creativecommons.org/licenses/by/4.0/.

\section{References}

1. Abels, H.: Pseudodifferential boundary value problems with non-smooth coefficients. Commun. Partial Differ. Equ. 30(10-12), 1463-1503 (2005)

2. Agmon, S.: On the eigenfunctions and on the eigenvalues of general elliptic boundary value problems. Commun. Pure Appl. Math. 15, 119-147 (1962) 
3. Amann, H.: Linear and quasilinear parabolic problems. In: Vol. I, Monographs in Mathematics, vol. 89 (Birkhäuser Boston, Inc., Boston, 1995) (Abstract linear theory)

4. Amann, H.: Linear and quasilinear parabolic problems. In: Vol. II, Monographs in Mathematics, vol. 106 (Birkhäuser/Springer, Cham), p. 2

5. Amann, H., Hieber, M., Simonett, G.: Bounded $H_{\infty}$-calculus for elliptic operators. Differ. Integral Equ. 7(3-4), 613-653 (1994)

6. Ammann, B., Große, N., Nistor, V.: Well-posedness of the Laplacian on manifolds with boundary and bounded geometry. Mathematische Nachrichten 292(6), 1213-1237 (2019)

7. Bergh, J., Löfström, J.: Interpolation spaces. An introduction. Springer, Berlin (1976). Grundlehren der Mathematischen Wissenschaften, No. 223

8. Boutet de Monvel, L.: Boundary problems for pseudo-differential operators. Acta Math. 126(1-2), 11-51 (1971)

9. Bilyj, O., Schrohe, E., Seiler, J.: $H_{\infty}$-calculus for hypoelliptic pseudodifferential operators. Proc. Am. Math. Soc. 138(5), 1645-1656 (2010)

10. Clément, P., Li, S.: Abstract parabolic quasilinear equations and application to a groundwater flow problem. Adv. Math. Sci. Appl. 3(Special Issue), 17-32 (1993/94)

11. Coriasco, S., Schrohe, E., Seiler, J.: Bounded $H_{\infty}$-calculus for differential operators on conic manifolds with boundary. Commun. Partial Differ. Equ. 32(1-3), 229-255 (2007)

12. Denk, R., Dore, G., Hieber, M., Prüss, J., Venni, A.: New thoughts on old results of R. T. Seeley. Math. Ann. 328(4), 545-583 (2004)

13. Denk, R., Hieber, M., Prüss, J.: $\mathcal{R}$-boundedness, Fourier multipliers and problems of elliptic and parabolic type. Mem. Am. Math. Soc. 166(788), 114 (2003)

14. Dines, N., Harutjunjan, G., Schulze, B.-W.: Mixed boundary value problems and parametrices in the edge calculus. Bull. Sci. Math. 131(4), 325-360 (2007)

15. Dore, G., Venni, A.: On the closedness of the sum of two closed operators. Math. Z. 196(2), 189-201 (1987)

16. Egorov, J.V., Kondrat'ev, V.A.: The oblique derivative problem. Mat. sb. (N.S.) 78(120), 148-176 (1969) (Russian)

17. Große, N., Schneider, C.: Sobolev spaces on Riemannian manifolds with bounded geometry: general coordinates and traces. Math. Nachr. 286(16), 1586-1613 (2013)

18. Grubb, G.: Pseudo-differential boundary problems in $L_{p}$ spaces. Commun. Partial Differ. Equ. 15(3), 289-340 (1990)

19. Grubb, G.: Functional Calculus of Pseudodifferential Boundary Problems, 2nd ed. Progress in Mathematics, vol. 65 (Birkhäuser Boston, Inc., Boston, 1996)

20. Grubb, G., Hörmander, L.: The transmission property. Math. Scand. 67(2), 273-289 (1990)

21. Grubb, G., Schrohe, E.: Trace expansions and the noncommutative residue for manifolds with boundary. J. Reine Angew. Math. 536, 167-207 (2001)

22. Hörmander, L.: Pseudo-differential operators and hypoelliptic equations, singular integrals. In: Proceedings of Symposia in Pure Mathematics, Vol. X, Chicago, Illinois, 1966, American Mathematical Society, Providence, RI, pp. 138-183 (1967)

23. Hörmander, L.: The boundary problems of physical geodesy. Arch. Rational Mech. Anal. 62(1), 1-52 (1976)

24. Kannai, Y.: Hypoellipticity of certain degenerate elliptic boundary value problems. Trans. Am. Math. Soc. 217, 311-328 (1976)

25. Krainer, T.: A calculus of abstract edge pseudodifferential operators of type $\rho, \delta$, Elliptic and parabolic equations. In: Escher, J., Schrohe, E., Seiler, J., Walker, C. (eds.) Springer Proceedings in Mathematics and Statistics, vol. 119. Springer, Cham, pp. 179-207 (2015)

26. Krietenstein, T.: Bounded $H^{\infty}$-calculus for a degenerate elliptic boundary value problem, Dissertation, Leibniz Universität Hannover (2019). https://doi.org/10.15488/8829

27. Kunstmann, P.C., Weis, L.: Maximal $L_{p}$-Regularity for Parabolic Equations, Fourier Multiplier Theorems and $H^{\infty}$-Functional Calculus, Functional Analytic Methods for Evolution Equations, Lecture Notes in Mathematics, pp. 65-311. Springer, Berlin (1855)

28. Kumano-go, H.: Pseudodifferential Operators. MIT Press, Cambridge (1981). (Translated from the Japanese by the author, Rémi Vaillancourt and Michihiro Nagase)

29. McIntosh, A.: Operators which have an $H_{\infty}$ functional calculus, miniconference on operator theory and partial differential equations (North Ryde, 1986). In: Proceedings of the Centre for Mathematical 
Analysis, Australian National University, vol. 14, Australian Naional University, Canberra (1986), pp. 210-231

30. Michael, E.: Continuous selections. I. Ann. Math. (2) 63, 361-382 (1956)

31. Prüss, J.: Maximal Regularity for Evolution Equations in $L_{p}$-Spaces (2002). Lecture notes for the Summer School 'Positivity and Semigroups', Monopoli, September (2002). https://www2.mathematik. uni-halle.de/reports/sources/2002/02-24report.ps. Accessed 9 Aug 2021

32. Rempel, S., Schulze, B.-W.: Index Theory of Elliptic Boundary Problems. North Oxford Academic Publishing Co. Ltd., London (1985). (Reprint of the 1982 edition)

33. Roidos, Nikolaos, Schrohe, Elmar: Existence and maximal $L^{p}$-regularity of solutions for the porous medium equation on manifolds with conical singularities. Commun. Partial Differ. Equ. 41(9), 14411471 (2016)

34. Schick, T.: Manifolds with boundary and of bounded geometry. Math. Nachr. 223, 103-120 (2001)

35. Schrohe, E.: A Short Introduction to Boutet de Monvel's Calculus, Approaches to Singular Analysis (Berlin, 1999), Operator Theory: Advances and Applications, pp. 85-116. Birkhäuser, Basel (2001)

36. Schulze, B.-W.: Pseudo-differential Operators on Manifolds with Singularities, Studies in Mathematics and Its Applications, vol. 24. North-Holland Publishing Co., Amsterdam (1991)

37. Seeley, R.: Norms and domains of the complex powers $A_{B} z$. Am. J. Math. 93, 299-309 (1971)

38. Seeley, R.: Trace expansions for the Zaremba problem. Commun. Partial Differ. Equ. 27(11-12), 2403-2421 (2002)

39. Sogge, C.D.: Fourier Integrals in Classical Analysis, 2nd ed. Cambridge Tracts in Mathematics. Cambridge University Press, Cambridge (2017)

40. Strichartz, R.S.: Analysis of the Laplacian on the complete Riemannian manifold. J. Funct. Anal. 52(1), 48-79 (1983)

41. Taira, K.: Boundary Value Problems and Markov Processes, 3rd ed. Lecture Notes in Mathematics. Springer Nature, Cham (2020)

42. Taira, K.: On some degenerate oblique derivative problems. J. Fac. Sci. Univ. Tokyo Sect. IA Math. 23(2), 259-287 (1976)

43. Taira, K.: Analytic Semigroups and Semilinear Initial Boundary Value Problems, 2nd ed. London Mathematical Society Lecture Note Series. Cambridge University Press, Cambridge (2016)

44. Taira, K.: Semigroups, Boundary Value Problems and Markov Processes. Springer Monographs in Mathematics, 2nd edn. Springer, Heidelberg (2014)

45. Taira, K.: Bifurcation curves in a combustion problem with general Arrhenius reaction-rate laws. Ann. Univ. Ferrara Sez. VII Sci. Mat. 62(2), 337-371 (2016)

46. Taylor, M.: Pseudodifferential Operators and Nonlinear PDE, Progress in Mathematics. Birkhäuser, Basel (1991)

47. Triebel, H.: Interpolation Theory, Function Spaces, Differential Operators, North-Holland Mathematical Library, vol. 18. North-Holland Publishing Co., Amsterdam (1978)

48. Triebel, H.: Theory of Function Spaces. II, Monographs in Mathematics. Birkhäuser Verlag, Basel (1992)

Publisher's Note Springer Nature remains neutral with regard to jurisdictional claims in published maps and institutional affiliations. 